University of Louisville

ThinkIR: The University of Louisville's Institutional Repository

Electronic Theses and Dissertations

$5-2021$

\title{
Simulation of fracture strength improvements of a human proximal femur using finite element analysis.
}

Waleed Ebraheem Alim

University of Louisville

Follow this and additional works at: https://ir.library.louisville.edu/etd

Part of the Biomechanical Engineering Commons, and the Other Biomedical Engineering and Bioengineering Commons

\section{Recommended Citation}

Alim, Waleed Ebraheem, "Simulation of fracture strength improvements of a human proximal femur using finite element analysis." (2021). Electronic Theses and Dissertations. Paper 3591.

https://doi.org/10.18297/etd/3591

This Doctoral Dissertation is brought to you for free and open access by ThinkIR: The University of Louisville's Institutional Repository. It has been accepted for inclusion in Electronic Theses and Dissertations by an authorized administrator of ThinkIR: The University of Louisville's Institutional Repository. This title appears here courtesy of the author, who has retained all other copyrights. For more information, please contact thinkir@louisville.edu. 


\title{
SIMULATION OF FRACTURE STRENGTH IMPROVEMENTS OF A HUMAN PROXIMAL FEMUR USING FINITE ELEMENT ANALYSIS
}

\author{
By \\ Waleed Ebraheem Alim
}

\begin{abstract}
A Dissertation
Submitted to the Faculty of the

J. B. Speed School of Engineering of the University of Louisville

In Partial Fulfillment of the Requirements

for the Degree of
\end{abstract}

Doctor of Philosophy in Mechanical Engineering

Mechanical Engineering Department

Orthopaedic Bioengineering Laboratory

University of Louisville

Louisville, Kentucky

May 2021 

SIMULATION OF FRACTURE STRENGTH IMPROVEMENTS OF A HUMAN PROXIMAL FEMUR USING FINITE ELEMENT ANALYSIS

\author{
By \\ Waleed Ebraheem Alim
}

A Dissertation Approved on 2/5/2021

By the following Dissertation Committee

\begin{tabular}{c} 
Dissertation Chair \\
Michael J. Voor, $\mathrm{PhD}$ \\
\hline Roger D. Bradshaw, $\mathrm{PhD}$ \\
\hline
\end{tabular}

Thomas A. Berfield, PhD

Ayman S. El-Baz, PhD 


\section{ACKNOWLEDGEMENTS}

I would like to thank Michael J. Voor, $\mathrm{PhD}$, my advisor, for providing me invaluable guidance and advice on my academic and professional career. For always showing me another way to approach the challenges of research, get to a solution, and answer the questions that need answered. I want to thank my committee members Robert Bradshaw, PhD, Thomas A Berfield, PhD, and Ayman S Elbaz, PhD for their council and support to finish. I would like to thank Gina Bertocci, PhD and her team Angela $\mathrm{K}$ Thompson, PhD, Raymond Dsouza, PhD, Nathan Brown, PhD, and Ms. Keyonna Mckinsey for supporting me using Mimics/3-Matic software in their computer lab. I would especially like to thank Ayman S Elbaz, $\mathrm{PhD}$ and his professional associate Ahmed Soliman, PhD, Ahmed Magdy, PhD, Mohammed Elmogy, PhD, and Ahmed Shaffie, PhD for assisting me in image processing and using their computer lab. I would like to thank my special colleague Jeffery $\mathrm{W}$ Borden, $\mathrm{PhD}$ who supported me through various topics in my project. I would like to thank the many colleagues who supported me through the many trials and tribulations of obtaining a doctorate, Mr. Seid W. Waddell, Jr., Harrison Simrall, and Diane Jenne. Each of them contributing in their own way to my success. I would also like to thank the Mechanical Engineering Department staff and faculty. I would especially like to thank Kevin Murphy, $\mathrm{PhD}$, for the behind-the-scene support to make this pursuit possible. 
Most importantly, a deep, heartfelt appreciation and dedication goes to my loving parents who have been praying for me and encouraging me to achieve my doctorate. To my fathers and mothers in law who supported me financially and socially. To my wife, Maram Kateb, for her unwavering love and support during the pursuit of this dream. I will always be grateful for all her sacrifice. To my children, Mawadah, Massarah, Mohammed, and Abdulaziz, I would like to thank them for the sacrifices they are yet aware of that have aided me in completing the doctorate. I look forward to spending a whole lot more time enjoying them. To my sisters and brothers Wisam, Esraa, Ahlam, Dr. Sarah, and Dr. Abdullah who have been encouraging me to succeed. To my brothers in law, Mohammed Alhairi, Haitham Faidah, Amjad Kateb, Ahmed Kateb, and Anwar Kateb. Finally, my best friends Bandar Alquthamy, Wael Alsayed, Subhi Alghamdi, and Khaled Ibrahim Ahmed, $\mathrm{PhD}$ who supported me in many areas that directly or indirectly relevant to my project 


\section{ABSTRACT \\ SIMULATION OF FRACTURE STRENGTH IMPROVEMENTS OF A HUMAN PROXIMAL FEMUR USING FINITE ELEMENT ANALYSIS}

\section{WALEED ALIM}

$2 / 5 / 2021$

The most common hip fracture in the elderly occurs as a result of a fall to the side with impact over the greater trochanter resulting in a fracture of the proximal femur. The fracture usually involves the femoral neck or the intertrochanteric region. It has recently been determined that the fracture crack of a hip fracture typically initiates on the superiorlateral cortex of the femoral neck and then propagates across the femoral neck, resulting in a complete fracture. The strength of the superior-lateral cortex of the femoral neck is likely determined by the combined properties of the generally thin cortex (outer layer) and the underlying trabecular bone in this region. The objective of this study was to determine the relative effects of increasing or decreasing the thickness of these bone tissues on the overall failure strength of the proximal femur. The clinical significance of this work relates to hip fracture risk with various potential treatment options to improve either cortical or trabecular bone quality. 
A human femur obtained from a 68 year old female donor was scanned using computed tomography at 60-micron voxel resolution and a series of high-resolution finite element models were generated. The models were constructed with a base-element dimension of 120 microns and models included a basic model with cortical and trabecular thicknesses representative of the cadaver specimen from the original scan. Other models used a standardized algorithm to either dilate or erode the trabecular and cortical bone compartments of the femoral neck so that a total of nine models were created including the basic model. Each model was used to simulate a fall-to-the-side loading condition with appropriate boundary and loading conditions as used in previous models and experiments. An experimental test of the cadaver femur was also performed with three strain gauges placed on the proximal femur: on the superior-lateral cortex, on the inferior-medial cortex, and on the medial cortex positioned distal to the lesser trochanter. This femur was loaded at a rate of $100 \mathrm{~mm} / \mathrm{s}$ until fracture of the femoral neck using a standard fall-to-the-side setup and the applied load and gauge strains were recorded. The femur neck fractured at a load of $2140 \mathrm{~N}$. To validate the basic finite element model, the strain gauge strains at the load levels of $1000 \mathrm{~N}$ and $2000 \mathrm{~N}$ were compared to the calculated strains from the basic model at the same loads and same location as the gauge on the cadaver femur. After the basic model was validated, a failure criterion was determined as the volume percentage of the elements in the model that had exceeded $7000 \mu \varepsilon$ at the failure load corresponding to the load at which the cadaver femur failed. Subsequently, this failure criterion was applied to the other eight models as a parametric analysis to estimate the increase or decrease in failure strength caused by the changes in cortical and trabecular thickness. 
The validation test results showed that the basic finite element model calculated strain on the superolateral cortex was within $2.1 \%$ of the experimentally measured strain at $1000 \mathrm{~N}$ loading. The validated basic model was then used to determine that the percentage of finite elements (by volume of the model) in excess of $7000 \mu \varepsilon$ at the failure load was $4.2 \%$. This failure criterion was then used to estimate the failure load for the other eight models with different combinations of either thicker $(+120 \mu \mathrm{m})$ or thinner $(-120 \mu \mathrm{m})$ cortex and trabeculae in the femoral neck. The calculated failure loads ranged from $324 \mathrm{~N}$ for the model with thinned cortex and thinned trabeculae to $3336 \mathrm{~N}$ for the model with thickened cortex and thickened trabeculae. The model with normal cortex and thickened trabeculae had a failure load of $3242 \mathrm{~N}$, which is only $2.8 \%$ less than the strongest case.

The largest single parameter effect on proximal femoral strength is realized by an increase in trabecular thickness. This is somewhat surprising considering that cortical bone is typically stronger than cancellous bone. However, the spatial arrangement of trabecular bone and the buttress support it provides to the thin cortex apparently plays an important role in the ability of a global increase in thickness to have a significant beneficial effect. 


\section{TABLE OF CONTENTS}

ACKNOWLEDGEMENTS ............................................................................................ iii

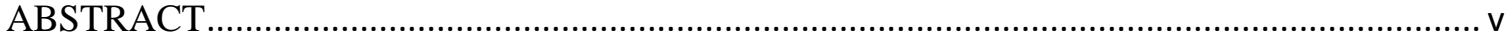

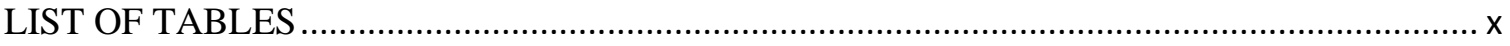

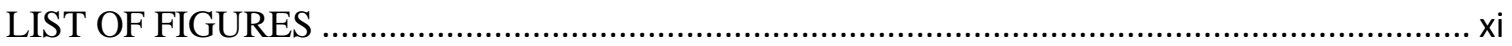

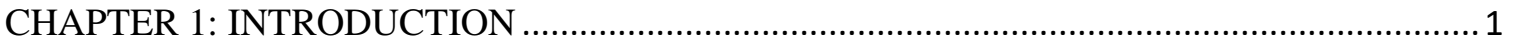

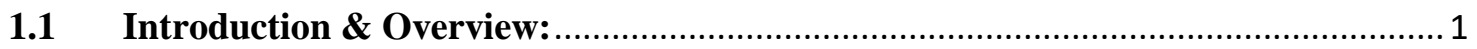

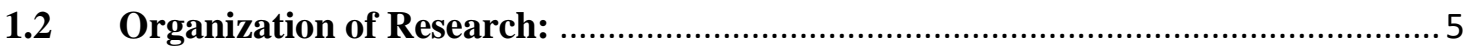

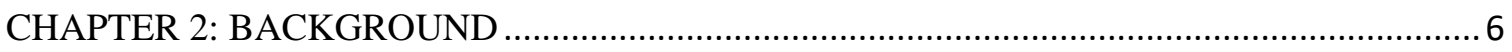

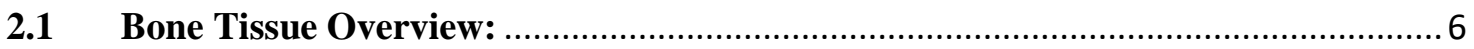

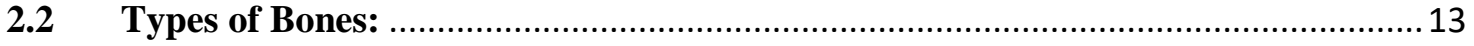

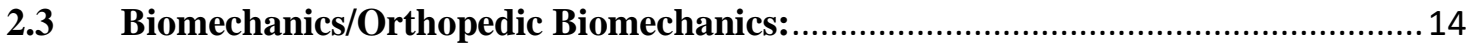

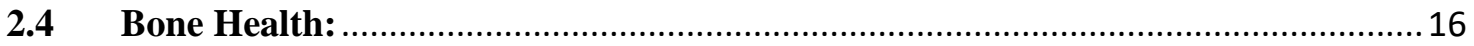

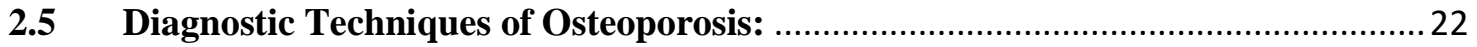

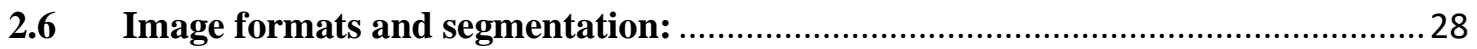

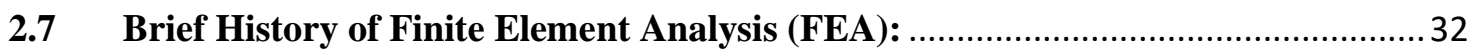

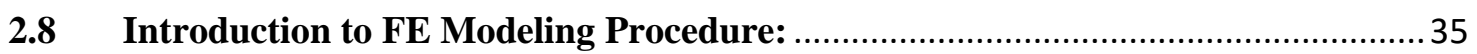

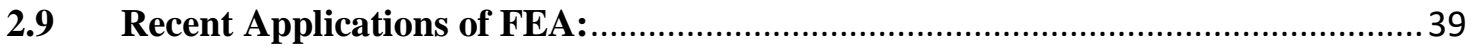

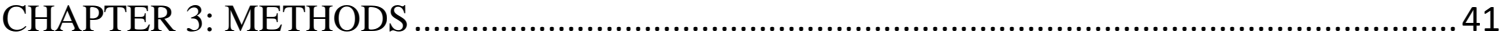

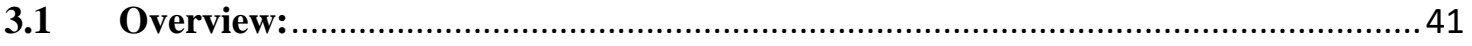

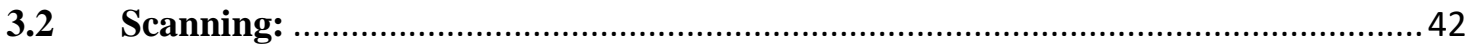

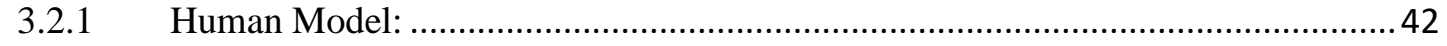

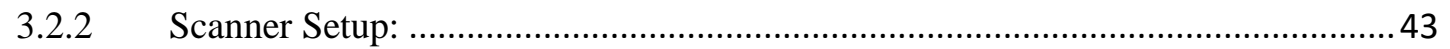

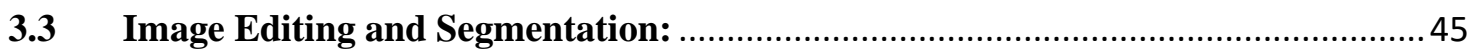

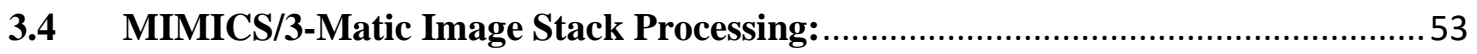

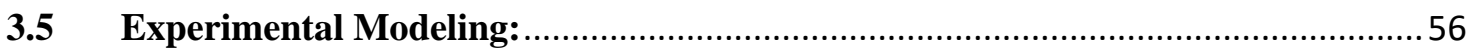

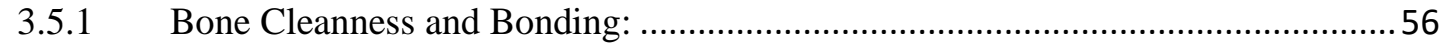

3.5.2 Strain Gauges Installation and Experiment Setup:...............................................57 
3.5.3 Loading and Boundary Conditions: .............................................................. 57

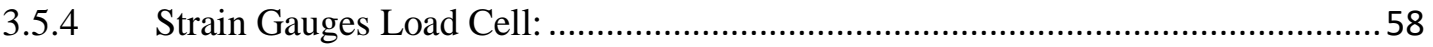

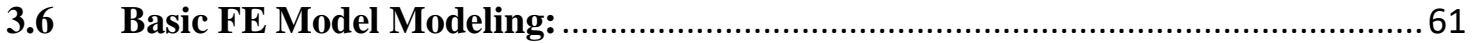

3.6.1 Basic FE Model Execution Method: …….............................................................. 61

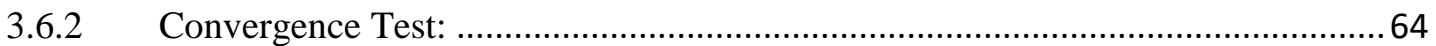

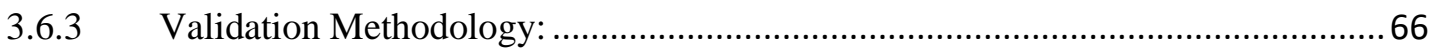

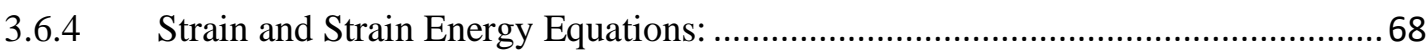

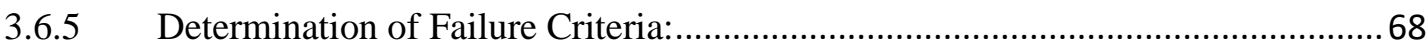

3.7 FE Analysis for Altered Parameters: ............................................................... 70

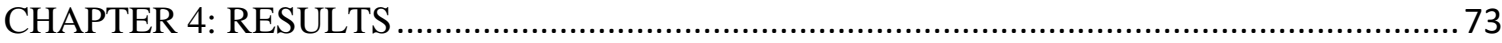

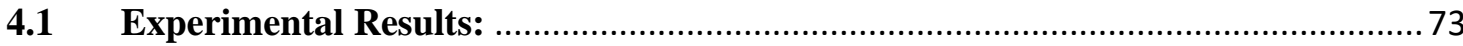

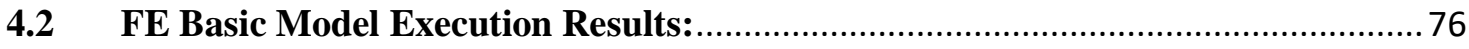

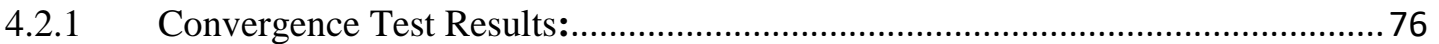

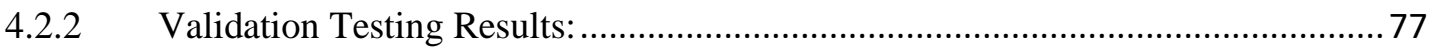

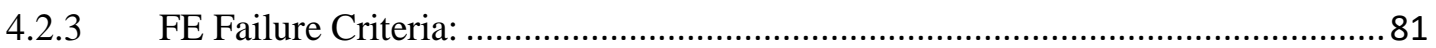

4.3 Failure Loads for FE Models with Altered Parameters: .....................................83

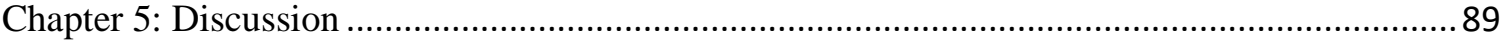

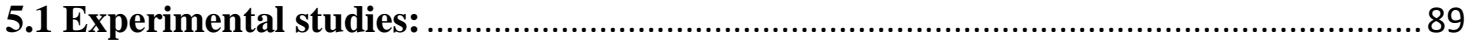

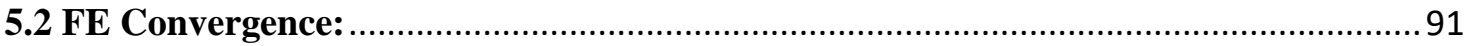

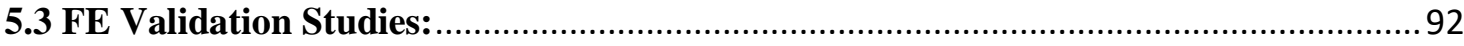

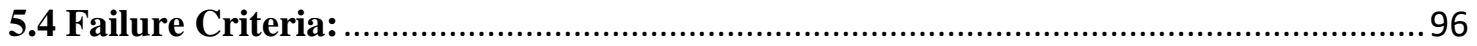

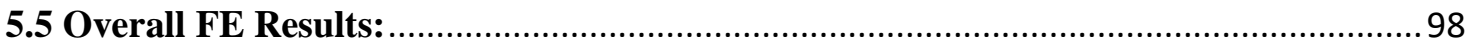

5.6 The Importance of Interaction and Relative Role of the Trabeculae Versus Cortical

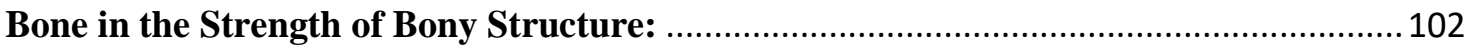

5.7 Hip Fracture Prevention Procedure: ..................................................................... 104

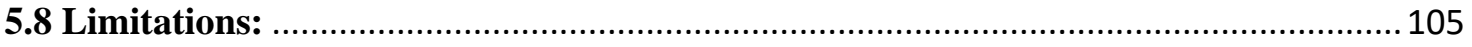

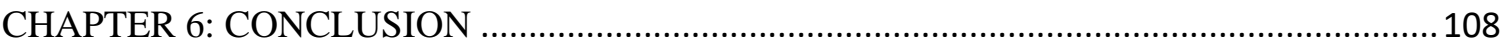

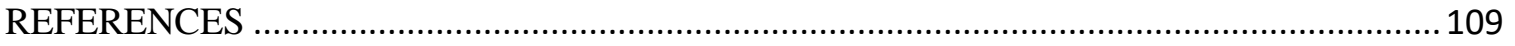

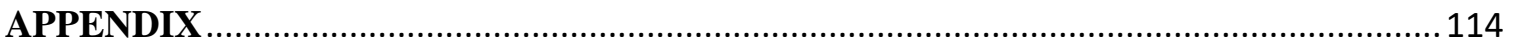

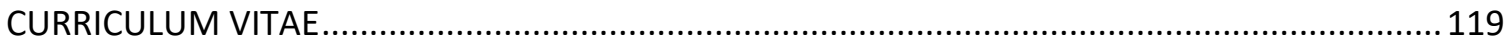




\section{LIST OF TABLES}

Table 1: As the time advances, the force and strains increase until the first peak occurred at the force $-2140 \mathrm{~N}$ 74

Table 2: Values reported from ABAQUS into Excel for strain gauge \#3. The variables E11, E33, E13, and $\varepsilon x^{\prime}$ represent normal and shear strains and resultant strain as the strain gauge angle was included in calculations. .................................................................... 79 Table 3: Values reported from ABAQUS into Excel for strain gauge \#4. The variables E11, E33, E13, and $\varepsilon x$ ' represent normal and shear strains and resultant strain as the strain gauge angle was included in calculations

Table 4: Excel calculations revealed well agreement at $1000 \mathrm{~N}$ between the calculated and measured strains for the strain gauge 3 , but the calculated value for the gauge located at the inferior neck (strain4) differed more than 10\% 81

Table 5: The calculated effective strain at the superior neck differed from the measured one by less than $10 \%$ 81

Table 6: The volume fraction of failed tissue was calculated by dividing the failed volume over the femur volume 83

Table 6: The failure load estimated at $4.2 \%$ using linear interpolation method for dilated both cortical and trabecular bone 84

Table 7: The failure load estimated at $4.2 \%$ using linear interpolation method for normal cortical and dilated trabecular bone..... 84

Table 8: The failure load estimated at $4.2 \%$ using linear interpolation method for eroded cortical and dilated trabecular bone 85 Table 9: The failure load estimated at $4.2 \%$ using linear interpolation method for dilated cortical and normal trabecular bone. 85 Table 10: The failure load estimated at $4.2 \%$ using linear interpolation method for eroded cortical and normal trabecular bone. 86 Table 11: The failure load estimated at $4.2 \%$ using linear interpolation method for dilated cortical and eroded trabecular bone..... 86 Table 12: The failure load estimated at $4.2 \%$ using linear interpolation method for normal cortical and eroded trabecular bone.....

Table 13: The failure load estimated at $4.2 \%$ using linear interpolation method for eroded cortical and eroded trabecular bone..... 87

Table 14: FE Models Ranking Based on Failure Loads as the differences between loads and volumes were calculated 


\section{LIST OF FIGURES}

Figure 1: Anterior (left) and posterior (right) proximal femur anatomy [20] ................. 10

Figure 2: Femoral bone components [21] ................................................................. 11

Figure 3: Healthy bone (left) vs. Osteoporotic bone (right) [22] .................................. 11

Figure 4: Maximal principal strain in the selected plane though the femoral neck in the healthy (A) and osteoporotic (B) femur [22] .............................................. 12

Figure 5: Typical apparent level load-displacement curve of biomechanical behavior

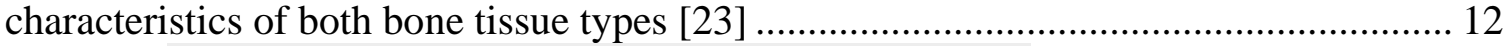

Figure 6: Women lose bone mass more rapidly than men [30] .................................. 16

Figure 7: Distribution of bone mineral density in healthy women aged 30-40 years [9]. 18

Figure 8: Remaining lifetime risk of hip fracture in women aged 50 years, according to bone mineral density (BMD) or T score at the hip [9] ................................... 18

Figure 9: Distribution of bone mineral density in women of different ages, and the

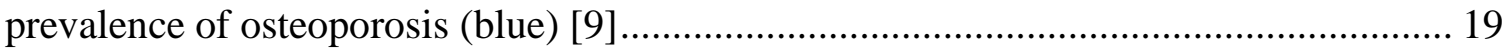

Figure 10: ACTIS HRCT laboratory scanner used in the current study .......................... 26

Figure 11: Key ACTIS system components [50] ....................................................... 26

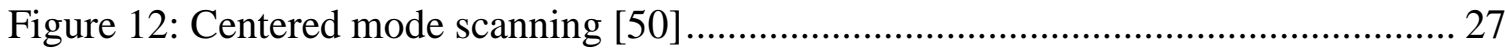

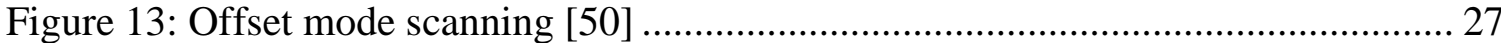

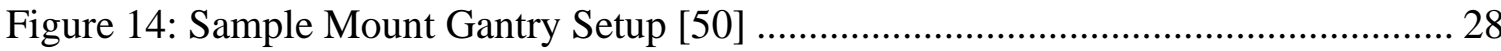

Figure 15: Stack of 2D images being converted to a 3D surface in MIMICS program [53]

Figure 16: Main window overview of 3-matic program. Featured are the menu toolbar (1), 3D view (2), object tree (3), properties (4), and the logger (5) [54] ......................... 30

Figure 17: Discretization a circle from 4 sides to 8 sides polygon ................................ 34

Figure 18: 3D FE domains of tetrahedral and hexahedral elements [58] ....................... 36

Figure 19: Hexahedral element (left); Tetrahedral element (right) [60] .......................... 36

Figure 20: A microscopic computed tomography (Micro-CT) scanner [65] .................. 43

Figure 21: The original gray image (left) and the cropped image (right) in ImageJ ....... 45

Figure 22: The original gray image (left) and the brighter gray image (right) in ImageJ 46

Figure 23: Double selection was used in MIPAV for images which has two separate

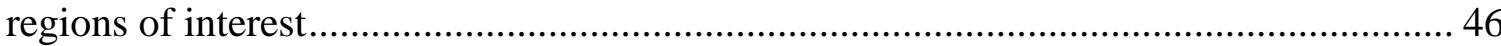

Figure 24: The mask image (middle) was created from the original image (left). The right image is the product of the left image and the middle image in ImageJ

Figure 25: The original image (a), A created mask for the cancellous (b), A separated

cancellous image (c), and the separated cortical bone (d) in ImageJ.............................. 48

Figure 26: A brightness and contrast performed on the left image to get the right image in Image J 
Figure 27: The blurred regions were removed from the left image in ImageJ to obtain the

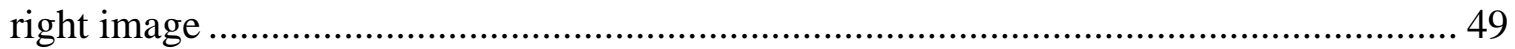
Figure 28: An automatic threshold was applied over the left image in ImageJ to obtain the right image.... 49 Figure 29: The unconnected regions were removed from the left image to get the new

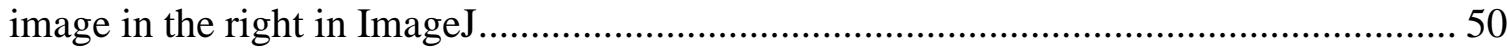
Figure 30: The original gray image of cancellous on the left converted to binary image on

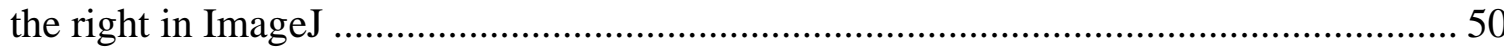
Figure 31: The original gray image of cortical bone on the left converted to binary image

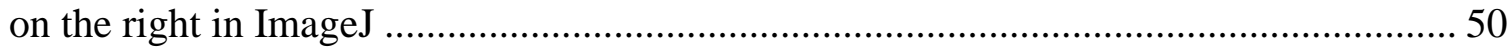
Figure 32: The combination between the binary images for cancellous and trabecular

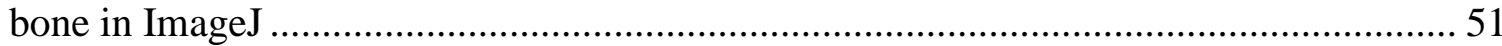
Figure 33: Binary options in ImageJ for erosion or dilation processes. "Iterations" specify number of times for a process and "Counts" specifies number of adjacent pixels in background necessary before a process ............................................................... 52 Figure 34: The binary image for the basic model in the middle was 3-dimentionally

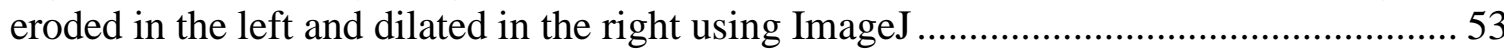
Figure 35: The left image from ImageJ with $60 \mu \mathrm{m}$ was imported into Mimics, from which a new binary mask was generated as illustrated in the green image using Mimics 54 Figure 36: Region growing tool in MIMICS to create a new mask for connected regions

Figure 37: A region growing operation was performed over the left image to get the right image in Mimics. As noticed in the right image where the unconnected region with the green color was excluded from the new mask with the yellow color [52] ..................... 55 Figure 38: The calculated 3D surface part in Mimics was transferred into 3-Matic for re-

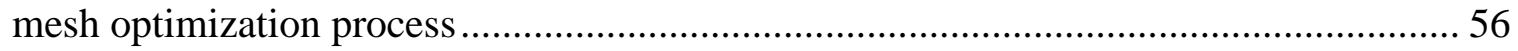
Figure 39: The 3D surface meshing completed in 3-Matic ......................................... 56 Figure 40: The cadaveric femur embedded in a plastic pipe with a Bondo body filler .... 57 Figure 41: A strain gauge was placed on the superior neck of the cadaveric femur on the left. The other two strain gauges were placed on the inferior neck and the medial shaft. 57 Figure 42: The setting of the biomechanical testing for the cadaveric proximal femur

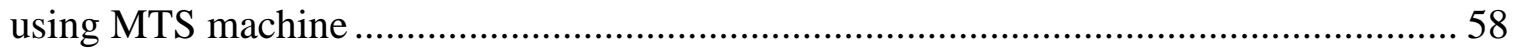

Figure 43: The Wheatstone half bridge circuit [66]................................................. 59 Figure 44: Exemplary force-displacement curve for a proximal femur fracture behavior. The yield region indicates to the fracture initiated, and the cross sign indicates to full

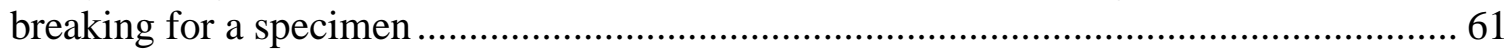

Figure 45: The loading condition applied on the femur head in ABAQUS .................... 63 Figure 46: Boundary conditions applied on the trochanter and the bottom in ABAQUS 63 Figure 47: The left binary image in ImageJ, whereas the right image in ABAQUS.

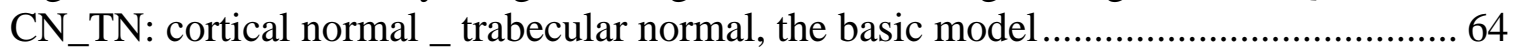
Figure 48: The red colored node inside a little circle chosen for convergence test in ABAQUS

Figure 49: The 3D triangular surface in the left was converted to a 3D solid tetrahedral part in the right with 4-noded element mesh in ABAQUS ...................................... 65

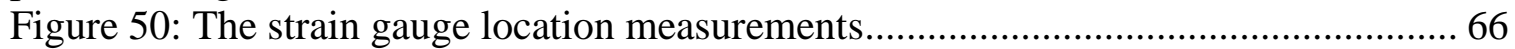

Figure 51: The strain gauge location determination in ABAQUS ................................. 67 
Figure 52: The strain gauge angle measured virtually on the screen 67

Figure 53: The green area represents the strain gauge location. The red highlighted elements were selected for calculations and analysis .....

Figure 54: The expected resulted stresses distribution due to sideways falling [68]........ 69

Figure 55: The left binary image in ImageJ, whereas the right image in ABAQUS.

CD_TN: cortical dilated _ trabecular normal ........................................................... 70

Figure 56: The left binary image in ImageJ, whereas the right image in ABAQUS.

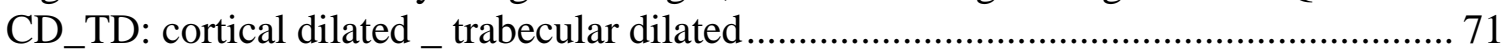

Figure 57: The left binary image in ImageJ, whereas the right image in ABAQUS.

CD_TE: cortical dilated _trabecular eroded .............................................................. 71

Figure 58: The left binary image in ImageJ, whereas the right image in ABAQUS.

CN_TD: cortical normal _ trabecular dilated .............................................................. 71

Figure 59: The left binary image in ImageJ, whereas the right image in ABAQUS.

CN_TE: cortical normal _ trabecular eroded.................................................................. 71

Figure 60: The left binary image in ImageJ, whereas the right image in ABAQUS.

CE_TN: cortical eroded _ trabecular normal .............................................................. 72

Figure 61: The left binary image in ImageJ, whereas the right image in ABAQUS.

CE_TE: cortical eroded _ trabecular eroded................................................................. 72

Figure 62: The left binary image in ImageJ, whereas the right image in ABAQUS.

CE_TD: cortical eroded _trabecular dilated ................................................................. 72

Figure 63: A human proximal femur subjected to a sideways fall experimental test. The curve illustrates the femur behavior represented by force(N) vs. time(sec)................... 74 Figure 64: The chart illustrates the experimental data of time vs force and 3 strains. The arrows point to strain values reported from strain gauge \#3 at both loading increments of $1000 \mathrm{~N}$ and $2000 \mathrm{~N}$

Figure 65: Experimental results revealed linear relationships between values of each strain gauge and corresponding values of force and other strain gauges. 75

Figure 66: The Femur when it was completely broken. The red arrows indicate the location of gauge 3 before and after breakage, whereas the blue arrow points to the probable failure initiation

Figure 67: The chart represents displacement vs. model degrees of freedom for both models, 4-noded element mesh (4,413,027 DOF) and 10-noded element mesh $(26,787,105 \mathrm{DOF})$ at the first loading increment $(1000 \mathrm{~N})$ and the second loading increment $(2000 \mathrm{~N})$ 77

Figure 68: The resulted strain contour where the maximum compressive strain regions shown with a black color, whereas the maximum tension strain shown with a gray color. The black arrow points to the location of the strain gauge \#3 ......................................... 78 Figure 69: The group of elements selected from the red highlighted region in the previous figure, which represents the location of the strain gauge \#3.......................................... 78

Figure 70: Frontal section of SED plot for the basic FE model at 2000N ....................... 82 Figure 71: SED plot displays a group of elements strained at 0.245 or higher at $2000 \mathrm{~N}$ for the basic model 83

Figure 72: SED plotted at 4000N for dilated cortical and dilated trabecular bone (left).

SED plot for a group of elements failed in excess of 0.245 (right) ................................. 84 Figure 73: SED plotted at 4000N for normal cortical and dilated trabecular bone (left). SED plot for a group of elements failed in excess of 0.245 (right) ................................. 84 
Figure 74: SED plotted at 4000N for eroded cortical and dilated trabecular bone (left). SED plot for a group of elements failed in excess of 0.245 (right)

Figure 75: SED plotted at 2000N for dilated cortical and normal trabecular bone (left). SED plot for a group of elements failed in excess of 0.245 (right)

Figure 76: SED plotted at 2000N for eroded cortical and normal trabecular bone (left).

SED plot for a group of elements failed in excess of 0.245 (right)

Figure 77: SED plotted at 1000N for dilated cortical and eroded trabecular bone (left). SED plot for a group of elements failed in excess of 0.245 (right)

Figure 78: SED plotted at $1000 \mathrm{~N}$ for normal cortical and eroded trabecular bone (left).

SED plot for a group of elements failed in excess of 0.245 (right)

Figure 79: SED plotted at $1000 \mathrm{~N}$ for eroded cortical and eroded trabecular bone (left).

SED plot for a group of elements failed in excess of 0.245 (right)

Figure 80: Strength vs volumetric bone mineral density (vBMD) for the current study (blue) and the study of the 12 femurs (red). The trend line of each study is illustrated . 100 


\section{CHAPTER 1: INTRODUCTION}

\subsection{Introduction \& Overview:}

Hip fractures are associated with high morbidity and mortality. Survivors of hip fractures suffer from loss of mobility and independence [1-5]. Hip fractures also represent one of the highest incidences as well as one of the highest levels of medical expenses compared to other types of fractures at other anatomical sites [1-5]. In the United States, the annual medical costs of osteoporotic fractures alone were expected to range between $\$ 16$ and \$25 billion dollars per year over the years from 2002 to 2025 [2, 5-7]. According to data from American Academy of Orthopedic Surgeons (AAOS), more than 340,000 Americans are hospitalized annually for hip fractures [3], and 12 to 33 percent of patients with hip fractures die within one year of their injury $[3,4,8,9]$. The Health Care Financing Administration reported in 1990 that the number of hip fracture cases for women is almost four times the number of cases for men. But even though more women suffer hip fractures than men, the problem is significant in the male population due to the large overall number of fractures. There are roughly 10 million Americans over age 50 with osteoporosis and an additional 34 million with osteopenia $[2,10]$. Thus, the "at risk" population is large.

Most hip fractures in the elderly occur as a result of a fall to the side with impact over the greater trochanter resulting in a fracture of the proximal femur. In fact, falls account for over $90 \%$ of all hip fractures in the elderly $[11,12]$. The fracture usually involves the femoral neck or the intertrochanteric region. It has recently been determined that the fracture crack of a typical hip fracture initiates on the superolateral cortex of the 
femoral neck and then propagates across the femoral neck, resulting in a complete fracture $[11,13]$. This underscores the importance of the mechanical behavior of the superolateral side of the femoral neck under fall-to-the-side loading. It is also known that most falls, even in the elderly, do not result in a hip fracture [13]. So, the conditions for a hip fracture from a fall must be very specific. Therefore, it is important to determine the most critical factors that are present when a fall does result in a fracture [11, 13].

Bone mineral density (BMD) is often used as a measure of bone quality and as a determinant for fracture risk. BMD quantifies the amount of bone tissue in a given volumetric space within the skeleton. To assess hip fracture risk, doctors measure BMD at the hip (proximal femur) using Dual-Energy-X-ray-Absorptiometry (DXA), a relatively safe, inexpensive, and reliable method that uses plane x-rays and compares the BMD status of a given patient to the population at large through a large database. The assessment status is classified as osteoporotic if the measured T-score is -2.5 standard deviations from the average value of normal healthy people. Doctors usually prescribe some treatment such as calcium or vitamin D to people with osteoporosis to increase BMD and to decrease the incidence of future fracture [14]. Compared to the risk for untreated postmenopausal women, treatments resulting in slight increases in BMD may reduce the risk of hip fracture by $50 \%$ [14]. Physical activities or specifically multicomponent exercise programs that include resistance training in combination with balance training are also recommended for people with osteoporosis [15]. People with osteoporosis are advised to not engage in aerobic training to the exclusion of resistance training or balance training [15]. Some doctors have recommended combination or sequential treatments of low dose hormone replacement therapy (HRT) and antiresorptive bisphosphonate drugs to reduce bone 
resorption more than using low doses of HRT alone [16].

Although medications do increase the bone fracture strength, they typically don't return bone strength back to a normal condition. Antiresorptive medications increase bone density by shutting down or slowing down the natural resorptive process, so the bone strength is eventually enhanced. However, the quality of the bone structure is not necessarily returned to what it was at a young adult age.

In addition to bone tissue quantity alone, bone architecture also plays an important role in bone strength. The structural arrangement of the components that make up a particular bone such as the proximal femur is just as important, if not more important, as BMD at determining the risk of fracture during a fall to the side [16]. Furthermore, the prediction of bone fracture risk also depends on both the boundary conditions and the loading conditions including loading-direction and anatomical sites of direct application of forces as well. This effect is made apparent by the low percentage of falls that actually result in a hip fracture. In other words, the conditions of the fall must be "just right" for the fracture to occur. Nevertheless, hundreds of thousands of hip fractures do occur each year in the US, and all of these factors must be considered.

The femur bone is highly complicated due to its great heterogeneity and irregularity of its geometry. Because of those properties, researchers have expanded their interests and concluded that the proximal femur is more efficient to be studied as a whole upon quality basis jointly with quantity basis. The strength of the femoral neck is likely determined by the combined properties of the generally thin cortex (outer layer) and the underlying trabecular bone in this region. According to the geometrical structure, the proximal femur has a higher stiffness and strength in the longitudinal direction than it does in the lateral 
direction. Therefore, the proximal femur is more susceptible to fracture when loaded in the lateral direction, such as from a fall.

High-resolution computed tomography (HRCT) scanning has shown reliable measurements for bone quantitative and qualitative parameters relevant to bone strength [16]. Furthermore, an exceptionally promising approach for bone strength prediction combines HRCT with Finite Element Analysis (FEA) and with supercomputer hardware to calculate complete stress and strain distributions in the bone tissue with great detail. Finite element analysis (FEA) based on CT datasets is now widely used in research and is being explored for clinical use to estimate patient specific bone strength as well as bone fracture risk.

The clinical significance of this work relates to hip fracture risk modification with various potential treatment options to improve either cortical or trabecular bone quality. In other words, would increasing the thickness of the trabeculae in the proximal femur without increasing the thickness of the cortex as well have a meaningful effect on improving fracture strength, or, vice-versa? Therefore, with the knowledge gained from these models, physicians may be able to target specific regions of the proximal femur for bone quality improvement through systemic or local application of pharmaceutical interventions or direct application of biologic products to stimulate bone growth.

\section{Aim:}

The objective of this study was to determine the relative effect of increasing or decreasing the thickness of the bone tissue in the femoral neck on the overall failure strength of the proximal femur subjected to loading due to falling to the side. Specifically, a valid fall-to-the-side proximal femur model was established, and the trabecular and 
cortical bone of the femoral neck were separately made thicker or thinner in a parametric analysis.

\subsection{Organization of Research:}

This research exploration encompasses experimental testing outcomes as well as the development and analysis of structural FE models. In Chapter 1 the goal and significance of the research is presented with the specific tasks to achieve the desired outcomes. Chapter 2 presents the background with the detailed coverage of bone structure and its failure mechanisms. Chapter 3 covers the descriptions of experimental method and the generation of accurate load-displacement FE models including mesh generation techniques. In Chapter 4, the results are presented for the experimental test, and FE

modeling. In Chapter 5, the results are discussed along with their relevance to the field of bone FE modeling and hip fracture research. 


\section{CHAPTER 2: BACKGROUND}

\subsection{Bone Tissue Overview:}

The skeletal system is the framework for the body's shape and the fundamental structure that enables the body to move normally [17]. It also has various functions that enable organisms to grow larger, move better, protect effectively, and heal faster. The skeletal system is comprised mainly of bones, around two hundred and six of them in an adult to be specific. However, there is a good amount of cartilage, joints, and ligaments, which all together make up around twenty percent of a person's body mass. As the environment is involved in bones' health, the five major functions of bones are to support the human body, provide a balance of locomotion, provide protection, create blood cells, and store calcium and phosphorus [17]. In fact, phosphorus is an individual element that can be combined in a specific way to form phosphate, which our skeleton stores over ninety nine percent of it. Additionally, our bones store over ninety nine percent of our total body calcium [18].

The bone is a composite material, organic and inorganic, that represents as a storehouse for some minerals and elements. The inorganic components (minerals) of bones account for 65 percent of the total bone mass. The marrow located in some bones produce blood cells (red \& white) and minerals such as calcium and phosphorous. These blood cells and minerals are stored in and supplied from bones [19]. Calcium and phosphate are

contained in small crystals called hydroxyapatite $\left(\mathrm{Ca}_{10}\left(\mathrm{PO}_{4}\right)_{6}(\mathrm{OH})_{2}\right)$ [19]. The organic component of bones on the other hand, is primarily collagen providing bones their high 
tensile strength. Collagen consists of the three cell types found in bones and osteoid: osteoblasts, osteocytes, and osteoclasts [19]. Bone development and remodeling takes place on a continual basis, from the prenatal period and early childhood through adulthood, as well as during the healing process that occurs following bone fraction. As a living tissue, bone is persistently rebuilt in reaction to the body's requirement for calcium and for fixing harmed tissue [17].

The skeleton changes across the human life span. This is characterized predominantly by bone formation and growth throughout the childhood, and then followed by a gradual loss of bone density that begins in early adulthood that can accelerate significantly in older adults. The density of bone is modulated by a group of cells including osteoclasts and osteoblasts. Osteoclasts are multi-nucleated cells that resorb bone, whereas, osteoblasts are cells that refill the resorption cavities created by osteoclasts. This cycle of bone resorption followed by bone formation is referred to as remodeling [19].

Osteoclasts incur themselves to the surface of bone, therefore this creates a microenvironment underneath the cell which is referred to as the sealed zone. Within this zone, osteoclasts create an acidic environment that dissolves the bone mineral contents. Once the dissolving has completed, osteoclasts release enzymes to remove the remaining collagen matrix to complete the process of resorption [19].

Osteoblasts move to the resorption space and start to produce and to deposit organic matrix called osteoid, which is made predominantly of collagen, forming a scaffold in which minerals including calcium and phosphate begin to crystalize. Some active osteoblasts become trapped within the matrix that they secrete, and thereby become 
osteocytes. Some osteoblasts will undergo apoptosis, or will revert back to lining cells, which covers the surface of bone [19].

There is also a process where bone formation by osteoblasts occurs without prior bone resorption by osteoclasts. This results in an increase in bone mass and is referred to as bone modeling. Bone modeling promotes the growth of bones for maintaining bone strength [19].

There is no single bone from which whole living beings are manufactured. Rather, a wide range of sorts of bones, each with various capacities, cooperate to give the skeletal framework strength and effectiveness [17, 19]. Bones can be classified to five types according to the overall shape of the bone so each type can fulfill certain functions. The most common type of bone found in the body are the long bones. They can vary in length, but the key characteristic is that they are longer than they are wide. They also have a shaft made primarily of compact bone, called cortex, and two bulky ends which often contain spongy bone, called cancellous [19]. An adult skeleton consists mostly of bones from the upper and the lower extremities such as a humerus, radius, and the femur. Short bones have approximately the same length and width, which gives them a cube-like appearance. They consist mostly of cancellous that is covered by a thin layer of cortex. There are twentyeight short bones in the adult skeleton consisting of the tarsals and most of the carpal bones of the wrist. There are sesamoid bones, and these are embedded in tendons. There are four sesamoid bones in the adult skeleton, two patella (kneecap) bones, and two pisiform (carpal) bones. Flat bones provide protection to vital organs. They are mostly flat and curved, having a thin plate-like formation. They also contain a layer of spongy bone covered by a thin layer of cortex. There are thirty-six flat bones in the adult skeleton 
including some of the skull bones as well as the sternum and the ribs. Finally, irregular bones come in various funky shapes and sizes. These bones also consist of a layer of cancellous, covered by a thin layer of cortex. There are forty-eight irregular bones in the adult skeleton including the hip bones, the vertebra, the hyoid, and some of the skull bones [17].

Some anatomists categorize the skeletal system to two divisions of skeleton: the axial skeleton and the appendicular skeleton. Bones of the skull, vertebral column, and the thoracic cave belong to the axial skeleton; however, upper and lower extremities along with their girdles belong to the appendicular skeleton [18].

Geometrically speaking, a bone's structure is comparable to fortified cement; a composite material, where the calcium mineral is the solid and collagen filaments act like the rebar. This sort of fortification is intended to oppose pressure, as well as strain, torsion, and bending loads; supplying more overall strength coupled with ductility. This implies that the bone is not a static organ [19]. Bones are continuously geometrically and structurally changing as well as changing in mass, so they perform properly.

Most bones in the body exist, morphologically, in two forms: compact/dense (cortical) bone and spongy/trabecular (cancellous) bone. Cortical bones, approximately account for 80 percent of the human skeleton mass, are exterior protective portions of all bones. It is found in the shafts of long bones like a femur. The remaining approximately 20 percent of the human skeleton mass is the cancellous bone that is found inside ends of the long bones and have numerous pores [19]. The cancellous mass is dominatingly seen in the vertebrae, most of the flat bones, and ends of the long bones. Despite the fact that there is a less mass of trabecular tissue, it is an imperative segment in load transmission 
and energy absorption for the long bones and spinal segment. Porosity ranges in cortical bones between 5 percent and 10 percent, whereas, it ranges in trabecular bones between 75 percent and 90 percent [19]. The visual distinctions between a healthy bone and an osteoporotic bone are depicted in figures $3 \& 4$. Femur anatomy is illustrated in the following figure.
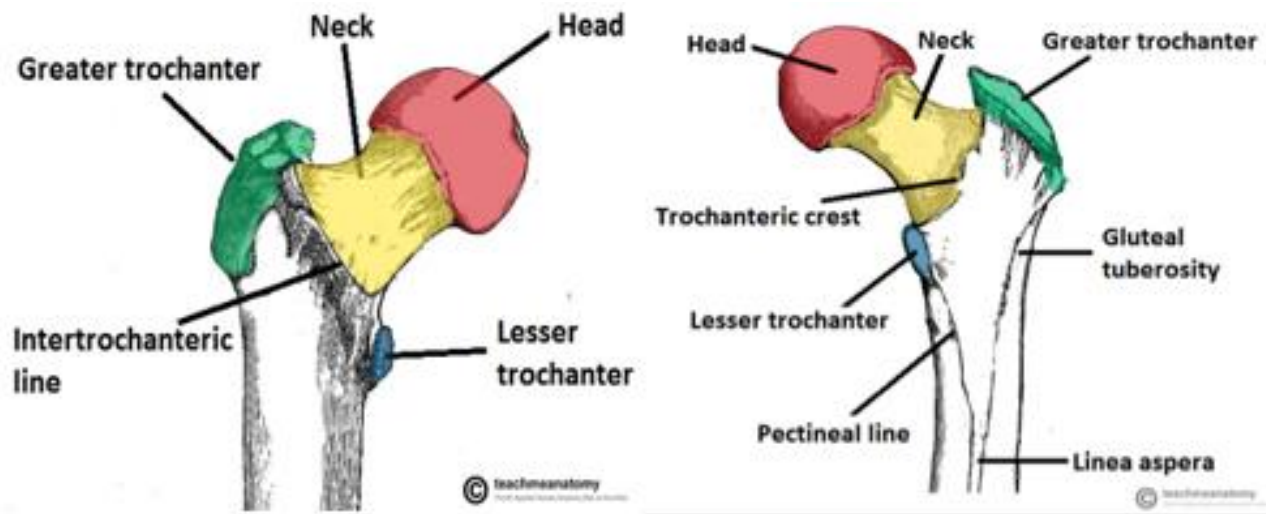

Figure 1: Anterior (left) and posterior (right) proximal femur anatomy [20]

Main components of the proximal femur are cortex and cancellous which involves trabecular bone and bone marrow as illustrated in the following figure. 


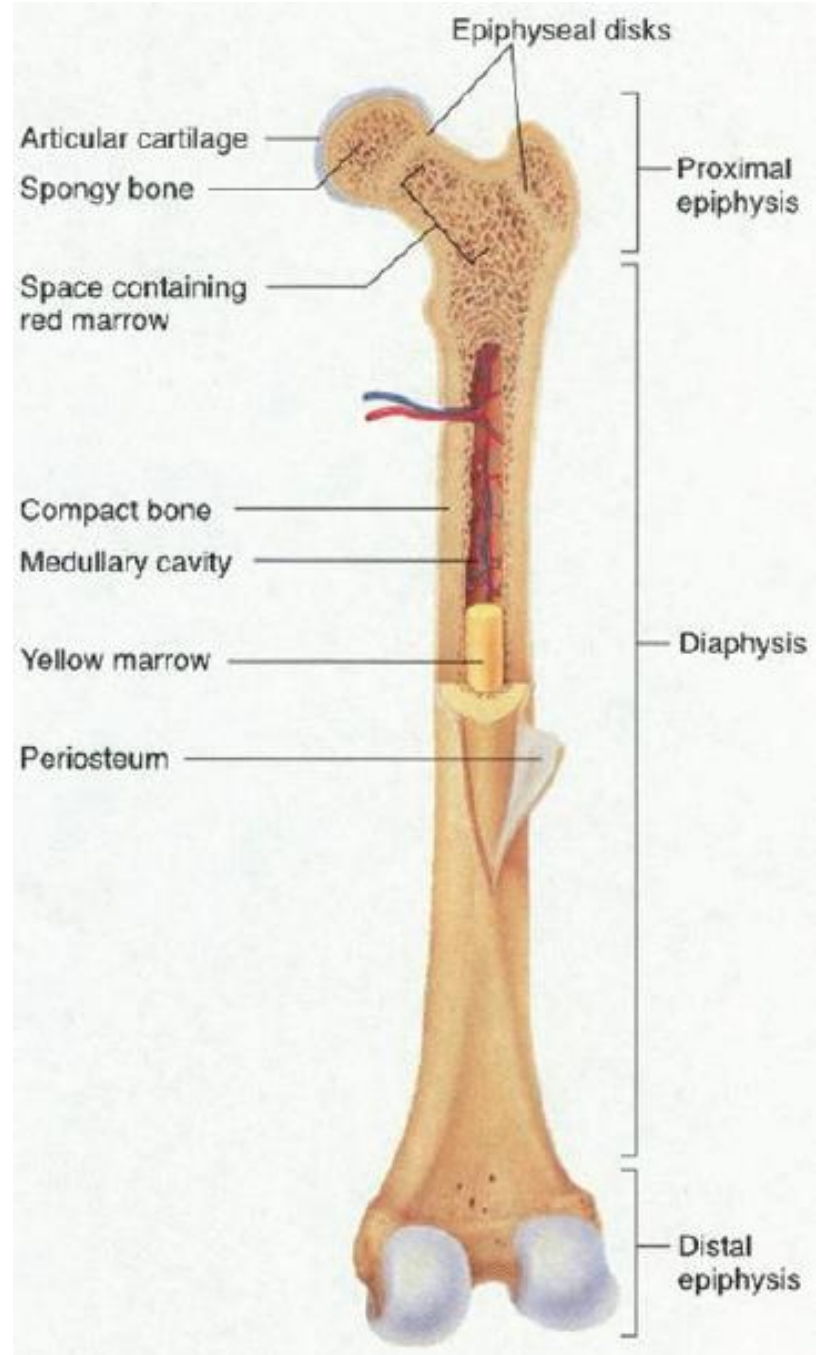

Femur

Figure 2: Schematic diagram of the femur structure [21]
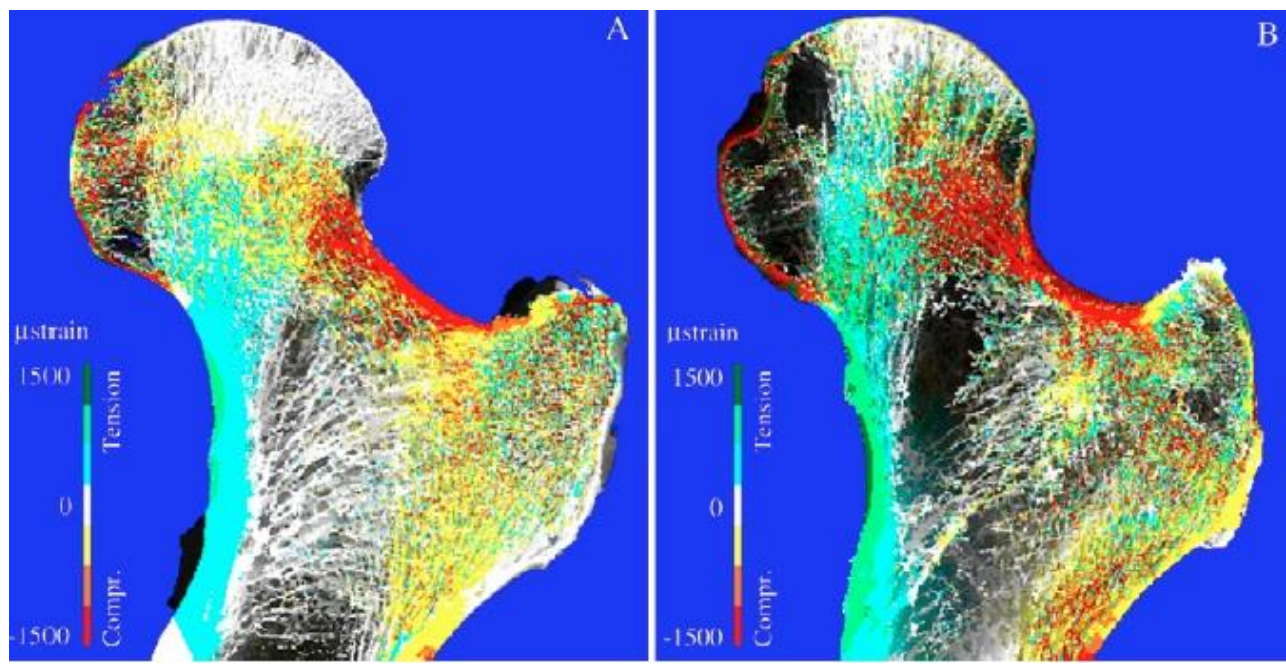

Figure 3: Healthy proximal femoral bone (left) vs. Osteoporotic one (right) [22] 

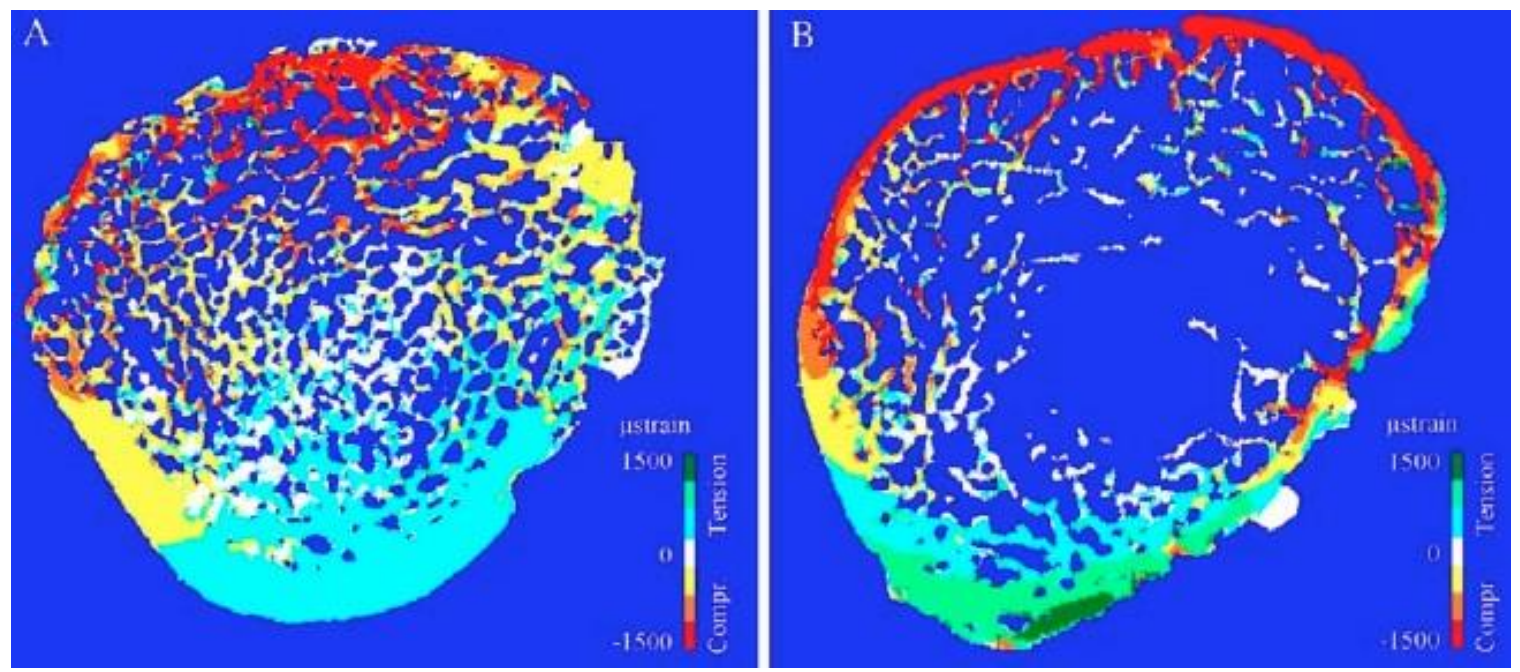

Figure 4: Maximal principal strain in the selected plane though the femoral neck in the healthy (A) and osteoporotic (B) femur [22]

Cortical and trabecular bone tissue are similar in properties at the bone-tissue (microscopic) level. Contrarily, their mechanical properties vary significantly at the continuum/apparent (macroscopic) level. The main assessment for the current research is performed at the apparent level behavior of cortical and trabecular bone. Structural/mechanical behavior of cortical and trabecular bone is typically represented by load versus displacement curve or by stress versus strain curve as illustrated in the following figure.

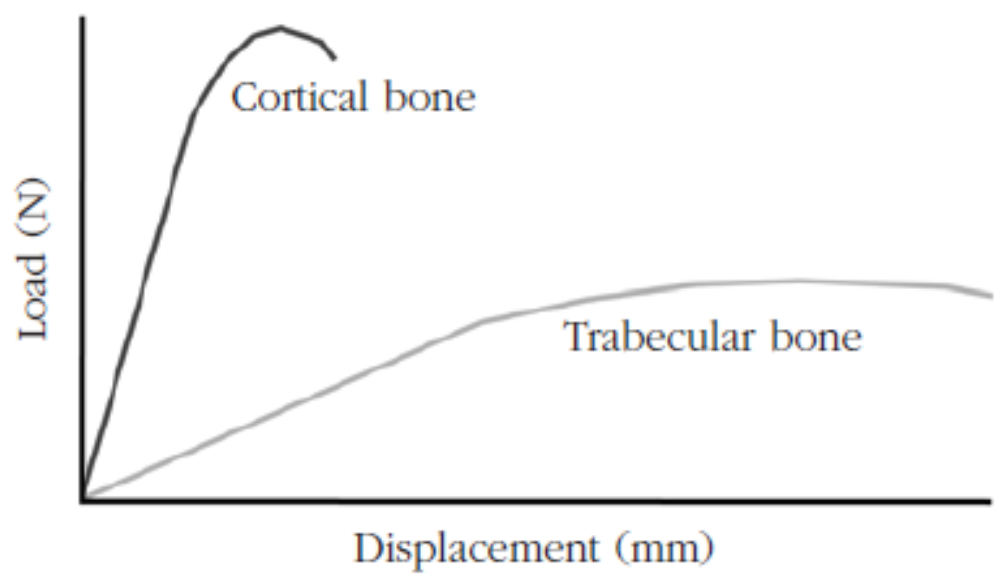

Figure 5: Typical apparent level load-displacement curve of biomechanical behavior characteristics of both bone tissue types: cortical and trabecular bone [23]

Regarding biomechanical behavior, difference between cortical and trabecular 
bone is obvious due to significant differences in morphological properties. The figure shows that cortical bone curve has a larger slope, which implies that it possesses a higher modulus of elasticity. This characteristic makes the cortical bone capable of incurring a larger stress with a low strain index, and hence it is stiffer than trabecular bone. In contrast, trabecular bone curve has a smaller slope, which implies that it possesses a lower modulus of elasticity. This feature also means the endured stress by the trabecular bone is lower, but with a higher strain index, and thus grants it a higher flexibility [23].

The stiffness represented by the slope of the elastic curve, is resulted from the division of variation in load by the corresponding displacement. Stiffness significantly varies with bone size and shape; however, when the size and shape are not considered, the load and displacement are converted to stress and strain respectively. The slope of the stress-strain curve in the elastic region is called Young's modulus, which is an intrinsic property of the material. The strength of the material is denoted by the ultimate stress value.

At the apparent level, the trabecular bone is anisotropic because of its trabecular structural organization, but not so much for any anisotropy of the bone tissue material. Failure stresses of trabecular bone significantly vary with respect to anatomic site, age, and loading direction. The most important parameters associated with that variation are volume fraction, architecture, and the tissue material properties [17]. Therefore, trabecular strength is heterogeneous, anisotropic, and asymmetric [17].

\subsection{Types of Bones:}

The types of bones or bone models that can be used in mechanical testing experiments are human cadaveric bones, synthetic bone surrogates, or animal bones. Generally, human cadaver bones are considered best to experimentally reproduce behavior 
that is being studied, particularly if that behavior is of a short duration and biological response is not important. The primary objective for using human cadaveric bones rather than animal or synthetic bones is to obtain optimal and more consistent representation for the real active human bones and specially to validate human FE models as well. Synthetic bones can be used in either destructive testing or non-destructive testing. However, destructive testing in which the failure occurs within the bone substance can be problematic because synthetic bone surrogates do not fail in the same way as real bone tissue. Nondestructive testing is carried out primarily for standardizing testing in order to determine some of the overall bone structural properties like stiffness, or to test an implanted medical device in an anatomically and geometrically realistic configuration. Fracture tests conducted upon animal bones are usually done after some type of in vivo experiment during which the bones are allowed to respond to sometime dependent factor such as aging, or drug treatment in order to determine the resulting change in bone's mechanical properties. Therefore, animal bones are often not appropriate because of geometric and anatomical differences between them and human bones [17].

\subsection{Biomechanics/Orthopedic Biomechanics:}

Biomechanics is the study of the structure and function of biological systems such as humans, animals, plants, organs, fungi, and cells by means of the methods of mechanics [24]. Biomechanics is closely related to engineering, because it often uses traditional engineering sciences to analyze biological systems. Some simple applications of Newtonian mechanics and/or materials sciences can supply correct approximations to the mechanics of many biological systems. Applied mechanics, most notably mechanical 
engineering disciplines such as continuum mechanics, mechanism analysis, structural analysis, kinematics and dynamics play prominent roles in the study of biomechanics [24].

Usually biological systems are much more complex than man-built systems [25]. Numerical methods are hence applied in almost every biomechanical study [26]. Biomechanical studies are carried out in an iterative process of hypothesis and verification, including several steps of modeling, computer simulation and experimental measurements.

The study of biomechanics ranges from the inner workings of a cell to the movement and development of limbs, to the mechanical properties of soft tissue and bones [27]. Biomechanics researches have wide variety applications from individual cells to whole organisms such as the investigation of the forces acting upon limbs, the aerodynamics of bird and insect flight, the hydrodynamics of swimming in fish, and locomotion in general across all forms of life. The biomechanics of human beings is a core part of kinesiology as well [27]. As a greater understanding of the physiological behavior of living tissues is expanding, researchers can advance the field of tissue engineering as well as to develop treatments for a wide array of pathologies [24, 28, 29].

Biomechanics is also applied to studying human musculoskeletal systems. Such research utilizes force platforms to study human ground reaction forces and infrared videography to capture the trajectories of markers attached to the human body to study human 3D motion [24]. Electromyography (EMG) system is used to study the muscle activation as well as to investigate the muscle responses to the external forces and perturbations [24].

Biomechanics is widely used in orthopedic industry to design orthopedic implants for human joints, dental parts, external fixations and other medical purposes. Bio-tribology 
is a very important part of it. It is a study of the performance and function of biomaterials used for orthopedic implants. It plays a vital role to improve the design and produce successful biomaterials for medical and clinical purposes such as tissue-engineered cartilage [24].

\section{$2.4 \quad$ Bone Health:}

Typically, the bone mass reaches its maximum level by the early ages of 20. Aging advancement is one of the most natural factors influencing the bone mineral metabolism process, which is described by low bone mineral density (BMD) and low bone mineral content (BMC)(Figure 6). The bone metabolism process becomes unbalanced in the elderly due to advanced aging in normal conditions. Thus, the bones become fragile due to decreased level of osteoblast and the increased level of osteoclast as well. The bone becomes vulnerable and susceptible to breakage from being exposed to any kind of abnormal loading, overloading, or unusual movements [5].

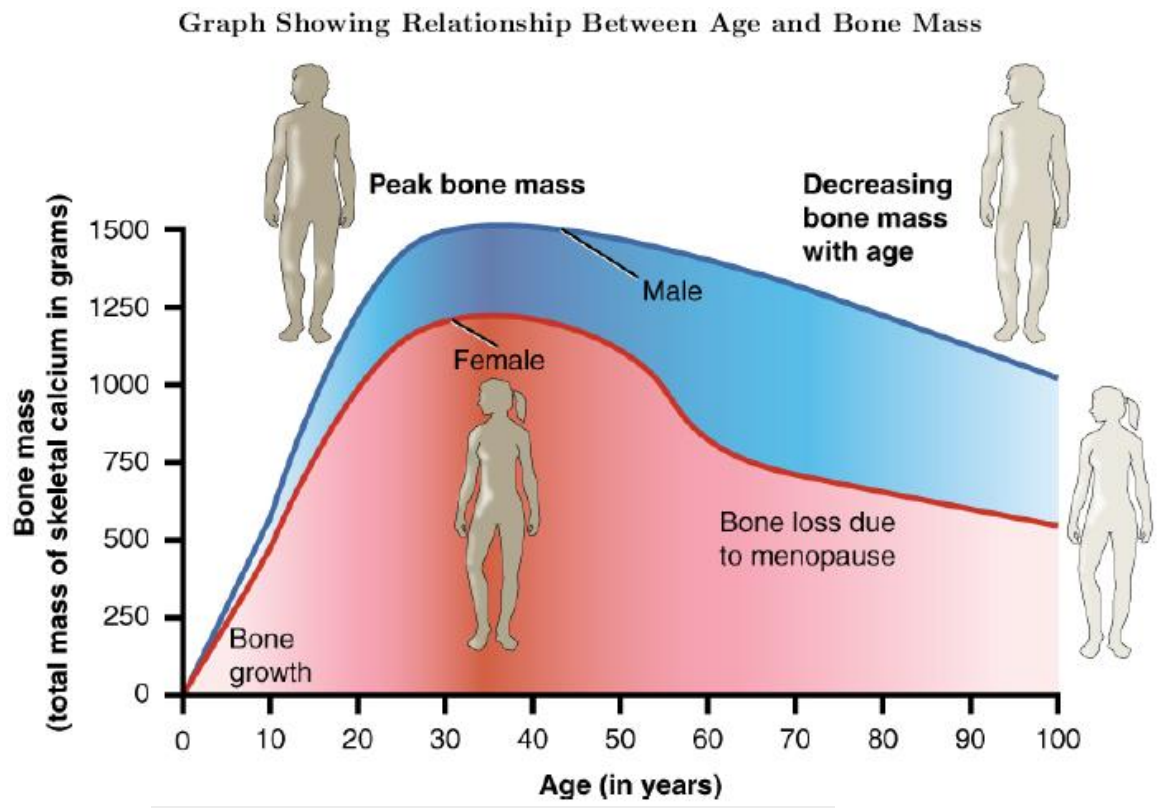

Figure 6: Women lose bone mass more rapidly than men [30] 
Osteoporosis is defined as a skeletal disorder denoted by low bone density, resulting in weakened bone strength, which overtime increases susceptibility to fracture. Bone density and bone architectural quality are the two main determinants of bone strength. Bone density is defined by the number of grams per unit of area or volume and it's compared with peak bone mass and amount of bone loss (average of young, healthy adults) as measured by dual-energy X-ray absorptiometry (DXA). Architecture, turnover, damage accumulation, and mineralization are references of bone quality. Normally, the persistent loss of bone is aggravated in women beyond menopause and in men with andropause $[17,18]$. Primary osteoporosis is described by insufficient metabolic bone process, which expresses low bone mass, and by a deteriorated architecture of bone tissue developing a fragile bone and hence increasing the fracture risk. Secondary osteoporosis takes place by bone loss that is significantly caused by contributory chronic condition or conditions. Medications (steroids) and nutritional (alcoholism) deficiencies are examples of factors leading to secondary osteoporosis. The World health organization (WHO) has set the level of osteoporosis for bone density that is 2.5 standard deviations (SDs) or more below the young adult mean value $(\mathrm{T}$-score $<-2.5)$. The patients with bone density SDs range (T-score -1 to -2.5 ) are classified to have osteopenia [31]. In normal conditions, the bone mass of people aged at 50 or older begins to decrease because of the deficiency in bones' metabolism process, which is denoted by indices of bone turnover processes. Thus, in the beginning process of bone loss, the condition is called osteopenia. Gradually with deficiency of any bone components, the bone acquires more deterioration and thus the condition is called osteoporosis, which involves substantial fragile bones and thus bones 
become susceptible to fracture. John A Kanis plotted the categorization of T-score levels in the following figures [9].

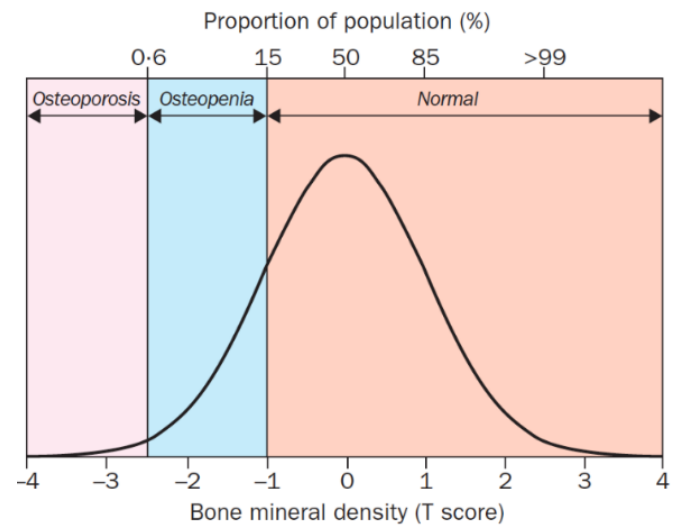

Figure 7: Distribution of bone mineral density in healthy women aged 30-40 years [9]

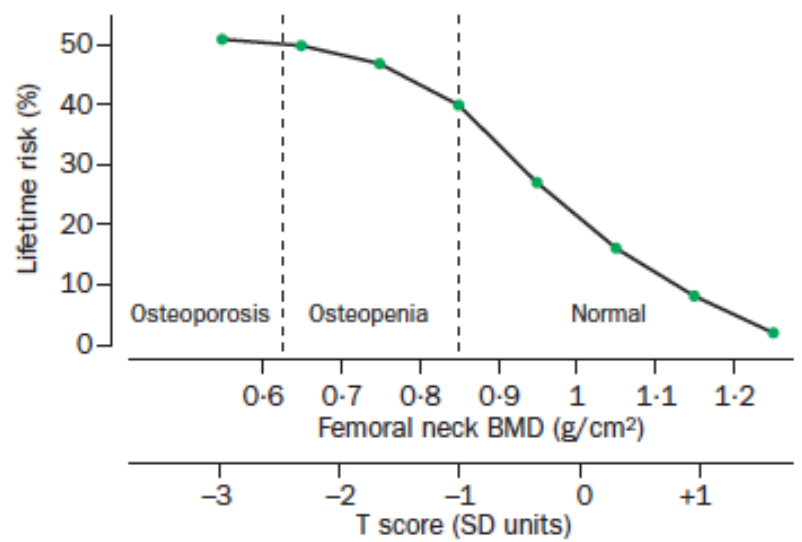

Figure 8: Remaining lifetime risk of hip fracture in women aged 50 years, according to bone mineral density (BMD) or T score at the hip [9] 


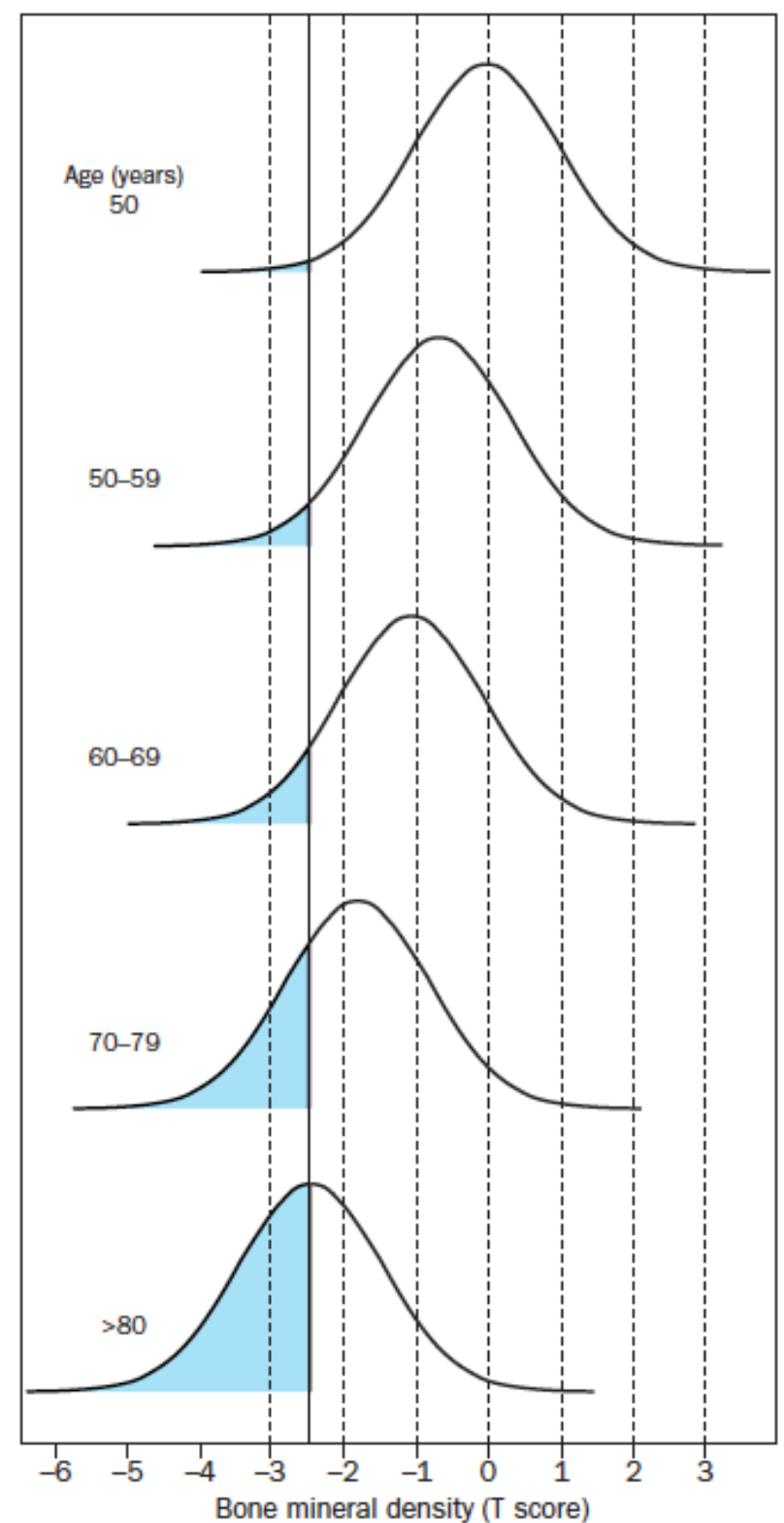

Figure 9: Distribution of bone mineral density in women of different ages, and the prevalence of osteoporosis (blue) [9]

Augat et al (1998) have documented those measurements from nondestructive tests performed over bone cubes from the spine, the calcaneus, the distal, and the proximal femur in multi-directions. The directions are called Cephalo-caudal (CC) (vertical), Anteriorposterior (AP) (front to back), and Medial-lateral (ML) (side to side). The maximum obtained elastic modulus value was for the proximal femur in $\mathrm{CC}$ direction and thus it's less resistant to fracture in $\mathrm{ML}$ and $\mathrm{AP}$ directions than in $\mathrm{CC}$ direction at particular 
anatomical points. Also, the proximal femur has the greatest values of the (BMD) comparing to other bones at different anatomical locations. Moreover, they have obtained that (BMD) does correlate significantly with mechanical properties of trabecular bone where the mechanical anisotropy of trabecular bone is minimal. They also correlate greatly in the principal loading directions under the condition of dependence on the anatomical locations as well, otherwise they correlate insignificantly [32]. Some researchers have concluded that external loads, applied during normal daily life, have a significant relationship with morphology parameters [33]. Results have successfully demonstrated how the normal daily loading condition is distributed in an osteoporotic and a healthy vertebra by combined introduction of parallel supercomputers, micro--CT, and micro-FEA. Results have confirmed that osteoporotic vertebral structure is well adapted to normal daily life loading, but not adapted to collateral "error" loads. Hopkinson et al (1998) classified a group of elder patients who had hip fractures according to the type of fall, type of fracture, and the activity at time of fall. As shown in that study, the most frequent type of falling incidence denotes to the side falling versus intra-capsular fractures followed by side falling versus extra-capsular fractures. The study also has shown that the most repetitive falling incidence has no rotation of femur [34]. Therefore, the point of interest for this project will be on falling to the side, as the proximal femur is more susceptible to fracture in this direction.

The hip joint is composed of the proximal femoral head that articulates with the pelvis at the acetabulum. It is one of the most important joints in the human body for mobility. The load distribution from normal daily activity transferred into the hip through the femur neck, where the majority of the force is concentrated. In order to ensure support 
to the axial skeleton, the femoral neck is composed of arching trabecular bone. Trabecular bone provides increased support to the outer cortical bone similar to the action of flying buttresses in architecture. The cortical bone is relatively thin in the area of the proximal femoral neck and gradually thickens distally.

Hayes et al. [1995] determined the stress distribution in a normal and osteoporotic femur caused by tow loading configurations: (1) gait, and (2) a fall to the side with impact onto the greater trochanter. They later investigated the structural significance of cortical and trabecular bone in the proximal femur. The cortical and trabecular bone supported approximately constant percentage of total load in all load cases but differed based on location. Cortical bone supported $30 \%$ of the load at the sub-capital region, $50 \%$ at the midneck, $96 \%$ at the base of the neck and $80 \%$ at the intertrochanteric region [35].

Bouxsein et al. [1996] experimentally investigated the effect of impact direction on the failure load of the elderly proximal femur. They used thirty-three cadaveric femurs as assigned randomly to three groups of 11 and tested at one of the three loading angles, $0^{\circ}$, $15^{\circ}$, or $30^{\circ}$ representing a fall on the hip rolled slightly forward, to the side, or rolled slightly backward, respectively. Results revealed that failure load decreased by $24 \%$ as the loading angle changed from $0^{\circ}$ to $30^{\circ}$, and therefore the impact direction is critically related to the hip fracture [36].

Felix Eckstein et al. [2006] mechanically tested 140 human cadaveric proximal femurs in a side impact configuration to investigate the geometric factors and failure loads level associated with the distribution of cervical (femoral neck) versus trochanteric hip fractures. Those femurs belonged to 77 females: mean age, 81.7 years; 63 males: mean age, 79.1 years. Results revealed that cervical fractures were higher in females $(74 \%)$ than in 
males $(49 \%)$. Trochanteric fractures were more pronounced at high failure loads, whereas the femoral neck fractures prevailed at the lowest structural mechanical strength levels. The best predictor of fracture type among geometric variables was the neck-shaft angle [37].

\subsection{Diagnostic Techniques of Osteoporosis:}

Diagnosis and treatments for osteoporosis are mostly carried out by using multi medical imaging scanning systems. Early studies described the DXA bone scanner as the golden technique is used to determine and detect whether people have osteoporosis by measuring the BMD. It is a simple $\mathrm{x}$-ray scanner with an $\mathrm{x}$-ray tube, which measures the bone density quantitatively. This implies that DXA could not measure the bone microstructure or quality that is believed to influence fracture risk. Consequently, neither normal BMD guarantees that the fracture will not occur just that the risk is reduced, nor does BMD measurement at the osteoporotic range guarantee that the fracture will occur but it's more likely [38].

Subsequent studies developed a technique called Quantitative ultrasound (QUS) of Heel and Tibia [39]. They have evaluated the correlations among 1) strength of the proximal femur; 2) BMD of femur, heel, and tibia; and 3) QUS of the tibia and heel. They concluded that there was a great correlation between strength of the proximal femur and femoral BMD and heel BMD $\left(\mathrm{R}^{2}=0.78-0.92\right)$. Another strong correlation observed from Broadband ultrasound attenuation (BUA) and speed of sound (SOS) of the heel to femoral strength (0.7 and 0.67 respectively) [39].

Recently, within each anatomical site, regression analysis was carried out for "critical" subgroups of specimens. Choosing of "critical" specimens for each site was 
performed based on the criteria that their trabecular volume relative to the total volume (BV/TV) was between the $25 \%$ quartile and the $75 \%$ quartile of a site's $\mathrm{BV} / \mathrm{TV}$ distribution. When BMD was used as the only determinant for the mechanical properties of the bone, it could not identify a subgroup of "critical" specimens as a normal or osteoporotic [40].

In 1895, Wilhelm Conrad Roentgen invented X-rays [41]. He was working with the tube that consisted of a glass envelope (bulb) with positive and negative electrodes encased in it. A high voltage was applied after he evacuated the tube from air, and as a result a fluorescent glow was emitted. Although the tube was very well covered, the rays passed through various objects placed in their path, so he captured them. The historic turning point of this discovery in regard to medicine is how the body could be watched with a nondestructive way [41]. In 1961, William Oldendorf built a prototype of an automated scanning technique [42]. That prototype consists of an X-ray source and a mechanically coupled detector rotated around the object to be imaged. By reconstructing the image, this instrument could get an X-ray picture of a nail surrounded by a circle of other nails [43]. In 1963, Allan MacLeod Cormack developed the first tomographic device that experimentally demonstrated the best image reconstruction without issues [43]. Thereafter, the convergence between X-ray technology and the emergence of computers enabled Godfrey Newbold Hounsfield in constructing the first Computed Tomography (CT) scanner [43]. Cormack, who developed the theoretical application of the CT scanner, shared the Nobel Prize for Physiology or Medicine in 1979 with Hounsfield, who built a whole CT scanner and developed its practical application [43]. The CT scanning concept is represented in two steps. The first step is about generating X-rays that are launched from 
a point source generator to pass through a specimen of interest in the form of rotational motion in equal steps of angles until completion of a whole cycle. Typically, every single pass of an X-ray beam through the specimen provides quantitative information of particular region like the tissue density, and that beam is detected by a large $2 \mathrm{D}$ planar which is placed across the generator. In the second step, all 2D raw images are exported from the CT scanner to the computer in order to be reconstructed, and then a whole 3D image of the volume is generated, which consists of a large stack of 2D reconstructed images [43].

Various types of CT scanners were made at different levels of resolution for evaluations of bodily tissues. Knowing the range of tissues architecture sizes (human bone vs rat bone) is required so the optimum type of a CT scanner could be determined. Additionally, the level of application (macroscopic vs microscopic) is a controlling factor over the machine selection. Some examples of CT-scanners include the peripheral quantitative computed tomography (pQCT), clinical scanners like multi-detector computed tomography (MDCT), and high-resolution computed tomography (HRCT) [44].

The pQCT scanners technically image the peripheral skeleton such as radius and tibia [45]. While the scanner detectors rotate axially around the region of interest radially, the body must be fixed. The negative point about this scanner is its limitations in regards of specimen size and the scanning resolution that ranges between 0.05 and $0.15 \mathrm{~mm}$ [46].

The MDCT is used in most diagnostic imaging departments to scan the upper torso such as spine and hip bones. It is designed with two dimensional arrays of multi-detector elements, which permit CT scanners to acquire multiple slices simultaneously and greatly increase the speed of CT image acquisition. MDCT provides a lower resolution (250micro- 
$\mathrm{m}$-300micro-m) than pQCT, therefor it does not image spatial resolutions of trabecular dimensions [44].

Mechanical properties of trabecular bone have been precisely provided by new developments in 3D structural analysis through designing the HRCT scanners [40]. This scanner has been the best tool to produce 3D images at the highest resolution level ( 0.3 micro-m-100 micro-m).

J. Kabel et al [1999] have diagnosed the mechanical quality of cancellous bone through addressing the significance of trabecular bone connectivity. Elastic properties of trabecular bone comprised volume fraction $\left(\mathrm{V}_{\mathrm{V}}\right)$, connectivity density $\left(\mathrm{b}_{1 \mathrm{~V}}\right)$, and surface density $\left(\mathrm{S}_{\mathrm{V}}\right)$. The volume fraction variable was determined as the optimum morphological variable since it had the highest correlation level [47]. W. Pistoia et al. [2002] have estimated the failure load of a distal radius using micro-FE (micro-FE) analyses with models based on three-dimensional peripheral quantitative computer tomography (3DpQCT) images. The results confirmed that there was a better prediction of the failure load with the application of micro-FE, which delivered 0.75 of correlation with the experimental failure load, than the prediction with the application of DXA which delivered 0.57 of correlation with the experimental results [48]. The HRCT technology was operated by Later, D. Ulrich et al [1999] who have measured 3D structural indices and elastic constants of human cancellous bone at different anatomical sites and next have obtained the predictive value of those indices for elastic properties of bone. The structural indices, which calculated from the segmented 3D images, are (BV/TV), relative bone surface (BS/TV), trabecular thickness (Tb.Th), trabecular separation (Tb.Sp.), trabecular number (Tb.N), and mean intercept length (MIL) ratio. On the other hand, those images were used for micro 
structural analysis to calculate Young's moduli, shear moduli, and Poisson's ratios. The maximum correlation $\left(\mathrm{R}^{2}\right)$, at femoral head, substantially jumped from $53 \%$, for the structural index BV/TV alone, to 92\% when Tb.Sp., and MIL were also included [40].

A customized HRCT scanner called ACTIS scanning system was used in the current research as shown in the following figure.

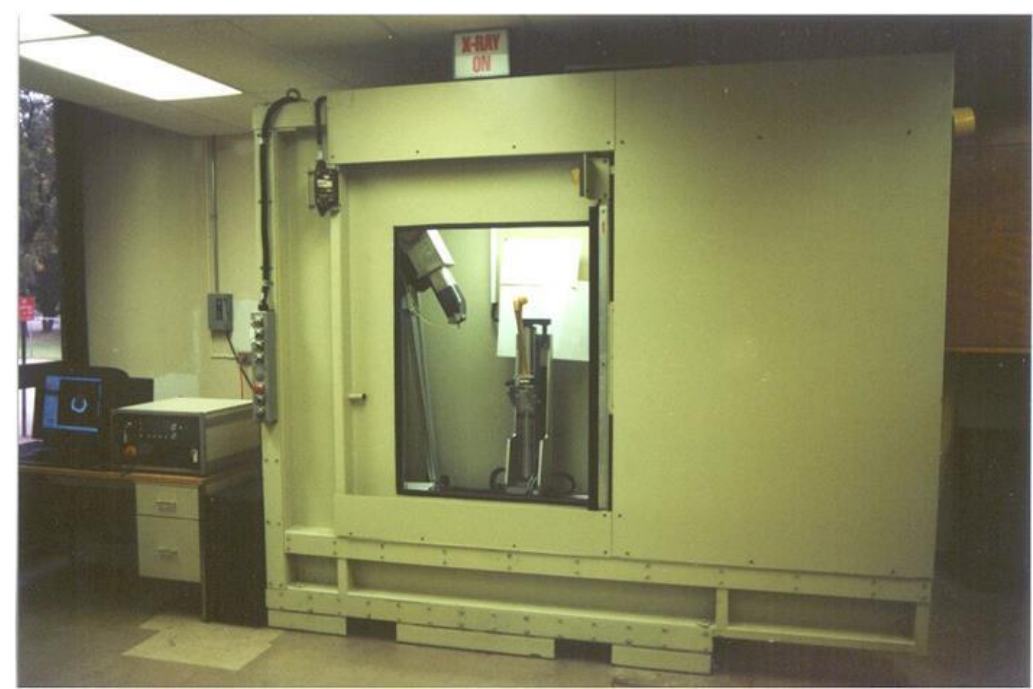

Figure 10: ACTIS high resolution computed tomography (HRCT) laboratory scanner used in the current study

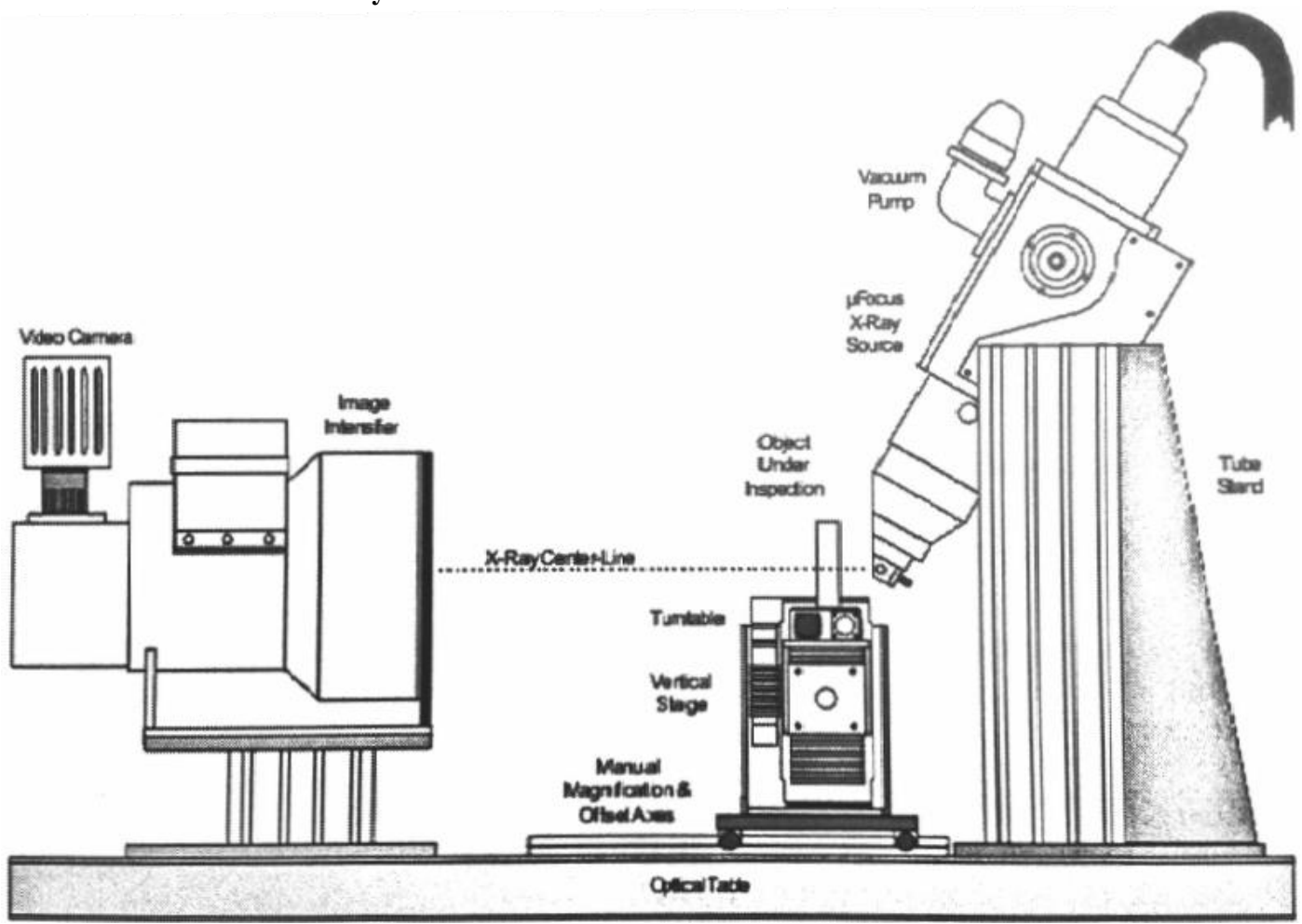

Figure 11: System components of key ACTIS scanner [50] 
This system is a variable power CT system (ACTIS 150/225 system, BIR Inc., IL). The source and image intensifier used in this research system are a micro-focus X-ray and an X-ray detector.

Some calibrations, aligning, adjustments, and then measurements procedures are performed prior to scanning process. Typically, in order to obtain the best spatial resolution, specimens are positioned where they fill the widest range of the field of view including some factor of safety margin such as $90 \%$ of field of view. The maximum magnification possible is determined by calculating the source to object distance (SOD), source to image-intensifier distance (SID), and the usable face of the image intensifier width. However, SOD depends on which scanning mode is applied as there are two scanning modes, centered, and offset as shown in the following figures.

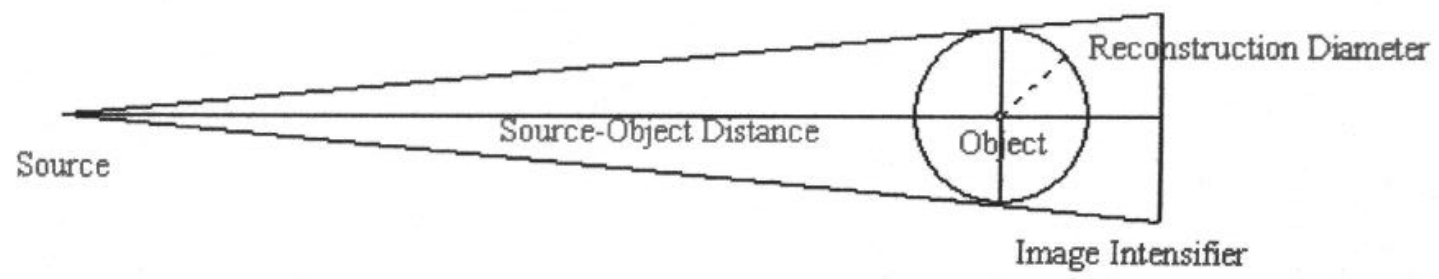

Figure 12: Centered mode scanning [50]

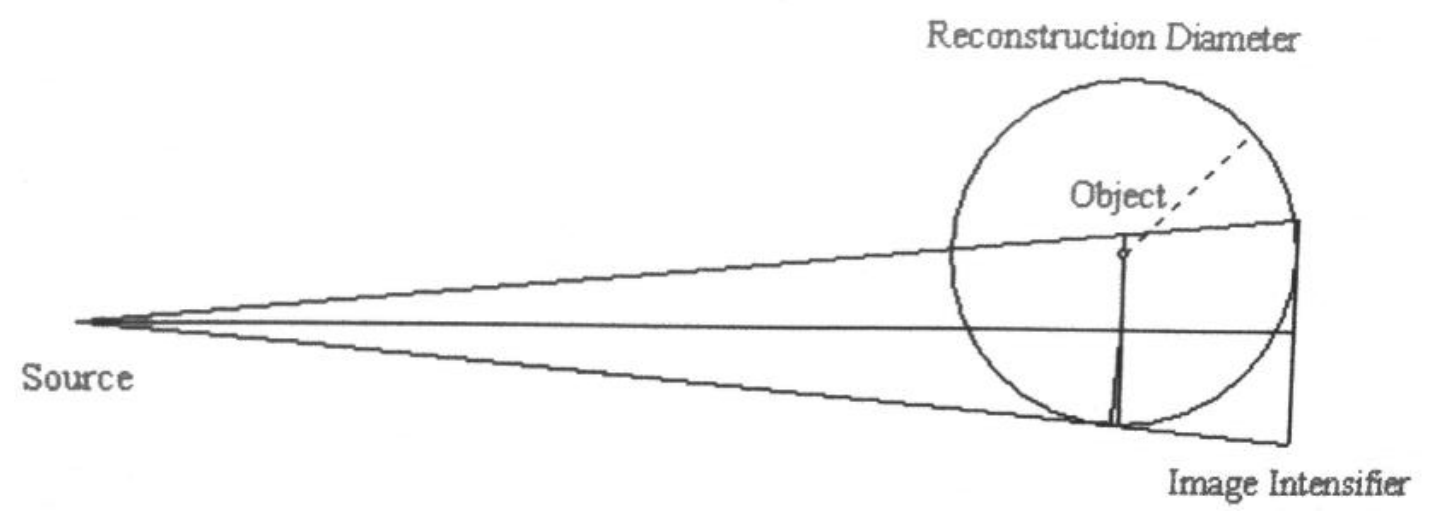

Figure 13: Offset mode scanning [50] 


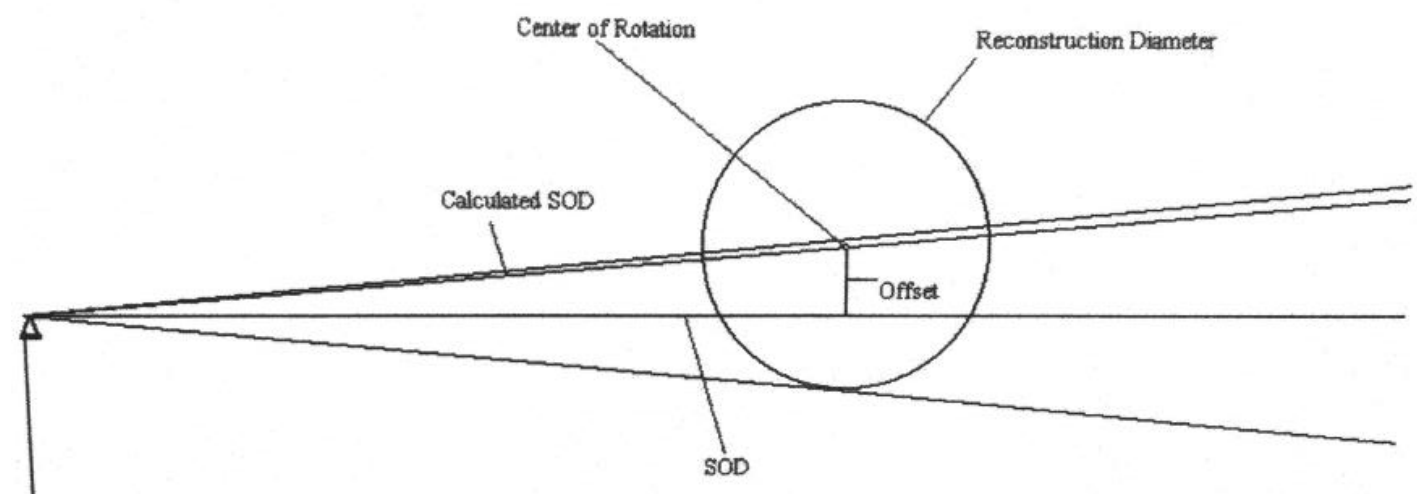

Figure 14: Sample Mount Gantry Setup [50]

\subsection{Image formats and segmentation:}

Image segmentation is a subfield of artificial intelligence and computer vision. It is typically used to locate objects and boundaries in images. It is mostly used for partitioning an image into its regions-based on some criteria where the regions are meaningful and disjoint, like finding a cat in an image and isolate it [49].

The image can be divided or partitioned into various parts called segments. Prior processing the image, it is a great idea to exclude the regions in the image which do not contain any information. By dividing the image into segments, the important segments can be used for processing the image. An image is a collection or set of different pixels. The pixels that have similar attributes are grouped together by using image segmentation. As a result, image segmentation creates a pixel-wise mask for each object in the image. In other words, multiple segments are recreated for identifying everything in the image [49]. There are many applications where image segmentation is very useful such as remote sensing for earth's surface, medical images for human or animal bodies, or roads captured by drones.

Image files formats, generated by these scanners, include but not limited to digital imaging and communications in medicine (DICOM) and tagged image file format (TIFF) [50]. The HRCT scanner of the current project generated a raw image, which is then 
reconstructed into a stack of $2 \mathrm{D}$ monochromatic and raster graphics TIFF images. TIFF images have specifications of resolution grid of cubic volumetric data, voxel grid. The generated stack of TIFF images can be converted to 3D solid model for analysis in a computational software. The HRCT scanning process is a very useful technique since it is based on a specific patient nondestructive study methodology. It also allows spatial visualization, density resolution, as well as precise measurements of certain parameters like volumetric spaces [51].

Images processing techniques are required in order to transfer images from the HRCT to FEA, one the best is called Materialise's Interactive Medical Image Control System (MIMICS). MIMICS was used in the current research to calculate surface 3D models from stacked image data such as CT, HRCT, MRI, Confocal Microscopy, X-ray and Ultrasound, through image segmentation [52]. Mimics program is a Materialise's software for processing medical images and creating 3D models as illustrated in figure-15 [53]. Mimics uses 2D cross-sectional medical images such as from CT and MRI to construct 3D models, which can then be directly linked to rapid prototyping, $\mathrm{CAD}$, surgical simulation and advanced engineering analysis. Materialise 3-Matic is a design and meshing software for anatomical data. The graphical user interface for 3-Matic is illustrated in figure-16. Mimics 3-Matic program includes segmentation, meshing, measurement tools to end-use FEA compliant with standard FEM solvers. The triangle surface properties were used for volume mesh algorithms to control the quality of the mesh form and density resulting in a well-developed tetrahedral volume model as illustrated [53]. 


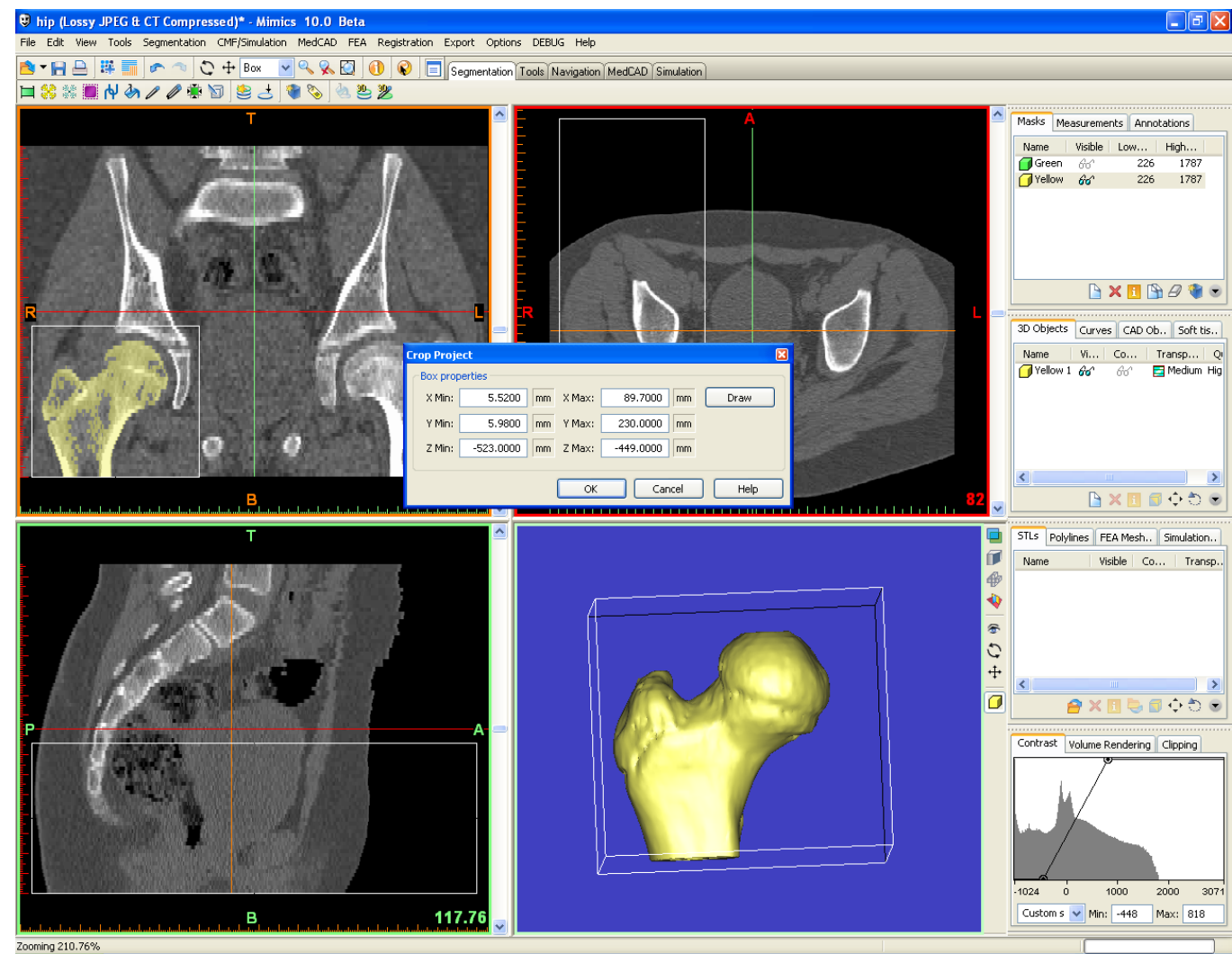

Figure 15: Stack of 2D images being converted to a 3D surface in MIMICS program [53]

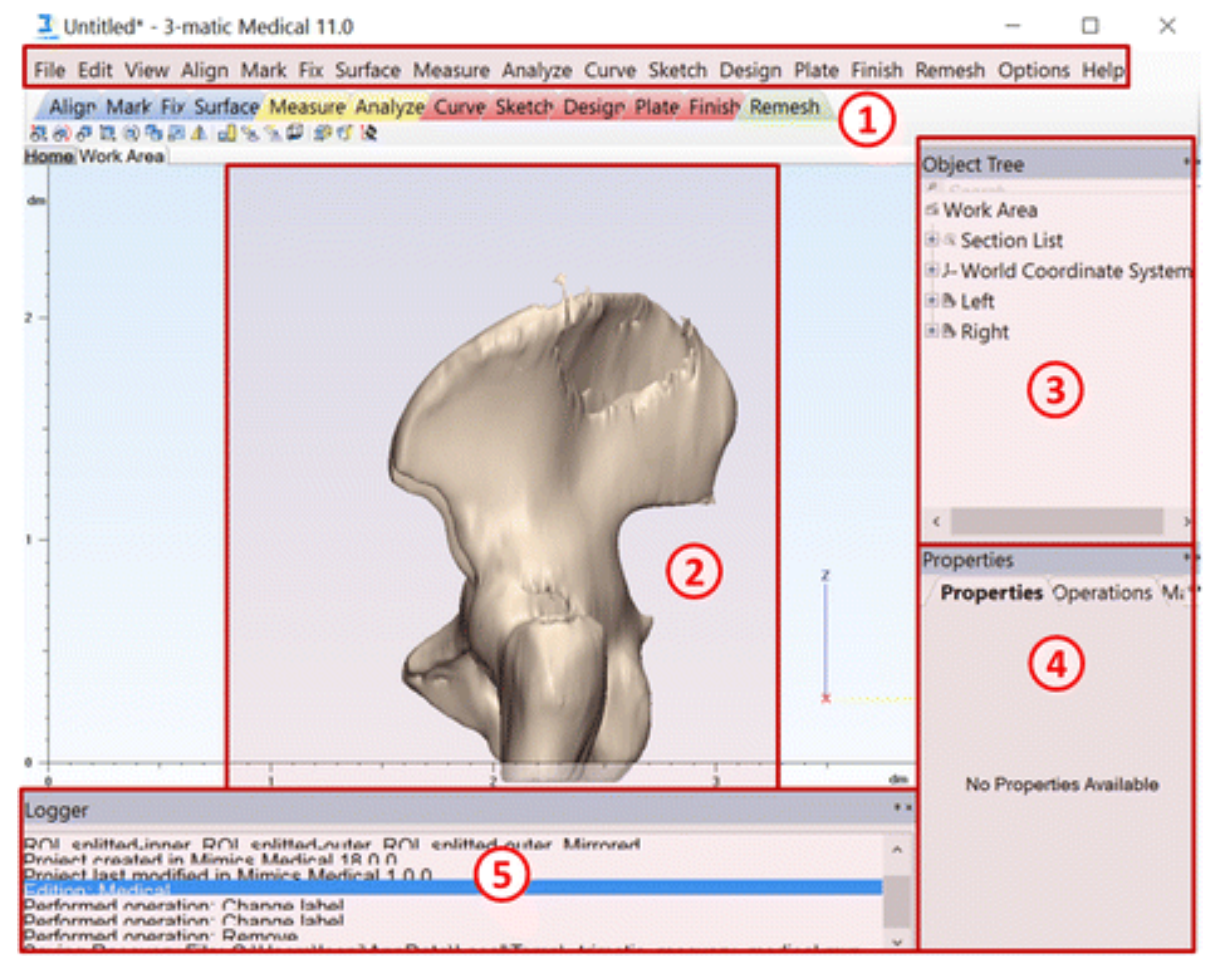

Figure 16: Main window overview of 3-matic program. Featured are the menu toolbar (1), 3D view (2), object tree (3), properties (4), and the logger (5) [54] 
The process starts with stacking of the entire 2D images, and segmentation thereafter. Image stacking is the sequential importations of the total number of individual voxel scan slice data. The voxel scan slice thickness is based on the scanner set resolution for capturing the test article details. Image segmentation describes the process of determining the continuously connect entities within and between the image voxel data based on a thresholding of gray scale values [53].

The thresholding segmentation technique is known as Dynamic Region Growing within the Mimics work environment. Within Dynamic Region Growing command, thresholding does not need to be done first. Instead, Mimics creates what is known as a Mask based of how surrounding pixels compare to a selected data-point's gray value. The Mask is a solid 3D volume of connected voxels maintaining continuity around and between adjoining voxels attached at the edges or on their square faces [53]. Once the images are properly segmented, a finite element model for mechanical simulation can be created. Since the model is based on medical anatomical image data, the resulting volume is a detailed specific representative of a real anatomical structure to be analyzed [53].

The elements that comprise the 3D structure must have certain geometric qualities and constraints that enable the compiler of the analysis software to correctly process a solution, in order to prepare the Mimics volume mesh model for simulation. Node and edge interface, of the adjoining mesh elements, is the most important thing in creating the FEM for bone in biomechanical modeling. The mesh needs to have properly connected, and clearly defined properties. If this process done improperly, the model will be unsolvable or inaccurately converge on a solution $[52,53]$. 
Once the qualified meshing requirements have been fulfilled, and the 3D volume mesh has been created, the model could be exported as an input file format to be used directly in commercially available finite element analysis software [52, 53].

\subsection{Brief History of Finite Element Analysis (FEA):}

In the 1940s, prior to the origins of true finite element analysis, Courant obtained approximate numerical solutions of stresses in variational form by using piecewise interpolation (or shape) functions over triangular sub-regions $[55,56]$. After Courant, Argyris published a significant landmark paper in the history of structural mechanics in the 1950s [55]. He developed the matrix theory of structures for discrete elements. This innovation goes on to show that this is only a particular case of the general continuum in which stresses and strains have been specified, and thus leads to the concept of flexibility and stiffness. In the meantime, the stiffness matrix in global co-ordinates was derived by Turner. This pioneering paper demonstrated that the triangle was not only simpler to handle than the rectangle, but later it was used as the basic building block for calculating stiffness matrices for plates of arbitrary shapes. Turner's invention has been given full credit as it has been outlined in Clough's paper since the latter invented the name finite element method [55]. Clough showed his extension of Turner's work from 1957 onwards to the calculation of stresses and the verification that for known geometries and loading these stresses converged to the corresponding analytic solution.

The FEA is a numerical procedure used to tackle engineering issues utilizing a variety of scientific methods. The name of FEA originates from the way that the technique subdivides a bigger problem into littler more straightforward parts that are called finite elements (FE). Analytical solutions are found for the mathematical formulas that represent 
these FEs. Then, those mathematical formulas are gathered over into the bigger arrangement (array) that contains the whole problem which was then named "Matrix Structural Analysis" (MSA). MSA, variational approximation theory, and the digital computer are three components formed the Finite Element Methods (FEM) [57].

To know how the proposed structure will behave under a load, the equations describing the distribution of structural stresses are known, but they can't be directly solved for a complicated shape such as a bridge. The finite element method takes the single complicated shape to replace it with an approximately equivalent network of simple elements such as triangular shapes or quadrilateral. The overall pattern of elements is referred to as the $\mathrm{FE}$ mesh, and this pattern will be unique to each new problem. The accuracy of the calculation depends on the number of elements chosen for the mesh. The more elements the model has, the smaller each one will be and the more accurate the results. This concept is called "convergence" as the numerical solution "converges" on an exact solution. On the other hand, more elements mean more calculations to be done. Therefore, adequate accuracy is obtainable by having enough elements that is considered an acceptable representative of the physical model [57].

FEA is especially important to examine those conditions which cannot be researched straightforwardly by experimentation and in this way, gives significant data that can't be acquired in more straightforward ways [58]. FEA can likewise assist us with understanding how a structure may act precisely under different conditions that can't be repeated tentatively [58]. Concisely, the technique utilizes a serial of elements that can be an assortment of shapes, and those are attached at nodal dots, to computer-produce an irregular morphological structure, for example, a complex human bone. The elements can 
be given properties that are representative of the tissue properties that little regions of the biological structure have, either depending on estimations that have been made of the properties of the material, or in those cases in which the properties have not been estimated, assessing them dependent on what we do know.

In the early of 1970s, the FEM was applied in the field of biomechanics as well as those applications have extended from the molecular level to cellular, tissue, and organ levels [55]. Dramatic increases of FE applications in biomechanics were denoted to greater accessibility of commercial software and to improved computing platforms to the research communities. Numerous FEA commercial software programs include but not limited to ANSYS and ABAQUS were developed and released in the period (1970s-1980s) as the $1^{\text {st }}$ and the $2^{\text {nd }}$ generation FEA Codes \& pre- and postprocessors respectively [55]. Computational modeling of discretizing arbitrarily complex geometries has become more prevalent in 1990s because of resiliencies afforded by the unstructured and adaptive meshing technique [57]. The efficiency of this technique came from its ability to perform local meshing enrichment and restructuring instead of global meshing regeneration [59]. Differentiation between FEA solvers is based on advances in some features such as capacity to handle large-scale models, and interface with other volume rendering software by accepting a variety of digital solid volume file formats [55]. An example of discretizing a circle to FE is illustrated in the following figure.

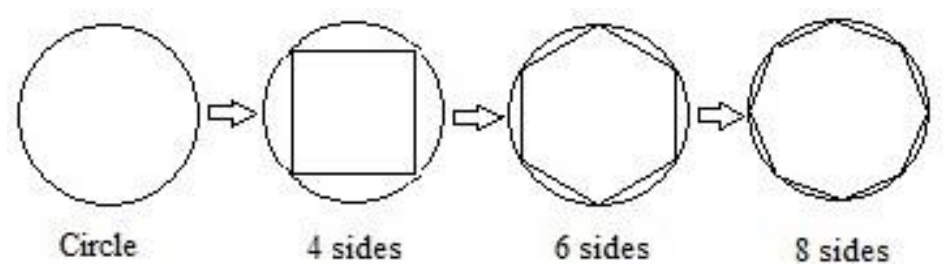

Figure 17: Discretization a circle from 4 sides to 8 sides polygon 
Finding the perimeter of a circle is possible by approximating its area by the area of a polygon. A discretized segment, which is called a "finite element", is represented by the polygon sides. As the number of sides on the polygon increases, the approximation gets closer to the value of the true perimeter. The overall pattern of elements becomes known as the finite element mesh for the given geometry.

\subsection{Introduction to FE Modeling Procedure:}

A general-purpose finite element analysis software package called ABAQUS, was used in the current research. Every complete FEA is composed of three stages: preprocessing (modeling), evaluation and simulation, and post-processing (visualization). In the first stage, the FE model is defined by creating an input file, which involves an engineer's design for the following stage. Next, the FE model is processed, and then produces an output visual file. Then, the post-processing stage is a visual rendering stage and it generates various types of data such as a report, an image, an animation, etc. from the output file [55].

The most critical and time-consuming stage is the preprocessing stage where the material properties, geometries, loading and boundary conditions are specified. Initially, the preprocessing stage started by generating the two-dimensional (2D) quadrilateral surface mesh elements in MIMICS by Materialize. The 2D surface was then converted to a volumetric three-dimensional solid meshed part that is described by a continuum threedimensional (3D) meshed volume C3D. The most advantageous 3D FE domains are tetrahedral (tets), pentahedral (pies) and hexahedral (bricks), with straight or curved edges and flat or non-flat faces as shown in figure 18. Both eight-node Hexahedral finite element 
type, also known as C3D8 and the tetrahedral element may come as 4-noded (C3D4) or 10-noded (C3D10) are shown in figure 19.
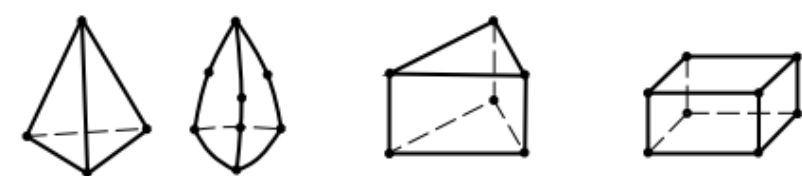

Figure 18: 3D FE domains of tetrahedral and hexahedral elements [58]
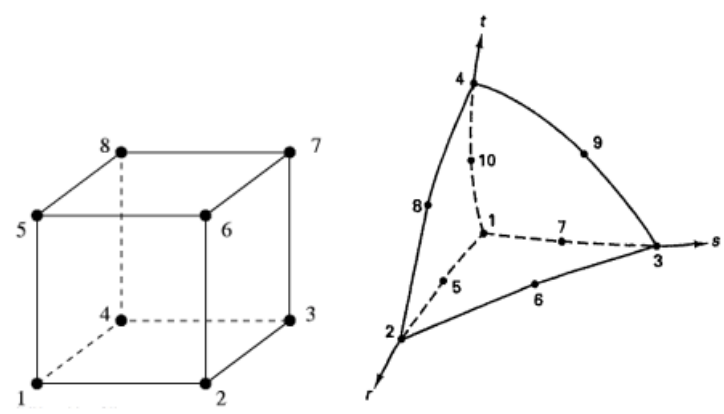

Figure 19: Hexahedral element (left); Tetrahedral element (right) [60]

Each element has one node at each corner, and each node has six degrees of freedom. These nodes can move horizontally (x-direction), vertically (y-direction), and translationally (z-direction), as well as rotationally about the $\mathrm{x}, \mathrm{y}$, and $\mathrm{z}$-planes. A mathematical matrix acts as an identifier of each element as well as representation of the interaction among the degrees of freedom of a set of nodes. This matrix leads to determination of the structure's unknown forces and displacements, and it is called stiffness or coefficient matrix. The 3D tetrahedral element type was selected for the current project as the generated tetrahedral volume mesh is approximately similar to the actual bone tissue structure.

Identifying element features was the following step of element type selection. These features include the material properties and real constants such as modulus of elasticity, Poisson's ratio, density, etc. Nodes' positions and their respective displacements are defined in a matrix form as a function of its coordinates. A function of nodes' coordinates is expressed in a matrix form to define the nodes' positions and their respective 
displacements that are used by ABAQUS. Relationships between displacements, stresses, and strain values are represented by the basics of mechanics used in the matrix. The material property used in the analysis for the bone tissue was specified as constant isotropic. Therefore, no real constants were required for the chosen specific element type.

ABAQUS users should pay close attention when they apply loading and boundary conditions in order to end up with a solvable model. For instance, some nodes are required to be fixed so they could converge on a unique mathematical solution as well as the model is sufficiently defined. The purpose of applying boundary conditions is to restrain one node or more to move in one or more of the six degrees of freedom.

In the processing stage, ABAQUS runs the model, and then determine the degree of freedom values. Within each element, values of degree of freedom are mapped to points by a mathematical function known as the element shape function. Thus, assumed behavior of a selected element is represented by its element shape function. Therefore, the accuracy of the FEA model is directly depending on excellent correlation between the assumed element-shape function and the true behavior of the system. Moreover, each local stiffness matrix is derived by its respective element, and is combined to develop an overall stiffness matrix as a whole for the FEM. Basically each element's matrix is solved to determine degree of freedom values for each node, then it is substituted for the solution of the whole model. The governing equation for a structure:

$$
[\mathrm{K}](\mathrm{D})=(\mathrm{R})_{\mathrm{F},}[61]
$$

where $[K]$ is the global stiffness, (D) is the vector of nodal displacements, and (R) is the vector of forces applied upon the nodes. Each degree of freedom of a particular node is represented by a respective component of the vector (D). The same is correct with the 
vector $(\mathrm{R})$. Several mathematical operations are required in order to enhance attaining equilibrium equations, and thus finding the FE solution. Minimization of potential energy is one of those mathematical operations so the equilibrium state for structural problems is achievable. An equilibrium formula is developed if the first derivative of the potential energy with respect to displacement is equated to zero. Strain energy, of an elastic body, is subject to some loss due to displacement caused by body forces, surface tractions, and nodal forces. The remaining energy after loss is the potential energy as shown in the following equation:

$$
U_{0}=\frac{1}{2}(\epsilon)^{T}[E]\{\epsilon\}-\{\epsilon\}^{T}[E]\left\{\epsilon_{0}\right\}+\{\epsilon\}^{T}\left(\sigma_{0}\right),[61]
$$

where $U_{0}$ is the strain energy per unit volume, $\epsilon$ is the strain, $\mathrm{E}$ is the elasticity tensor of the material, $\epsilon_{0}$ is the initial strain, and $\sigma_{0}$ is the initial stress. The total strain energy is as follows:

$$
\Pi_{\mathrm{P}}=\int_{\vee} U_{0} d \vee-\int_{\vee}\{f\}^{T}\{F\} d \vee-\int_{V}\{f\}^{T}\{\Phi\} d s-\{D\}^{T}(P),[61]
$$

where $\Pi_{P}$ is the potential energy, (f) are the displacements of arbitrary points in the body, (F) are the body forces, $\{\Phi\}$ are the surface tractions, (D) are the nodal displacements, and (P) are the nodal forces. The equation of equilibrium is resulted from equating the differentiation of $\Pi_{P}$ to zero.

If an FEA model is meshed during the preprocessing stage, the selected mesh density should be appropriate. Otherwise, the measurements may be adversely impacted by resulted discontinuities. An FEA model becomes valid once it has run successfully as well as resulted output database are similar to experimental results. Consequently, some 
input data such as the values of boundary conditions, material properties, displacements, or load can be infinitely modified to represent other FEM with the same structure.

\subsection{Recent Applications of FEA:}

Many studies conducted over different human skeletal sites revealed fascinating results when HRCT was combined with FEA [22, 40, 62-64]. Rietbergen et al. (1999) demonstrated the ability of 3D structural indices to reflect mechanical aspects of trabecular bone [40]. The Young's moduli were efficiently calculated when the HRCT was combined with the FEA. The linear multivariate regression analysis was used to correlate between structural indices and elastic constants. A better correlation was observed when one of the 3D structural indices was included as independent variable than when the volume fraction of trabecular bone was the only independent variable [40].

Van Rietbergen et al (1999) calculated structural indices and elastic constants of human cancellous bone from different skeletal sites and investigated the predictive value of different 3D structural indices for the elastic properties of bone [40]. The skeletal sites included the iliac crest, lumbar spine, femoral head, and calcaneus from which a total of 237 specimens of cancellous bone were taken and imaged. They found better correlations when they included more than one of the 3D structural indices as independent variables, which turned out to $\mathrm{R}^{2}$ of $92 \%$. Consequently, the prediction of elastic stiffness constants for cancellous bone samples was clearly improved when volume fraction was supplemented with 3D structural indices [40].

Pistoia et al (2003) evaluated which structural parameters (other than bone mass) were the best predictors for changes in bone mechanical properties of the human radius. Eight different FE models were developed based on scanning the same human radius to 
simulate various bone atrophy scenarios, and then to investigate whether the original strength of the affected bone can be recovered if bone loss is restored by thickening of the remaining structures. The failure criterion was assumed based on earlier study such that the failure would be initiated as soon as $2 \%$ of the bone tissue is strained in excess of 7000 microstrain. Results revealed that the original bone strength was not recovered when the bone mass was restored. The study demonstrated that the importance of different parameters for the prediction of bone strength also depends on the mechanical loading [62].

Keaveny et al. (2007) simulated a removal of the shell from the vertebral body, and then presented the associated mechanical effects in terms of overall change in vertebral structural stiffness and of the tissue level stresses. They concluded that the thin vertebral cortical shell can remarkably carry significant load by virtue of representing a large proportion of the vertically aligned bone tissue within the vertebra. Additionally, the shell also maximizes the load carrying capacity of the trabecular centrum, particularly around the periphery [63].

Keaveny et al. (2015) performed HRCT-based, linear elastic FEA, which was validated in a previous study, to investigate the relative role of the cortical vs trabecular bone in the load-carrying capacity of the proximal femur. They specifically quantified the fraction of frontal-plane bending moment shared by the cortical vs trabecular bone in the femoral neck as well as the associated spatial distributions of stress. Satisfactory results revealed that in both loading modes, the trabecular bone took most of the load at the most proximal region in the femur neck, whereas at the most distal region the cortical bone took most of the load [64]. 


\section{CHAPTER 3: METHODS}

\subsection{Overview:}

This chapter discusses three major operations: high resolution scanning and multimodality image processing of a representative cadaveric human proximal femur; experimental testing to simulate and re-create the fracture event which is caused by a fallto-the-side mechanism in the elderly; and computational modeling simulation of hip fracture caused by fall-to-the-side loading by employing finite element analysis. The computational modeling was used to simulate the original experimental testing by creating an accurate model of the cadaver femur, and then performing a parametric analysis in which the morphological parameters of femoral neck cortical and trabecular thickness were varied to determine the effect on proximal femur fracture strength.

Detailed explanation of each operation is highlighted throughout this chapter. The next section, 3.2: Scanning, begins with a brief introduction for the human femur used in this research. The following subsections describe the scanning protocol, which starts from determining input data related to the femur positioning, such as scanning resolution, and ends with generating the reconstructed images. Section 3.3 describes the entire procedures for processing grayscale images using Fiji-ImageJ_1.53c, and how they were converted to binary images that were visually representative of the basic model. This section also articulates the morphological operations, erosion (thinning) and dilation (thickening), performed upon the basic binary images. Section 3.4 explains the generation of the 3D surface part and how it was optimized in order to obtain an exportable model. The 
processed model was built with the 3D triangulated surface having zero overlapping and zero intersecting triangles using Mimics/3-Matic software (Materialize, Leuven, Belgium). Section 3.5 starts with conversion of the triangles to a 3D solid tetrahedral mesh elements part using FE methods through ABAQUS software (Simulia Inc., Waltham, MA). In this program, multiple modules were developed to simulate the cadaveric bone behavior in the experimental fracture test. This section ends with presenting both the binary images created in ImageJ and their final conversion to $3 \mathrm{D}$ solid tetrahedral parts in ABAQUS. Section 3.6 describes how the experimental modeling analyses were set-up, executed, and visualized and tabulated for reporting results. Section 3.7 explains the validation methodology for the basic FE model. To validate the basic model, the strain gauge strains were compared to the calculated strains from the basic model at the same location as the gauges. The determination of failure criterion is explained separately in section 3.8 because model failure behavior is not obvious when only linear elastic material properties are used. Finally, Section 3.9 describes how the eight different parametrically varied morphological models were executed and interpreted to determine the effects of cortical and trabecular thickening and thinning on calculated fracture strength.

\subsection{Scanning:}

\subsubsection{Human Model:}

Both proximal femurs were extracted from a sixty-eight-year-old female human cadaver at the Fresh Tissue Dissection Facility, University of Louisville. The current study used the right proximal femur. The general guidelines of cleaning bones include removing adhering soft tissues by dissection or scraping with sharp instruments and 
taking care not to introduce damage to the bone tissue itself. A 50\% solution of alcohol was used to further clean and degrease the bone surfaces. The specimen was stored in the lab's freezer at $-20 \mathrm{deg} \mathrm{C}$ after cleaning. To avoid deterioration of properties, the bone was kept moist by wrapping in saline soaked gauze before freezing.

\subsubsection{Scanner Setup:}

The micro-CT imaging was performed at the University of Louisville Orthopedic Bioengineering Lab with a high-resolution scanner (Actis HR225-150; BIR, Lincolnshire, IL, USA), as fig(20) shows the main components of a typical HRCT scanner. In order to obtain high resolution or micro-computed tomography (micro-CT) images with high quality, the scanner was prepared for scanning using standard startup procedures to assure consistency and repeatability. The setup process involves critical steps such as central ray correction and calibration of pixel size.

Scanning/imaging:

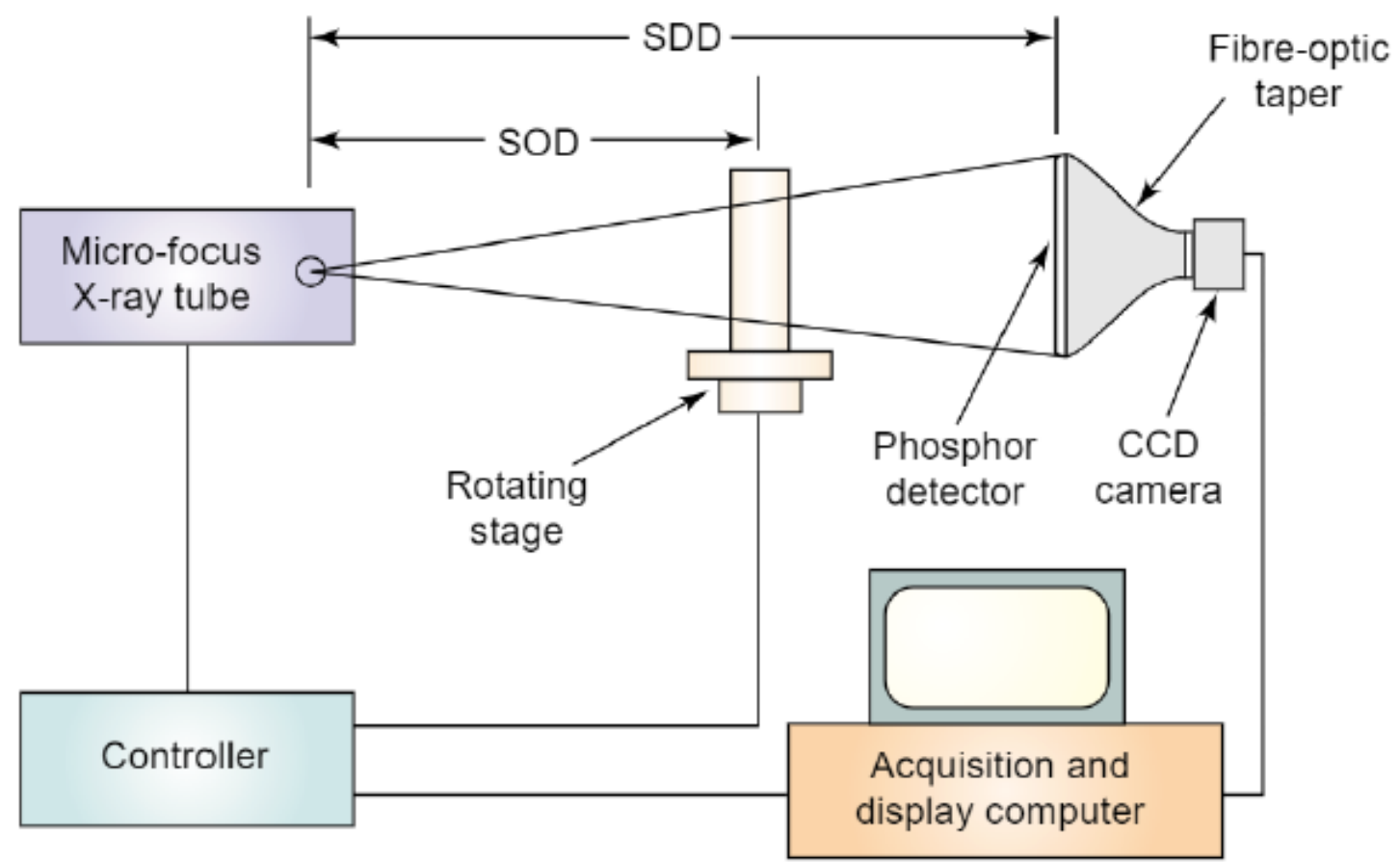

Figure 20: An in-vitro microscopic computed tomography (Micro-CT) scanner. A specimen, mounted on a rotating stage, is positioned between an X-ray source and detector. The source-to-object distance (SOD) and 
source-to-detector distance (SDD) are selected to provide the appropriate amount of geometric magnification. Typically, the SDD is $\sim 20 \mathrm{~cm}$, and the SOD ranges between 7 and $18 \mathrm{~cm}$. X-ray projections are acquired by a phosphor detector, coupled to a CCD camera by a fibre-optic taper, which reduces the size of the image. During acquisition, the computer controls the $X$-ray tube and specimen stage, obtaining $\mathrm{X}$-ray projections at hundreds of angular positions [65]

A specimen, mounted on a rotating stage, is positioned between an X-ray source and detector. The source-to-object distance (SOD) and source-to detector distance (SDD) are selected to provide the appropriate amount of geometric magnification, $\mathrm{M}$ as the following formula shows the relationship between them.

$$
M=\frac{S D D}{S O D}
$$

X-ray projections are acquired by a phosphor detector, coupled to a CCD camera by a fiber-optic taper, which reduces the size of the image. During acquisition, the computer controls the X-ray tube and specimen stage, obtaining X-ray projections at hundreds of angular positions [65].

The right femur was scanned at an isotropic voxel resolution of $60 \mu \mathrm{m}$. The femur was placed in a cylindrical plastic tube that was mounted on the scanner's gantry. Cloth padding was added surrounding the femur in the tube to prevent the femur from movement during scanning rotations. An approximate length of the femur of $200 \mathrm{~mm}$ was imaged including the entire femoral head and extending distally to the level of the lesser trochanter. A total of 32 rotations were needed to scan the proximal femur, covering $6.42 \mathrm{~mm}$ for each scan increment. The resulting $6.42 / .06$ x $32=3,424 \mathrm{raw}$ files each contained a single row image repeated at every angular increment of the scan for each "slice" of the overall scan. The scanner software then generated a stack of two-dimensional images by reconstructing the raw files into multiple image slice files (2048 x 2048 pixels) that could be assembled into image stacks representing crosssectional images of the proximal femur. 


\subsection{Image Editing and Segmentation:}

Prior to three-dimensional analysis, the individual image slice files had to be processed to isolate the bone tissue from the soft tissue, marrow, and other grayscale data that did not represent bone substance.

The first step, crop processing, was executed on the tiff images using Image-J software (Wayne Rasband, NIH, Bethesda, MD). Image cropping is a tool used to trim or completely to eliminate the edges of an image. Edges trimming/elimination is beneficial in reducing the time elapsed in the subsequent image processing. In other words, pixels are removed from an image when the image is cropped. The size of all images was reduced from $2048 \times 2048$ pixels to $1460 \times 815$ pixels as illustrated in the following figure.

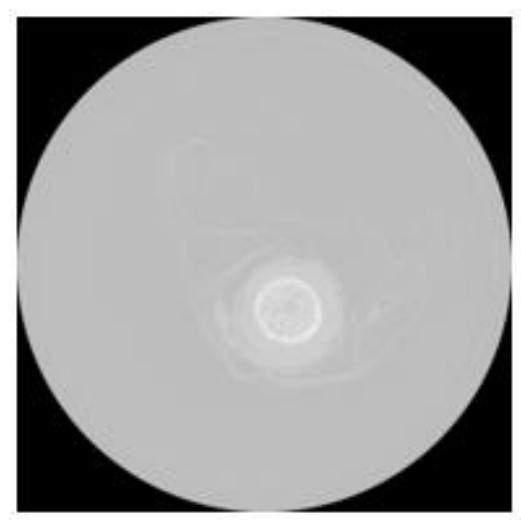

Figure 21: The original gray image (left) and the cropped image (right) in ImageJ

An auto adjustment of brightness and contrast was performed in order to easily distinguish between the bone boundaries and the surrounding. For example, optimizing the brightness and contrast enhance well in tracing the bone outer boundary. 


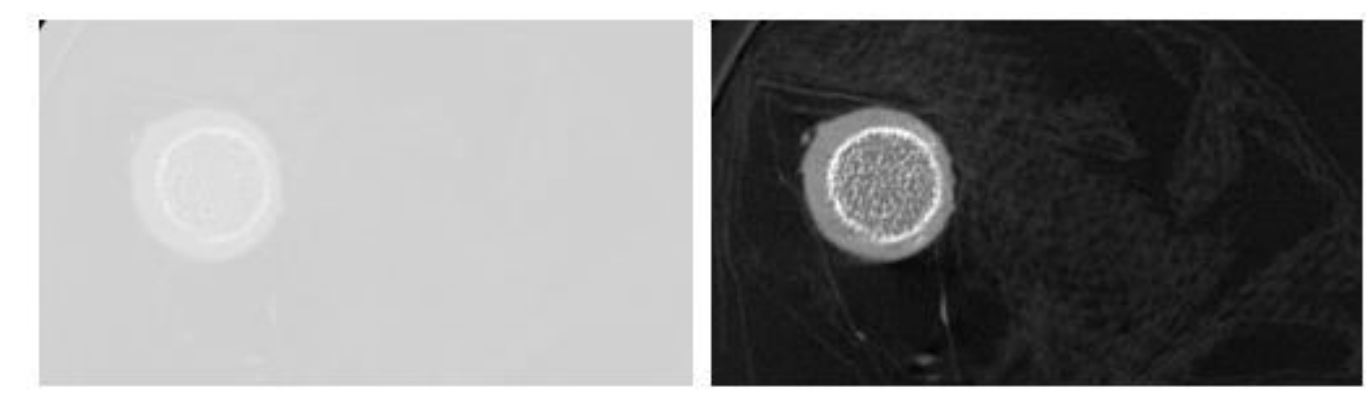

Figure 22: Using brightness and contrast tool in imageJ to make the original gray image (left) looks

brighter image (right)

After cropping, all volumes other than the volume of interest (VOI) were converted to black color, which was accomplished using the MIPAV (Medical Image Processing, Analysis, and Visualization) application and performed in three steps. In the first step, the "Live Wire VOI " selection tool was used incrementally on one image out of every ten images in the stack in order to trace the outer boundary of the femur more efficiently without having to process every image. Some images include two separate regions of interest at the trochanter and the femur head, so the double selection was used simultaneously as shown in the following image.

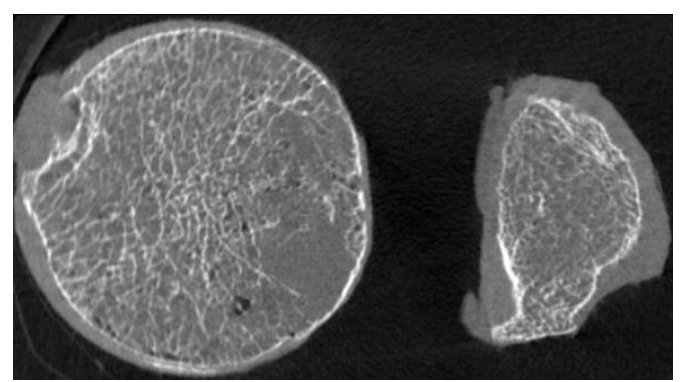

Figure 23: Double selection was used in MIPAV for images which has two separate regions of interest

Then interpolations were processed between every two consecutive VOI selections, which were each ten slices apart, by the "interpolate" command to automatically generate "Live Wire" selections for the entire image stack. In the second step, the entire stack was selected to convert all VOI to binary masks with both solid white color (8-bit gray level: 255) for the bone tissue and solid black color (8-bit gray 
level: 0) for the non-bone areas. A "mask" is a continuous region of the image that is selected and assigned to have a specific gray level. In the third step, the auto adjusted gray images were multiplied by the mask images, and hence a new modified stack of gray images was obtained as shown in the following figure.
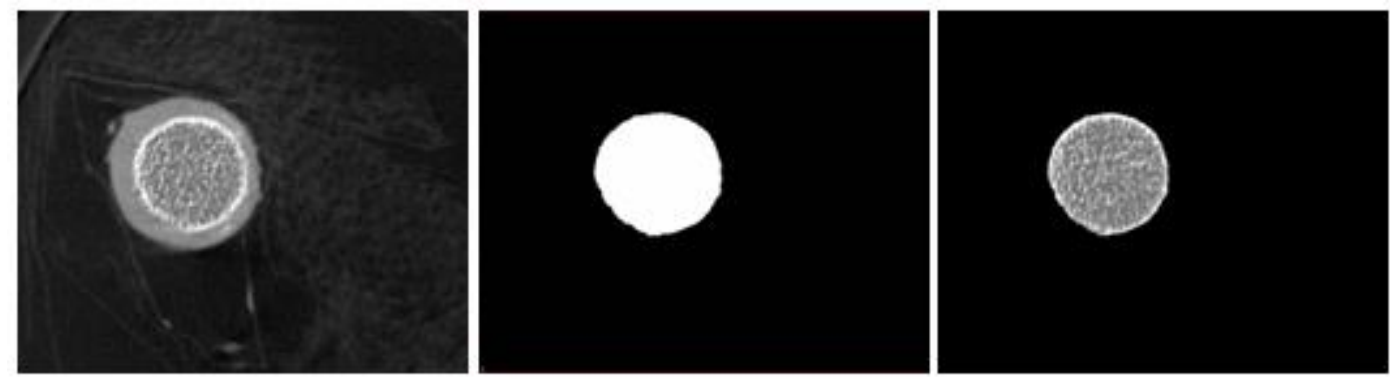

Figure 24: The mask image (middle) was created from the original image (left). The right image is the product of the left image and the middle image in ImageJ

Because partial volume effects, which are common in computed tomography images, cause thinner structures to be represented by darker gray values than thicker structures, it was necessary to first process the cancellous bone and the cortical bone separately. Therefore, the cortical bone was separated from the cancellous bone based on semi-automatically identified and modified volumes of interest (VOIs). The "live wire" tool was used incrementally upon one image of every ten images in order to delineate the internal boundary of the cortical bone. However, some discontinuities were present over the cortical bone, so the "polyline" tool was used to manually tracing the cortical bone boundary. The selections were interpolated for the rest of the images, and then 2D masks with fully solid white color were created for the cancellous bone regions that were located inside the VOIs. Next, the "image calculator" utility was executed several times for multiplication, addition, and subtraction operations until two separate stacks of cortical bone and trabecular bone tiff images were obtained. The following figure shows a sample of the separation process. 


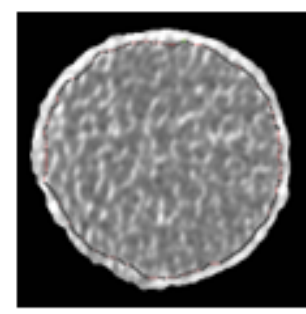

(a)

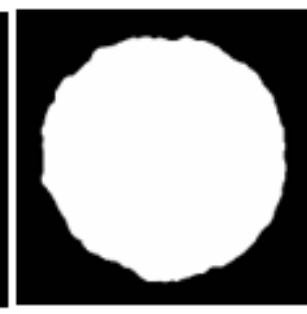

(b)

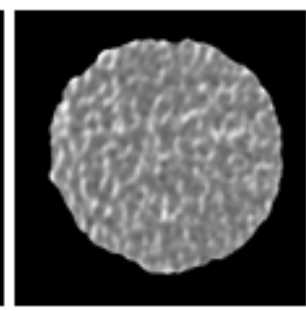

(c)

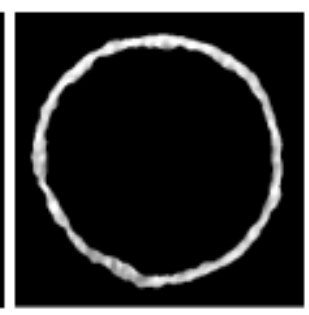

(d)

Figure 25: The original image (a), A created mask for the cancellous (b), A separated cancellous image (c), and the separated cortical bone (d) in ImageJ

In order to avoid any potential errors caused by gaps or disconnection between the cortical and cancellous regions, the mask of cancellous bone was dilated. Hence, at least four pixels from the external boundary of the cancellous should overlap with four pixels from the internal boundary of the cortex.

The two stacks of gray tiff images for cortical and cancellous bone were imported into Image-J in order to obtain a stack of binary images for the basic femur bone. The process started with multiple automatic adjustments of brightness and contrast as illustrated in the following figure.
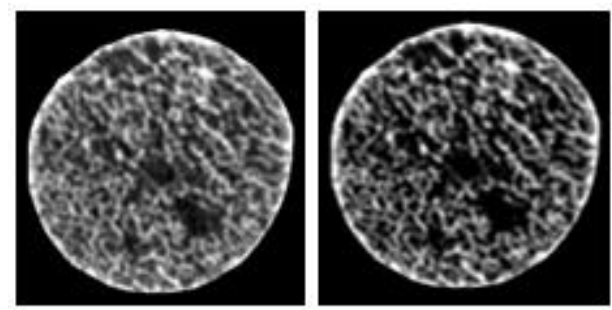

Figure 26: A brightness and contrast performed on the left image to get the right image in ImageJ

The edges of the new adjusted images were filtered by using the tool called "Unsharp Mask". This tool enhances and sharpens edges by subtracting the blurred regions from the original image. It also rescales the image to obtain the same contrast of large (low frequency) structures as in the input image. The two input parameters called Radius and Mask Weight were set by trial and error until the best representation was obtained visually as shown in the following figure. 


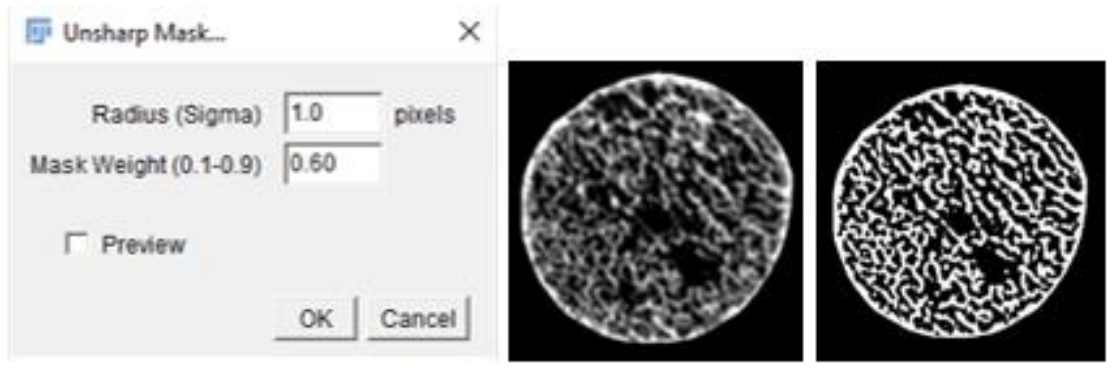

Figure 27: The blurred regions were removed from the left image in ImageJ to obtain the right image

An additional adjustment process was performed over the un-sharp mask images by the "Threshold" tool as displayed in the following figure.
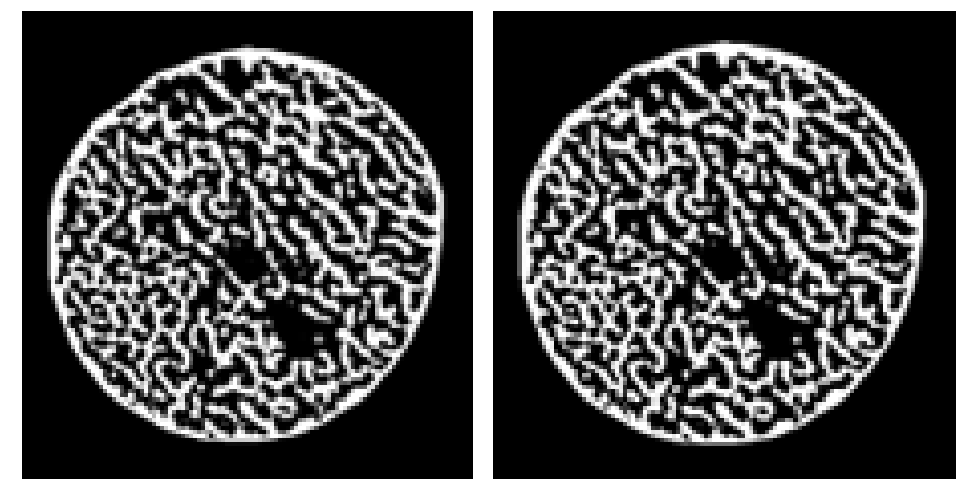

Figure 28: An automatic threshold was applied over the left image in ImageJ to obtain the right image, as one of enhancement steps to achieve binary images

Thresholding is a technique for dividing an image into two (or more) classes of pixels, which are typically called "foreground" and "background". In this picture for example, the white color was selected for the foreground, whereas the black color was selected for the background.

When converting image stacks into finite element models, it was important that the three-dimensional structures that were created did not have any unconnected regions. If these existed, they would not have been subjected to the boundary conditions or the applied loads in the analysis. This would have left sections of the model unconstrained and they could have experienced infinite or near infinite displacements which would have resulted in execution errors. Therefore, the connected 
regions were highlighted by a Plugins tool called "Find Connected Regions". This plugin highlights all of the connected regions with the same value (where that value is greater than 0 ) in a 8 bit label field. The process was done by a point selection over an area of interest as illustrated in the following figure.
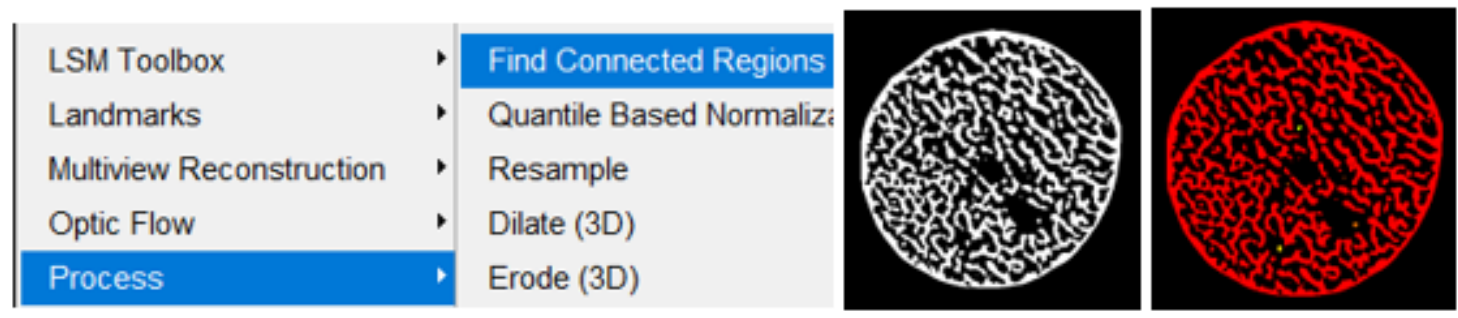

Figure 29: The unconnected regions were removed from the left image to get the new image in the right in ImageJ

The brightness and contrast were adjusted for the connected region images, and then converted to binary images as illustrated in the following figure.
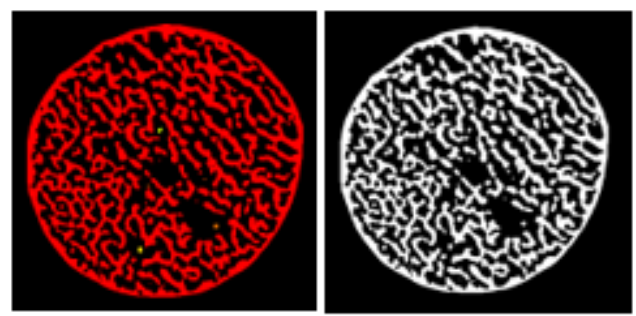

Figure 30: The original gray image of cancellous on the left converted to binary image on the right in ImageJ

In Image-J, the cortical bone images were converted from gray to binary images as shown in the following figure.
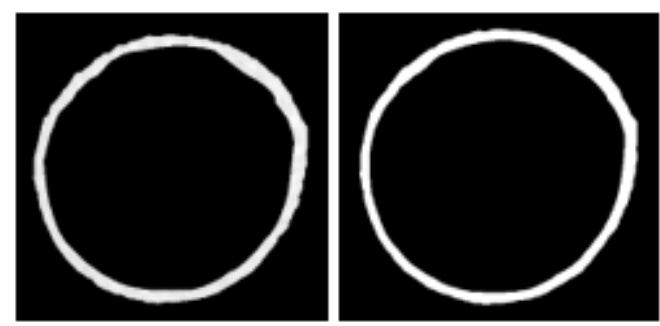

Figure 31: The original gray image of cortical bone on the left converted to binary image on the right in ImageJ 
The basic femur binary images were obtained by combination of the cortical bone binary images with the cancellous bone binary images as displayed in the following figure.
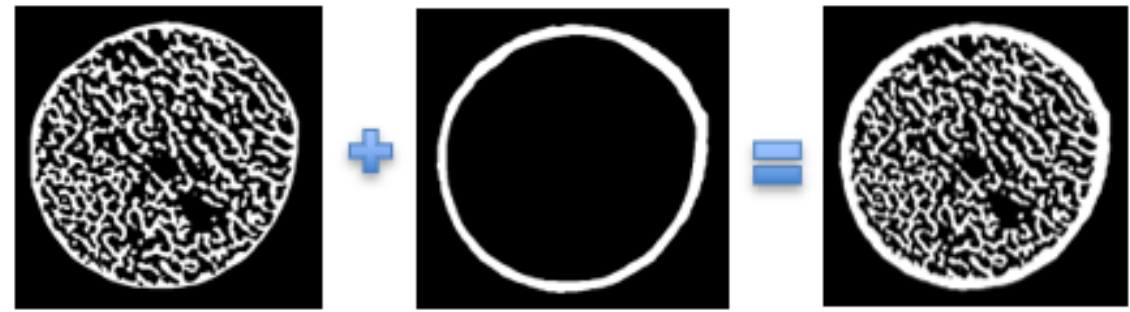

Figure 32: The combination between the binary images for cancellous and trabecular bone in ImageJ

\section{Connected Components Definition:}

The binary images for the basic model underwent various morphological operations in order to generate eight models with varied morphological parameters of dilation (thickening) or erosion (thinning) processes. One of the most important factors involved in dilation and erosion processes was the connectivity characteristics. Voxels are connected based on assigned intensity values as well as assigned connectivity path. Three types of connectivity are commonly used in the $3 \mathrm{D}$ processing such that two voxels are connected when their cubes share a common face (6-connectivity), a common edge (18-connectivity), or a common vertex (26-connectivity). In FEA, singularity connections like either edge to edge or vertex to vertex causes extremely high stress values across such connections so the 6-connectivity was selected for this project as illustrated in the following figure.

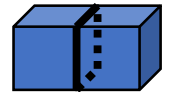

Two Connected voxels

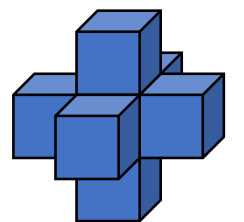

All voxels connected to the center voxel 
Erosion removes pixels from the edges of objects in a binary image, whereas Dilation adds pixels from the edges of objects in a binary image. Further options should be specified as shown in the following image:

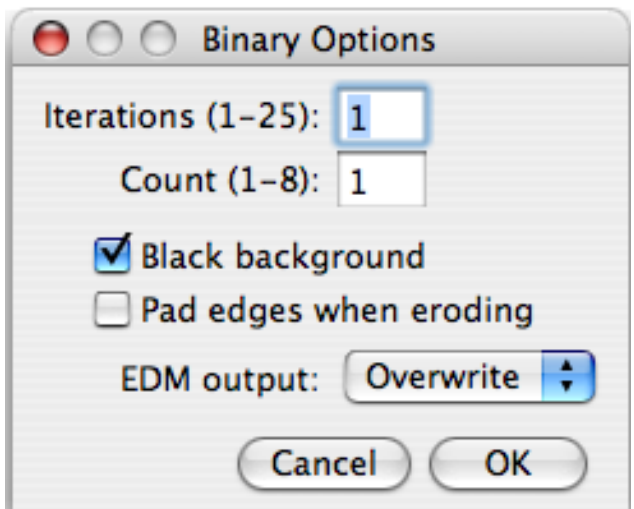

Figure 33: Binary options in ImageJ for erosion or dilation processes. "Iterations" specify number of times for a process and "Counts" specifies number of adjacent pixels in background necessary before a process

Iterations specifies the number of times erosion, dilation, opening, and closing are performed. Count specifies the number of adjacent background pixels necessary before a pixel is removed from the edge of an object during erosion and the number of adjacent foreground pixels necessary before a pixel is added to the edge of an object during dilation. The dilation and erosion processes were 3-dimentionally applied over the binary images for the basic model to generate eight stacks of binary images with varied morphological parameters using ImageJ. Figure 34 shows a trabeculae 3dimentionally eroded (removing) and dilated (adding) total of $120 \mu \mathrm{m}$ in each direction, vertical and horizontal but not diagonally to insure face-to-face connectivity. 

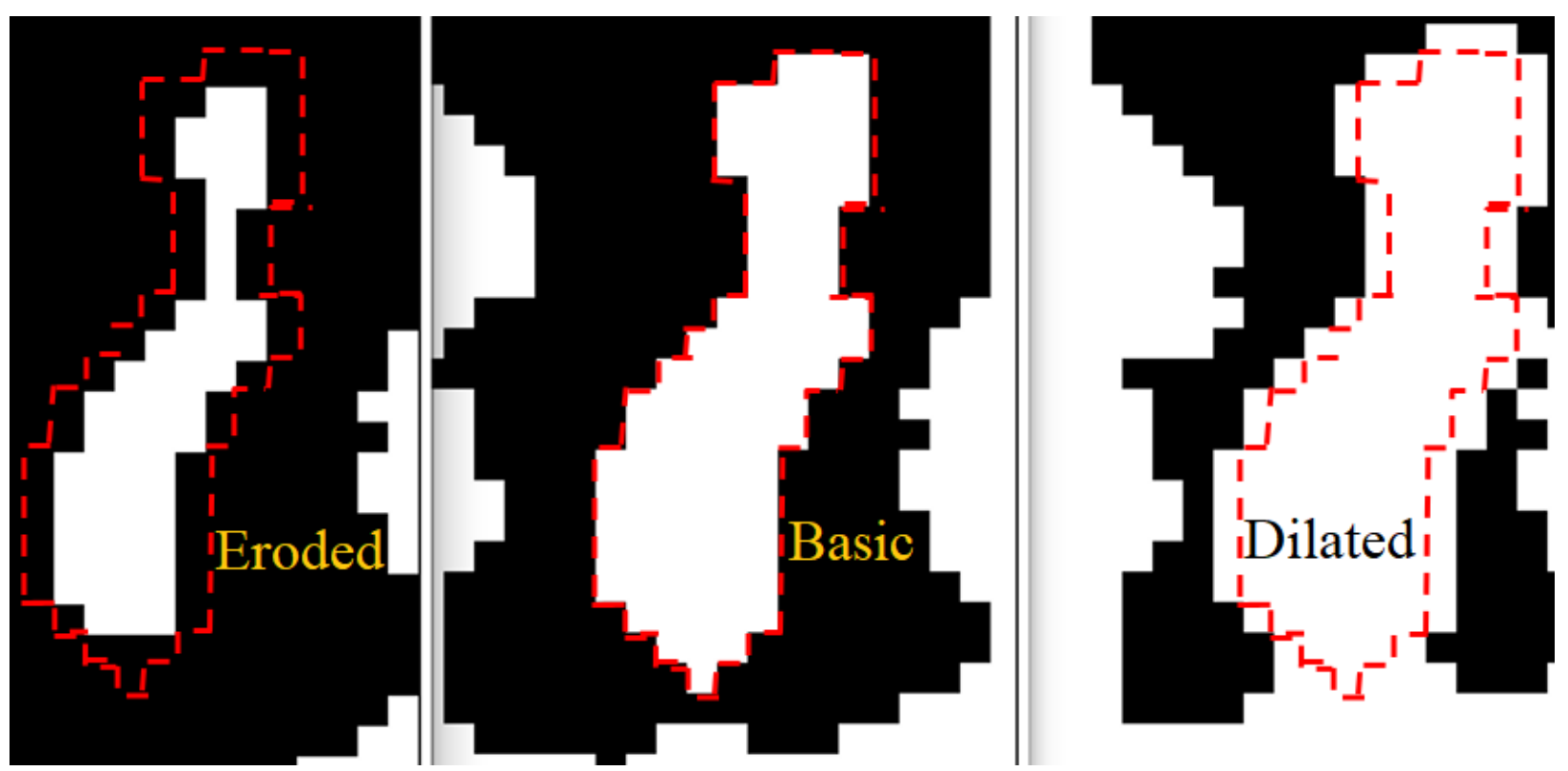

Figure 34: The binary image for the basic model in the middle was 3-dimentionally eroded in the left and dilated in the right using ImageJ

\subsection{MIMICS/3-Matic Image Stack Processing:}

The new stacks of the binary tiff images for the femur models were imported into Materialise Interactive Medical Image Control System (MIMICS, Materialise, Lueven, Belgium) program in order to convert the entire stack into 3D triangulated surface part. The program could not complete the conversion for limitations regarding the large number and the small size of triangles, so the resolution was reduced 50 percent by increasing the base-element dimension from 60 microns to 120 microns. Thresholding ( $)$ was used to create a first definition of the segmentation object. The object was defined based on one lower threshold or based on a lower and a higher threshold. In the current case, the segmentation object contained all pixels in the images with a value higher than or equal to the threshold value 253 for gray level 8-bit. A sample of the thresholding process is illustrated in the following figure. 

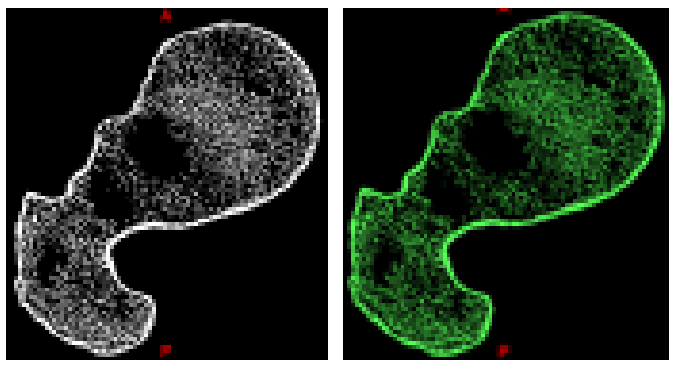

Figure 35: The left image from ImageJ with $60 \mu \mathrm{m}$ was imported into Mimics, from which a new binary mask was generated as illustrated in the green image using Mimics

Following the Thresholding, the tool called "Region Growing from the "segment" menu. Within the source mask, this tool created a new mask, with different color, containing only those highlighted voxels connected to the seed point that was identified. In other words, all points in the current segmentation object connected to the marked point were moved to the target mask.

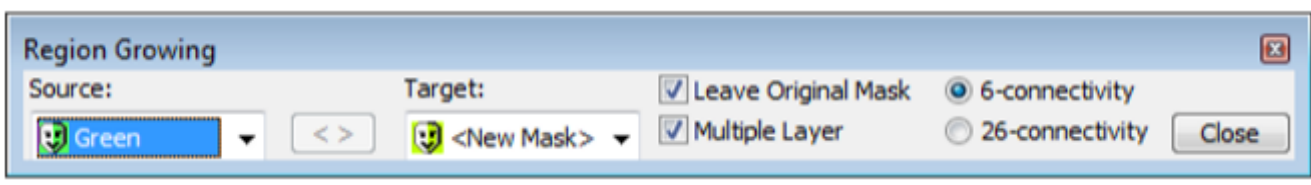

Figure 36: Region growing tool in MIMICS to create a new mask for connected regions

Source: The original mask

Target: The target mask can be a new mask or an existing object, in which case the selected region will be added to this object.

Multiple layer: The operation can be performed on one single slice (multiple layer is Off) or in 3D on all slices (Multiple layer is On): to do this, turn Multiple Layer On or Off in the Region Growing Properties toolbar.

The following figure shows the new mask obtained from region growing process. 


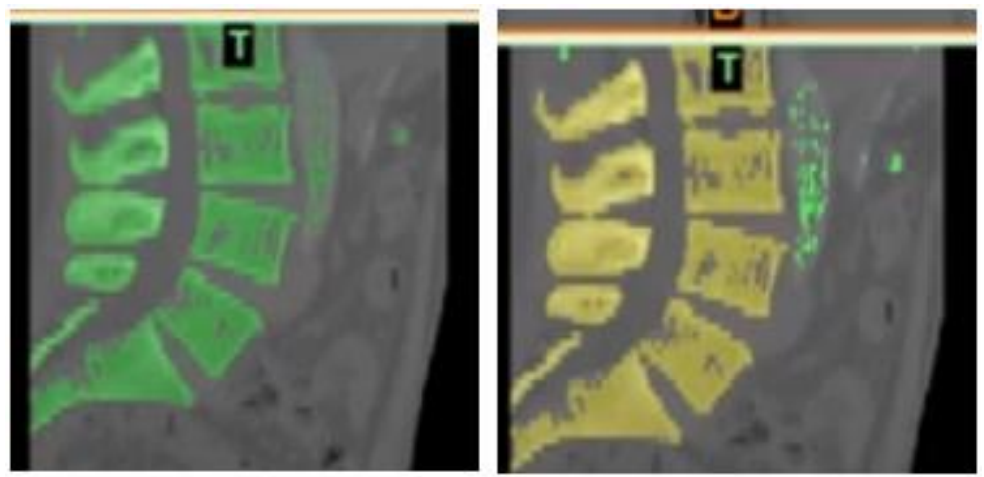

Figure 37: A region growing operation was performed over the left image to get the right image in Mimics. As noticed in the right image where the unconnected region with the green color was excluded from the new mask with the yellow color [52]

The version of Mimics used for the current project was Mimics-15, which does not have the options of connectivity method (6-connectivity or 18-connectivity). The built-in connectivity method in Mimics-15 is 26-connectivity, which includes vertexvertex and edge-edge connectivity. Even though, this connectivity may cause some error results due to stress concentration at vertex-vertex or edge-edge, the errors were relatively acceptable since the obtained results agreed well with results reported in previous studies.

From the segmented mask created from region growing, a 3D triangulated surface part was generated using "Calculate-3D" tool from the segmentation toolbar, in which the resolution was reduced from $60 \mu \mathrm{m}$ to $120 \mu \mathrm{m}$ for the size limitation in MIMICS. Once the 3D surface part was created, it was exported into 3Matic using "remesh" command where the process of meshing optimization wizard was performed. That process is required for models targeted to be converted to solid tetrahedral element mesh parts. The 3D part created in MIMICS and exported into 3Matic is illustrated in the following figure. 


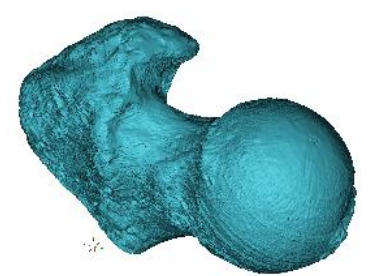

Figure 38: The calculated 3D surface part in Mimics was transferred into 3-Matic for re-mesh optimization process

During the meshing wizard, several factors were repeatedly checked and fixed such as triangular overlapping, triangular intersecting, or holes. When the desired mesh quality with no such meshing errors that potentially influencing successful execution of FE modeling was obtained, the input file was exported into ABAQUS with the 3D triangulated mesh surface as illustrated in the following picture.

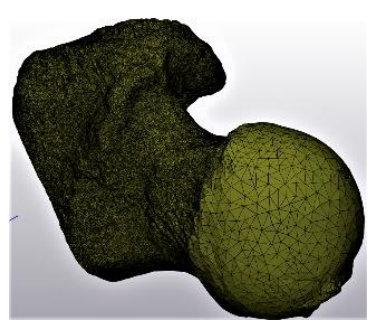

Figure 39: The 3D surface meshing optimization completed in 3Matic

In this way the complete three-dimensional geometry of each of the nine finite element models was moved from the imaging operation to the computational modeling operation, which will be described in detail in Section 3.6.

\subsection{Experimental Modeling:}

\subsubsection{Bone Cleanness and Bonding:}

The proximal femur, after having been cleaned, was embedded into a plastic pipe by using an epoxy resin body filler (Bondo, 3M Corp, Minneapolis, MN) (Figure 40). The pipe allowed the bone to be held firmly in a pivoting clamp such that the axis of the femur shaft and the femur neck could be aligned to replicate the conditions of the fall-to-the-side loading condition. 


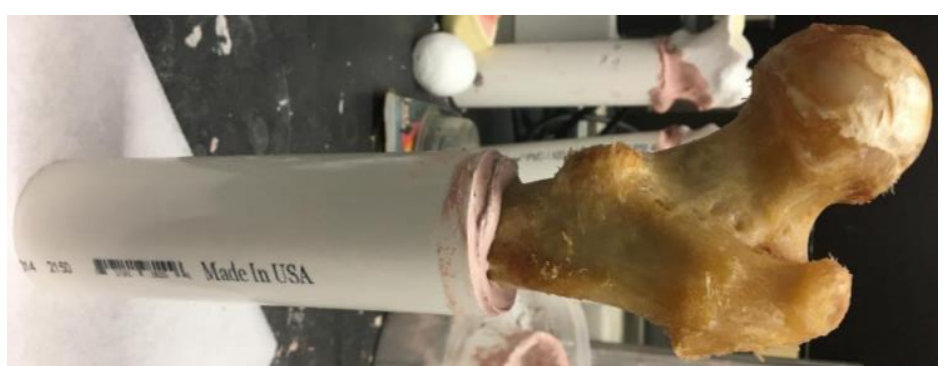

Figure 40: The cadaveric femur embedded in a plastic pipe with a Bondo body filler

\subsubsection{Strain Gauges Installation and Experiment Setup:}

Three regions on the femur surface were subjected to additional cleaning using sandpaper in order to create a clean smooth surface for mounting three strain gauges. The strain gauges with numbers 1,3 , and 4 were placed on the medial shaft below the lesser trochanter, the superolateral neck, and inferior neck respectively as shown (Figure 41). The falling to the side configuration causes the occurrence of compressive stress upon the superior neck region, whereas it causes the occurrence of tensile stress upon the inferior neck region and the medial shaft. The output from these gauges during the load to failure experiment were used to validate the basic FE model by direct comparison of the measured strains to the calculated strains at the same locations in the model.
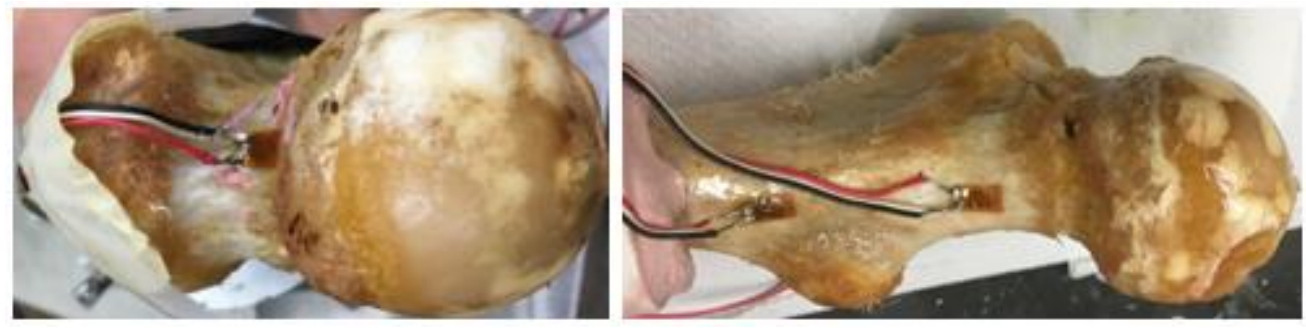

Figure 41: A strain gauge was placed on the superior neck of the cadaveric femur on the left. The other two strain gauges were placed on the inferior neck and the medial shaft

\subsubsection{Loading and Boundary Conditions:}

To represent a sideways fall configuration the femoral shaft was positioned $10^{\circ}$ from horizontal and the femoral neck axis internally rotated $15^{\circ}$ relative to a vertical axis. The fixture's cylinder, in which the femur was fixed, was allowed to rotate freely 
around the axis normal to the plane of Figure 42. The medial aspect of the femoral head was covered with a half racquetball to prevent excessively high contact stresses on the articular surface, whereas the lateral aspect of the greater trochanter was covered with a pad to prevent local crushing of the greater trochanter while also simulating the effect of soft tissue coverage. The femur was loaded to failure defined where the load deformation curve started to change from the elastic to plastic behavior (Figure 44).
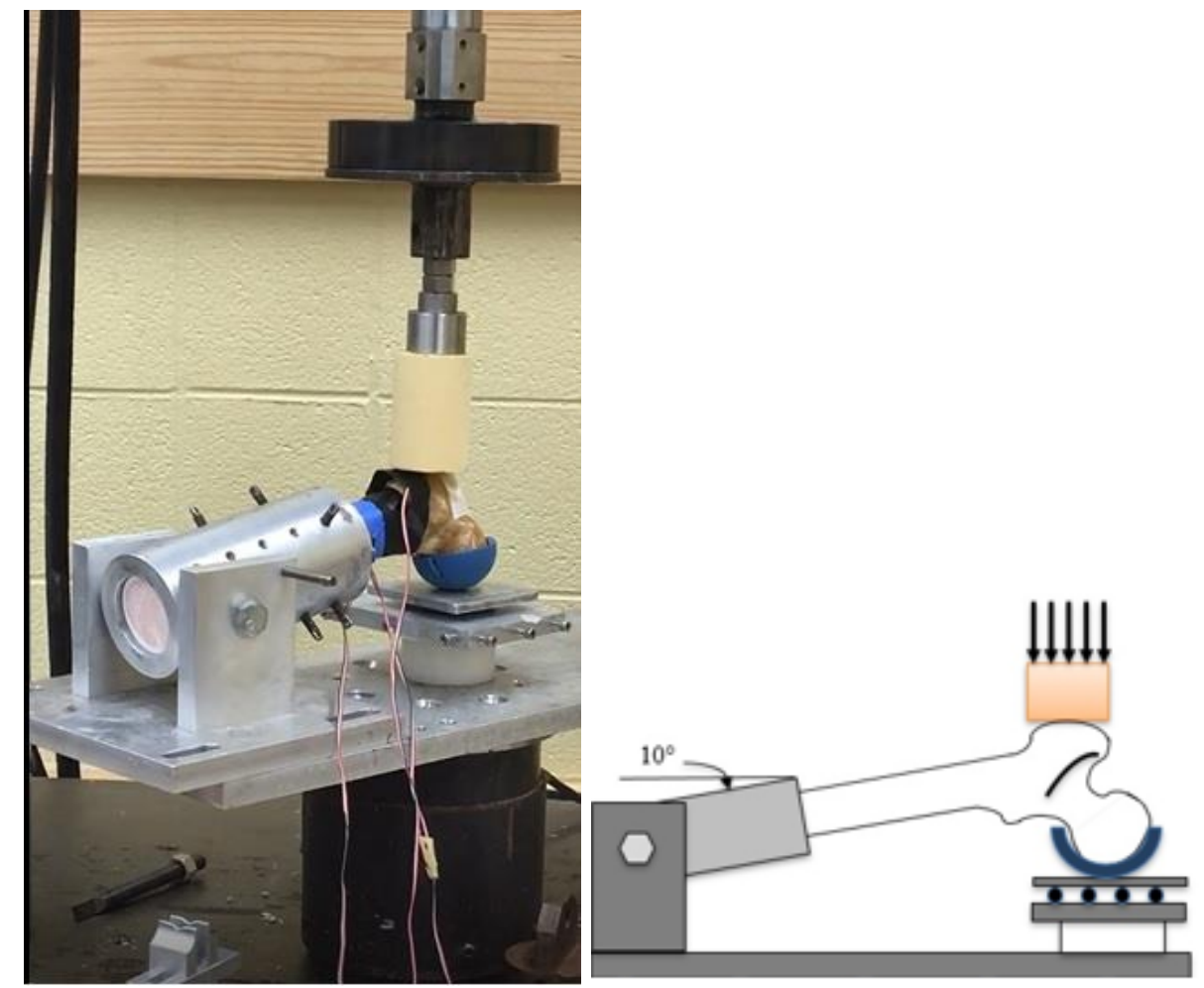

Figure 42: The setting of the biomechanical testing including loading and boundary conditions for the cadaveric proximal femur using MTS machine

\subsubsection{Strain Gauges Load Cell:}

Strain gauges were mounted according to Wheatstone half bridge configuration as illustrated in Figure 31. The Wheatstone bridge configuration is used to help measure the small variations in resistance that the sensing elements (gauge resistor wire pattern) produce corresponding to a physical change in the length of the gauge. It is the electrical equivalent of two parallel voltage divider circuits. The output of a Wheatstone bridge 
is measured between the middle nodes of the two voltage dividers. A physical phenomenon, such as a change in strain applied to a specimen or a temperature shift, changes the resistance of the sensing elements in the Wheatstone bridge. Two element legs are active in the Wheatstone half bridge.

The half-bridge type II configuration has the following characteristics:

- One active strain element was mounted in the direction of bending strain on one side of the strain specimen (top).

- Completion resistors provide half bridge completion.

- Sensitivity at $1000 \mu \varepsilon$ is $\sim 1 \mathrm{mV}_{\text {out }} / \mathrm{V}_{\mathrm{EX}}$ input.

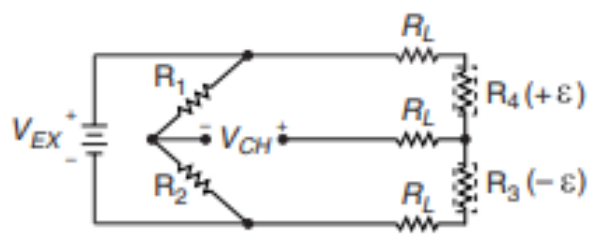

Figure 43: The Wheatstone half bridge circuit [66]

The following symbols apply to the circuit diagram and equations:

- $\mathrm{R}_{1}$ and $\mathrm{R}_{2}$ are half-bridge completion resistors.

- $R_{3}$ is the active strain-gauge element measuring strain $(\varepsilon)$ on the specimen.

- $\mathrm{R}_{4}$ is the active strain-gauge element acting as a dummy gauge to compensate for temperature.

Temperature compensation did not take place because of two reasons: the short timeframe elapsed to run the experiment and the bridges previously wired as halfbridge circuits with gauges mounted on aluminum bars, instead of bones, acting as a dummy gauge which would typically be used to compensate for temperature.

- $\mathrm{V}_{\mathrm{EX}}$ is the excitation voltage.

- $\mathrm{R}_{\mathrm{L}}$ is the lead resistance. 
- $\mathrm{V}_{\mathrm{CH}}$ is the voltage measured.

For common strain-gauge configurations where the gauge factor is 2.0, the output voltage delivered by the bridge is:

Voltage $=($ strain $\mu \varepsilon) \times($ excitation voltage $\mathrm{V}) \times\left(0.5 \mu \mathrm{V} /\left(\mathrm{V}^{*} \mu \varepsilon\right)\right)$

If the desired output is $1 \mathrm{~V}$ for $1000 \mu \varepsilon$, and the bridge excitation is $5 \mathrm{~V}$, then the bridge output is:

$\mathrm{V}_{\text {out }}=1000 \mu \varepsilon \times 5 \mathrm{~V} \times(0.5 \mu \mathrm{V} / \mathrm{V} / \mu \varepsilon)=2.5 \mathrm{mV}$

To achieve $1 \mathrm{~V}_{\text {out }}$ for each $1000 \mu \varepsilon$ produced on the gauge grid, the Gain was set at $=1 \mathrm{~V} / 2.5 \mathrm{mV}=400$.

The femur was loaded to failure defined where the load deformation curve started to change from the elastic (linear) condition to plastic (non-linear) condition. The measured raw data were load $(\mathrm{N})$ applied to the greater trochanter, displacement $(\mathrm{mm})$ of the actuator pushing down against the greater trochanter, and voltage (V) from the strain gauge circuits. Voltage is converted to strain such that $1 \mathrm{~V}$ equals $1000 \mu \varepsilon$ as determined by the settings on the Wheatstone bridge circuit. The bone behavior is expected to reveal a load-displacement curve relatively like the following figure. 


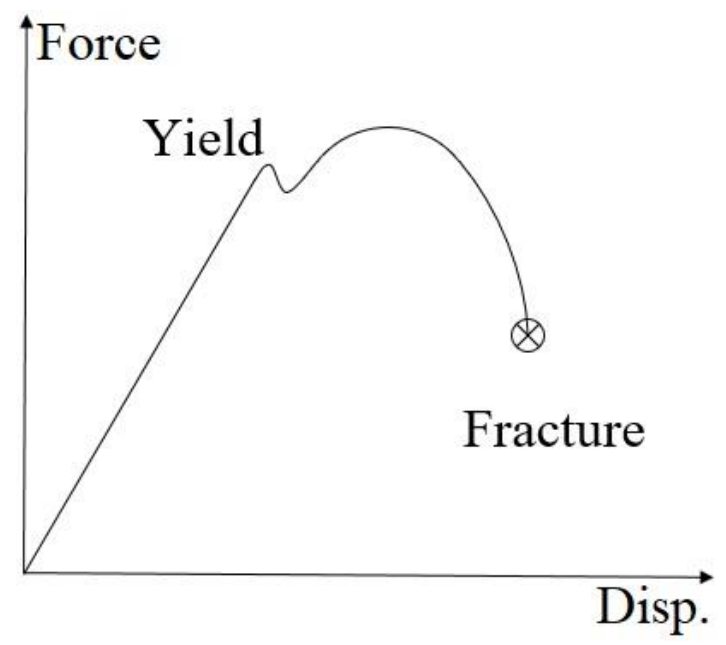

Figure 44: Exemplary force-displacement curve for a proximal femur fracture behavior. The yield region indicates to the fracture initiated, and the cross sign indicates to full breaking for a specimen

The label "Yield" denotes to the region where the femur failure initiated. The yield strain worked well in previous studies as a determinant for the failure initiation, and thus for predicting failure load [22, 48, 62, 67].

\subsection{Basic FE Model Modeling:}

\subsubsection{Basic FE Model Execution Method:}

Each model was imported into ABAQUS to simulate a fall-to-the-side loading condition with appropriate boundary and loading conditions as used in previous models and experiments. The basic model input file was developed first with the purpose of obtaining a valid FE model results that agree with the experimental data. At least two material properties, Young's modulus of elasticity and Poisson's ratio, were required in order to define the stiffness of a material and how the structure deforms when it is subjected to various loads acting on it. Young's modulus is the ratio of stress vs strain and the Poisson's ratio is the ratio of compression vs expansion of a material. All the FE models in this study were assumed to be linear-elastic-isotropic in order to relate stress to strain linearly. The first basic FE model was generated by converting the 3D 
triangulated surface mesh into a 4-noded solid tetrahedral element mesh and later converted to 10-noded tetrahedral element mesh. The generating procedure for the FE model is explained in the following steps:

- Three independent surfaces were created from manual selection from the external cortical surface at the femoral head, trochanter, and the bottom of distal part of the proximal femur.

- In the part module, four sets of nodes were created from those three selected surfaces in order to identify the loading and boundary conditions that correspond with that of the experimental fall-to-the-side loading condition (see Figures $45 \& 46)$.

- In the property module, the element material properties for the cortex and the cancellous were assigned by both modulus of elasticity (E) and the Poisson ratio (v). The modulus value was set to $10 \mathrm{GPa}$ and the Poisson's ratio value was set to 0.3 .

- Assembly module was used to create instances of all parts and to position the instances relative to each other in a global coordinate system.

- The step module was developed to create analysis step and to specify output requests. The analysis step was divided into ten-time increments. These "time increments" in a static analysis actually have nothing to do with time but serve to break the analysis up into discrete parts. In this case the entire applied load was broken up into ten equal load increments. Fields of output that were reported after each increment in separate output files by node or 
by element include, but were not limited to, Von-Mises stress, element volume, and strain energy density (SED).

- $\quad$ The loading condition was defined by applying 10,000 N spread over 500 nodes on the femur head. The load was incrementally applied over 10 "time" increments such that $1000 \mathrm{~N}$ was added with each increment.

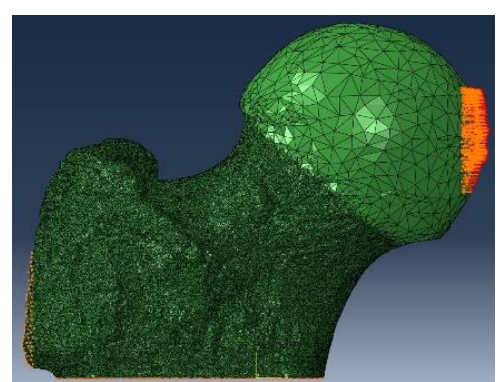

Figure 45: The loading condition applied on the femur head in ABAQUS

- Three boundary conditions were developed to simulate the boundary conditions of the experimental test where the real femur was fully broken. As illustrated in the following image, three groups of nodes are highlighted.

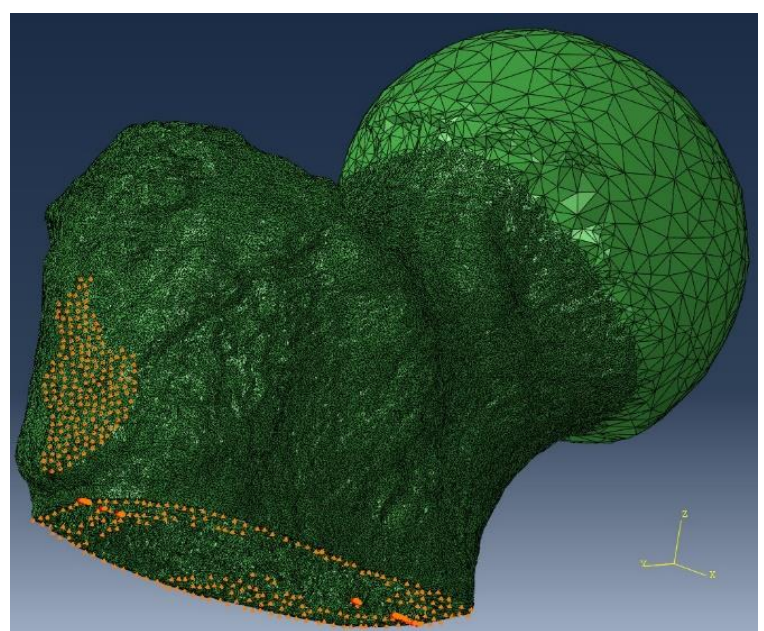

Figure 46: Boundary conditions applied on the trochanter and the bottom side of the femur in ABAQUS

The group of nodes located at the femur trochanter, lateral side of the femur, were restricted to move in the $\mathrm{x}$ direction (medial-lateral) only. The group of nodes located at the bottom side of the femur was divided to two 
groups. One group selected and aligned in the medial-lateral direction is restricted to move in the y direction (anterior-posterior), whereas the other group is restricted to move in the $\mathrm{z}$ direction (inferior-superior).

- A job module of each model was created to be submitted to Cardinal Research Cluster (CRC) for analysis. The following figure illustrates the basic model original binary image with black and white color and its conversion to the FE model with the green color:
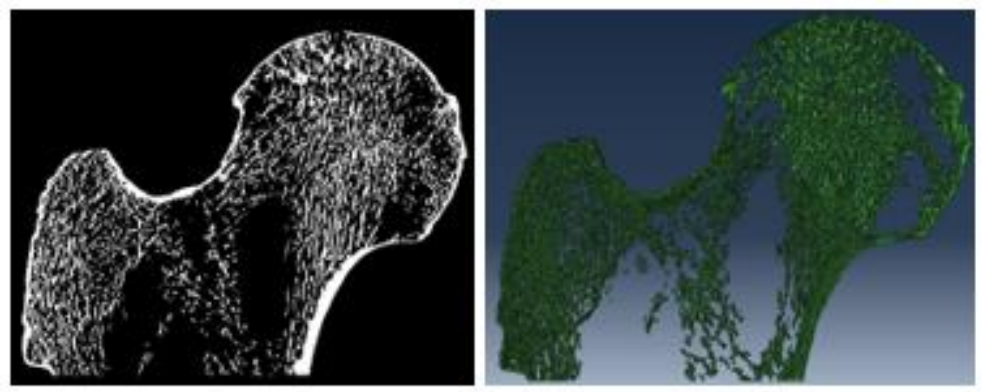

Figure 47: The left binary image in ImageJ, whereas the right image in ABAQUS. CN_TN: cortical normal _ trabecular normal, the basic model

\subsubsection{Convergence Test:}

The first task accomplished with the basic model was to demonstrate convergence of the finite element model and approach chosen for this project. The model file generated in 3Matic with the 3D triangulated surface was imported into ABAQUS and was then converted to a 3D solid tetrahedral FE mesh. The first basic FE model was generated with 4-noded elements, loading and boundary conditions applied, and the model was executed while observing the displacement of the specified node number 1128312 on the femoral head far removed from the location where the loads were applied (Figure 48). 


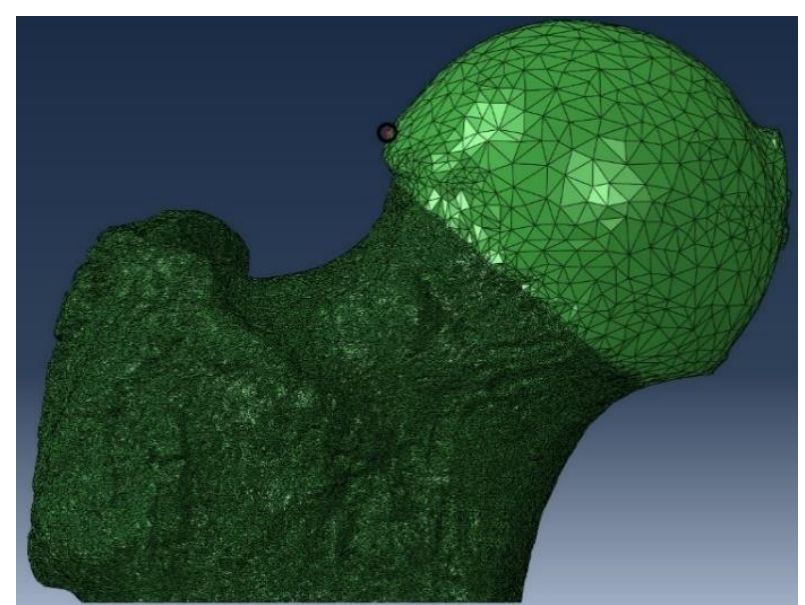

Figure 48: The red colored node inside a little circle chosen for convergence test in ABAQUS

Next, the same process was repeated with 10-noded elements each having more degrees of freedom than the 4-noded elements. The number of degrees of freedom (DOF) was then calculated for each model $\left(\mathrm{DOF}_{4}, \mathrm{DOF}_{10}\right)$ as three times the number of nodes because each node has three translational degrees of freedom:

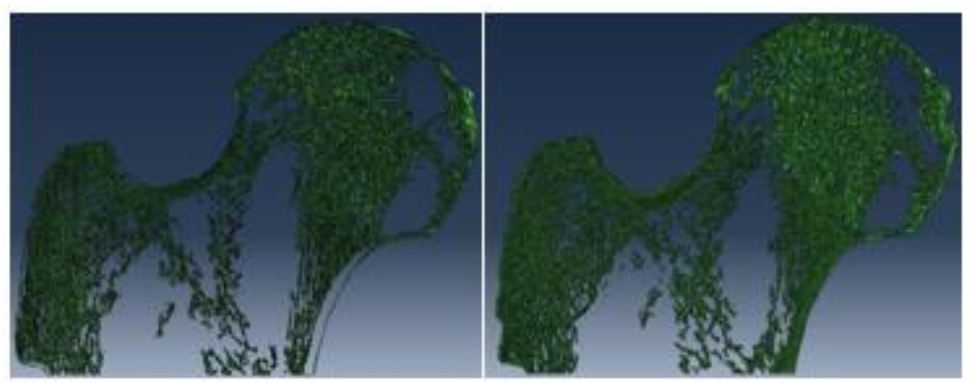

Figure 49: The 3D triangular surface in the left was converted to a 3D solid tetrahedral part in the right with 4-noded element mesh in ABAQUS

$$
D O F=\# \text { of nodes } * 3
$$

The two model types for the basic model (4 and 10 noded) were used for a convergence test to confirm the modeling approach was accurate before proceeding with the rest of the modeling validation and parametric study. Convergence was assessed by comparing the displacement of the same reference node in each model. Model convergence is a test of the mathematical accuracy of the modeling approach and should display increasing displacement with increasing DOF, but the change in 
displacement as DOF continues to increase should decrease as the calculated solution approaches (converges upon) a constant solution. Because of the limitations of the geometric size of the structures represented in these models (trabeculae) and the memory and computational limitations of the computer hardware and software, it was not possible to do more rigorous convergence testing in which the number of elements in the model was increased several-fold with smaller elements used at each iteration.

\subsubsection{Validation Methodology:}

To validate the basic computational model, the strain gauge strains at two load levels below the failure load level of the experiment were compared with the calculated strains from the basic computational model at the same location as the gauge on the superolateral cortex of the femoral neck. Our goal for being able to consider the FE model as validated was if the calculated strain was within $10 \%$ of the corresponding measured strain. When the basic FE model was run, the strain gauge location was determined by direct comparison to the experimental femur using the following steps:

The strain gauge center was located at $50 \mathrm{~mm}$ from the medial head, whereas the distance between the medial head and the trochanter was $90.8 \mathrm{~mm}$, as shown in the following figure.

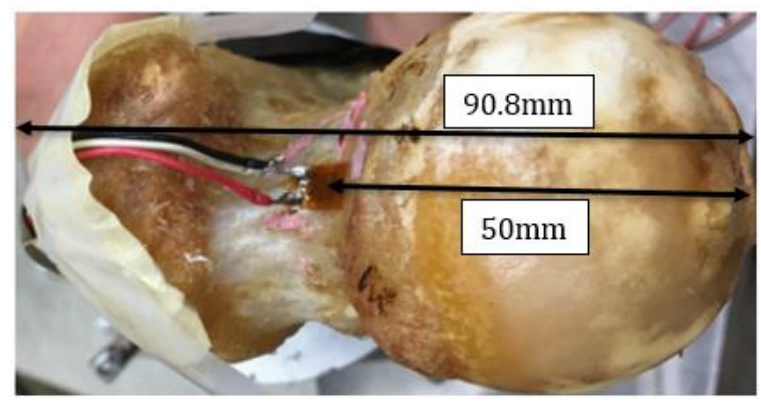

Figure 50: The strain gauge location measurements

The strain gauge grid size was $4 \mathrm{~mm} \times 10 \mathrm{~mm}$. The projection of this area at the orientation angle of the gauge in the frontal plane was used to identify the 
elements in the basic finite element model underlying the gauge grid pattern, see fig $(51)$.

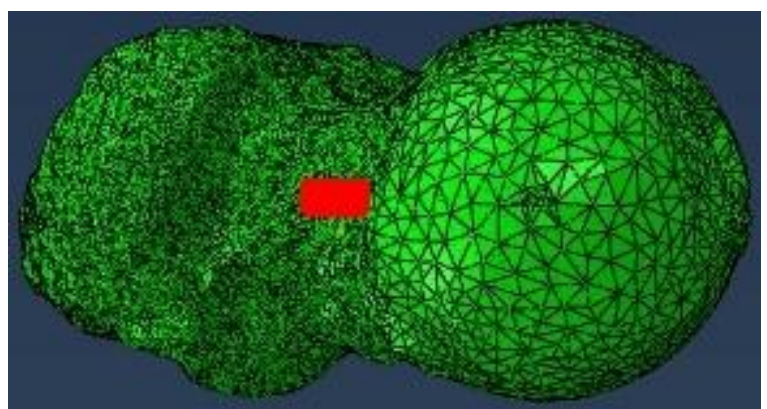

Figure 51: The strain gauge location determination in ABAQUS

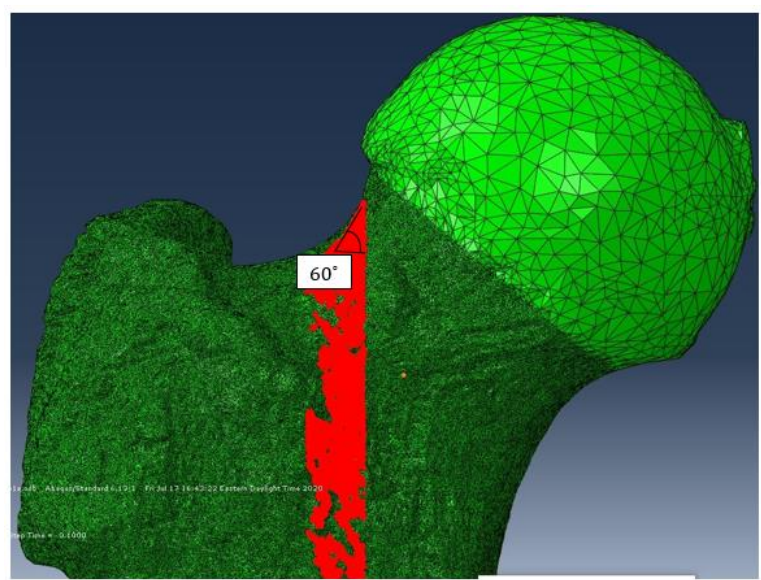

Figure 52: The strain gauge angle, of the basic FE model, measured virtually on the screen

The respective nodes located on the outer surface were selected for comparison of strain values between the FEA and the experiment.

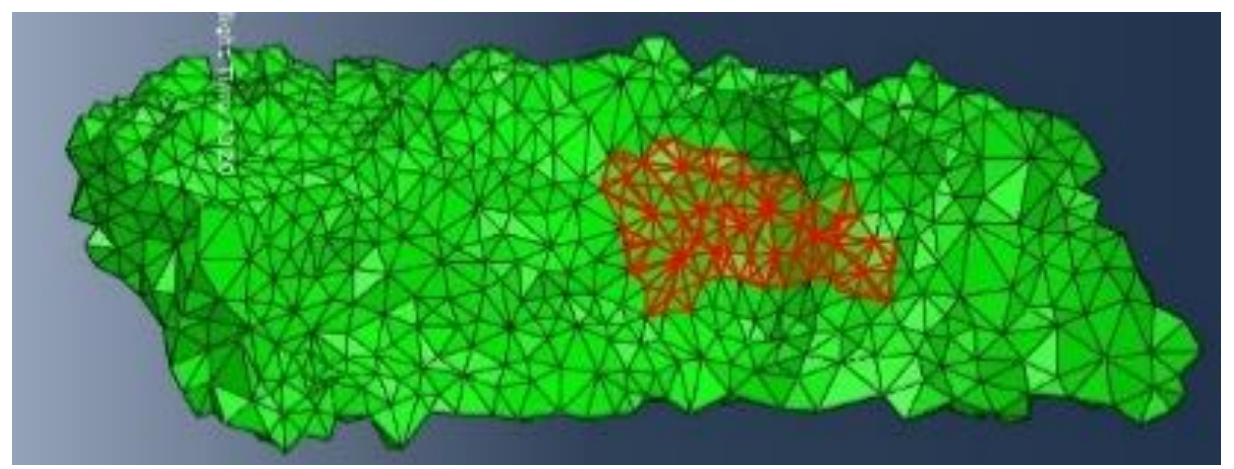

Figure 53: The green area represents the strain gauge location. The red highlighted elements were selected for calculations and analysis

The selection approach for the nodes was performed in ABAQUS using the tool called "Query>Probe values" so the nodes of interest were conveniently selected, and 
then reported for further calculations. The plane in which the strain gauge grid pattern lay was the 1-3 plane within the 3D space of the finite element model.

\subsubsection{Strain and Strain Energy Equations:}

The single strain value recorded by the strain gauge for each load level was oriented at an angle corresponding to the direction of the gauge grid within the 1-3 plane. The output file of the basic model in ABAQUS reports nine strain components at every node. The three components within the plane of the gauge, E11, E33, and E13 were used to calculate a resultant strain, $\varepsilon_{X}$, for each of the group of nodes identified as having been located on the bone surface located right under the strain gauge from the cadaver model. Excel was used to perform the strain transformation for each node using the following equation:

$$
\epsilon_{x^{\prime}}=\frac{\epsilon_{x}+\epsilon_{y}}{2}+\frac{\epsilon_{x}-\epsilon_{y}}{2} \times \cos 2 \theta+\frac{\gamma_{x y}}{2} \times \sin 2 \theta
$$

And Excel was also used to average these values of strain corresponding to the gauge

strain. Validation was performed by comparing the strains between FEA and experiment.

\subsubsection{Determination of Failure Criteria:}

Maximum compressive stress is expected to occur in the superolateral femoral neck while the thick inferomedial femoral neck is expected to experience tensile stress as illustrated in the following figure. 


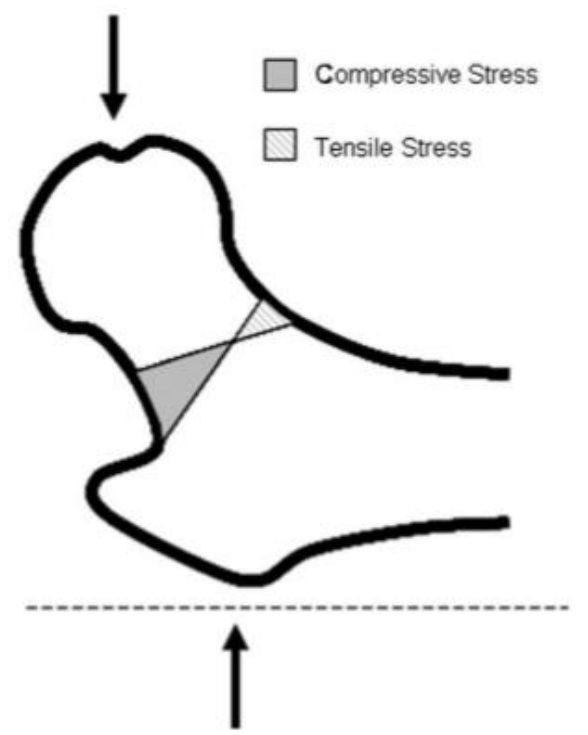

Figure 54: The expected resulted stresses distribution on the proximal femur due to sideways falling [68]

When the basic FE model has revealed valid results, the strain energy density (SED) was plotted for the femur at the second loading increments $(2000 \mathrm{~N})$. Next, the elements failed in excess of the effective strain value of $7000 \mu \varepsilon$ were displayed. However, this strain value cannot be directly reported from ABAQUS so it was converted to the corresponding SED value which can be calculated by the following equation.

$$
\epsilon_{e f f}=\sqrt{\frac{2 \times S E D}{E}}
$$

The E was assumed to be $10,000 \mathrm{MPa}$. Therefore,

$$
\begin{gathered}
S E D=\frac{\epsilon_{e f f^{2} \times E}}{2} \\
=(0.007)^{2 * 10000 / 2}=0.245 .
\end{gathered}
$$

From the SED plot, the elements which have SED value of 0.245 or greater were displayed in ABAQUS and reported into Excel after then. The list of reported elements included some elements with SED less than 0.245 so they were manually 
omitted from further calculations in Excel. Next, the total volume of the failed elements was calculated, and then was divided over the total volume of the bone tissue. Consequently, the percentage of bone tissue has failed at 0.245 or beyond when the whole bone just started to yield was calculated.

\subsection{FE Analysis for Altered Parameters:}

Similar to the basic model, eight FE models with varied morphological parameters underwent imaging processing, 2D to 3D meshed part conversion, and $\mathrm{FE}$ modeling including the same boundary and loading conditions. The SED for each of the eight FE models was plotted twice for two consecutive loading increments that revealed the closest lower and higher volume fraction than the calculated one for the basic model. The calculated fraction volume of the standard model was used as a standard in order to estimate every respective failure load for those eight FE models using linear interpolation method. In this way, the calculated standard percentage volume exceeding 0.245 in the basic FE model was determined and used in subsequent models to represent failure (fracture) load of the proximal femur using the linear interpolation method. The following figures show the conversion of the gray images in ImageJ to the 3D FE solid models in ABAQUS:
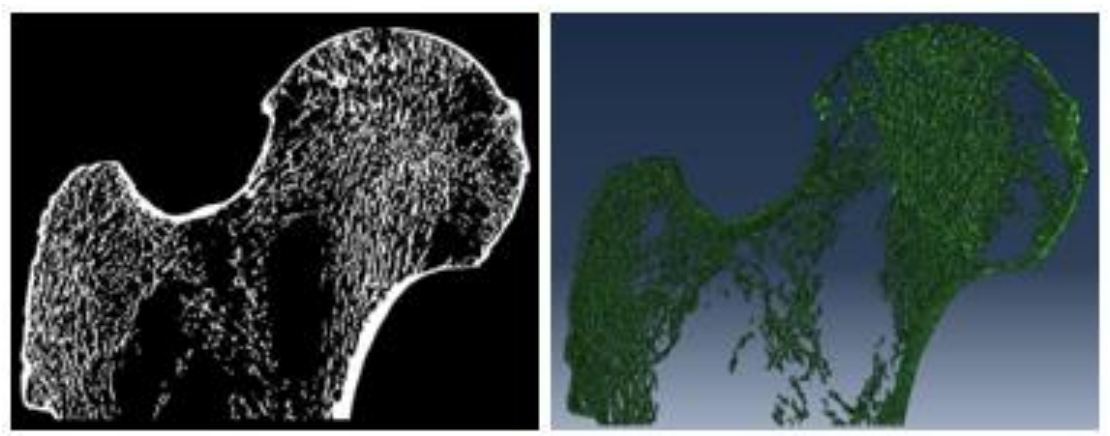

Figure 55: The left binary image in ImageJ, whereas the right image in ABAQUS. CD_TN: cortical dilated _ trabecular normal 

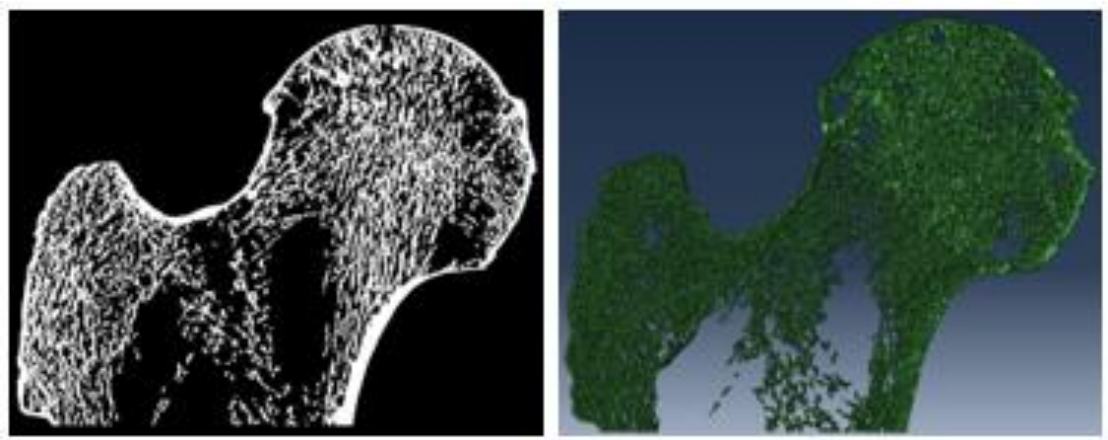

Figure 56: The left binary image in ImageJ, whereas the right image in ABAQUS. CD_TD: cortical dilated _ trabecular dilated
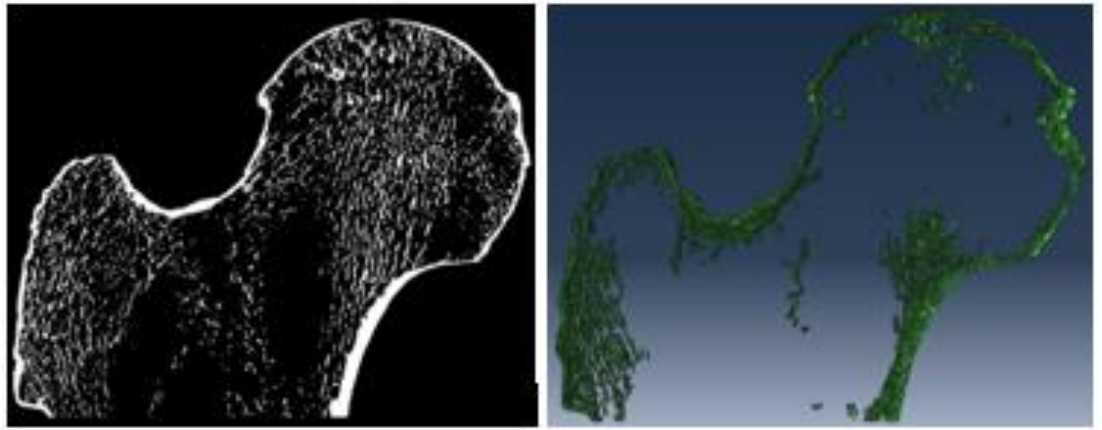

Figure 57: The left binary image in ImageJ, whereas the right image in ABAQUS. CD_TE: cortical dilated _ trabecular eroded
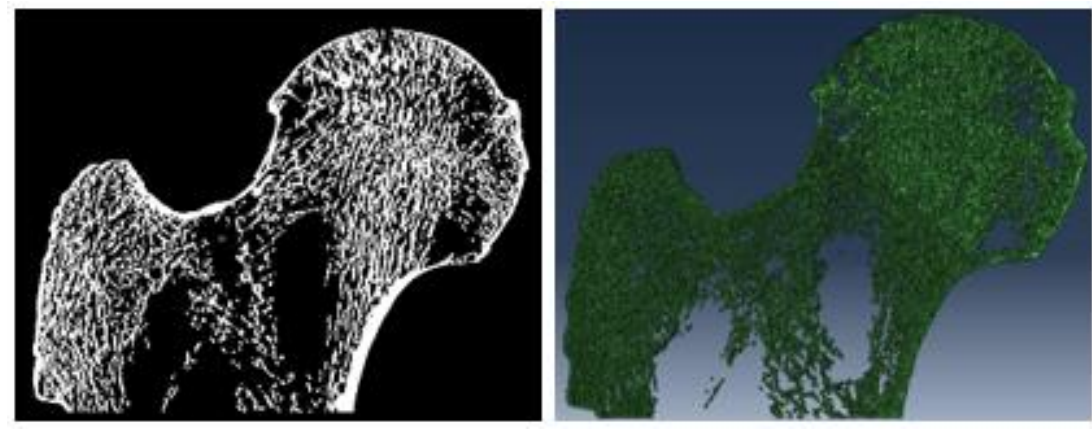

Figure 58: The left binary image in ImageJ, whereas the right image in ABAQUS. CN_TD: cortical normal _ trabecular dilated
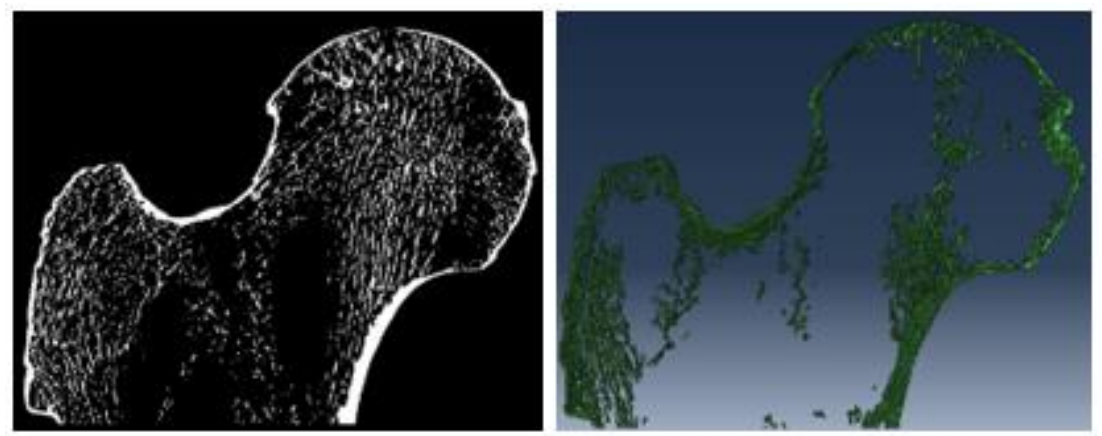

Figure 59: The left binary image in ImageJ, whereas the right image in ABAQUS. CN_TE: cortical normal _ trabecular eroded 

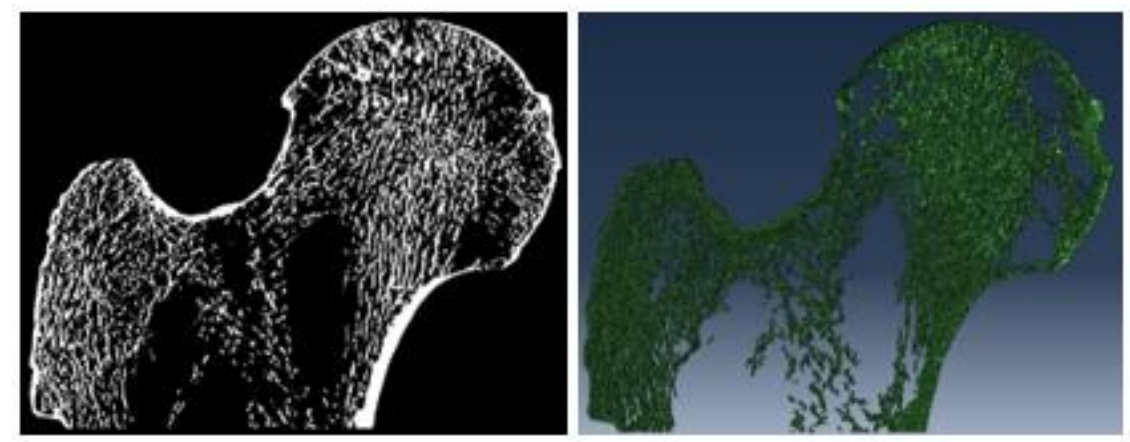

Figure 60: The left binary image in ImageJ, whereas the right image in ABAQUS. CE_TN: cortical eroded _ trabecular normal
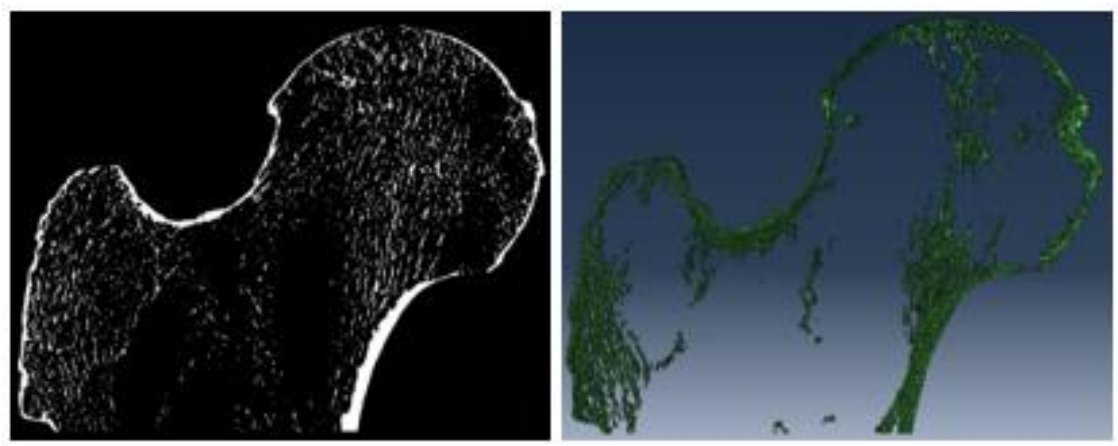

Figure 61: The left binary image in ImageJ, whereas the right image in ABAQUS. CE_TE: cortical eroded _ trabecular eroded
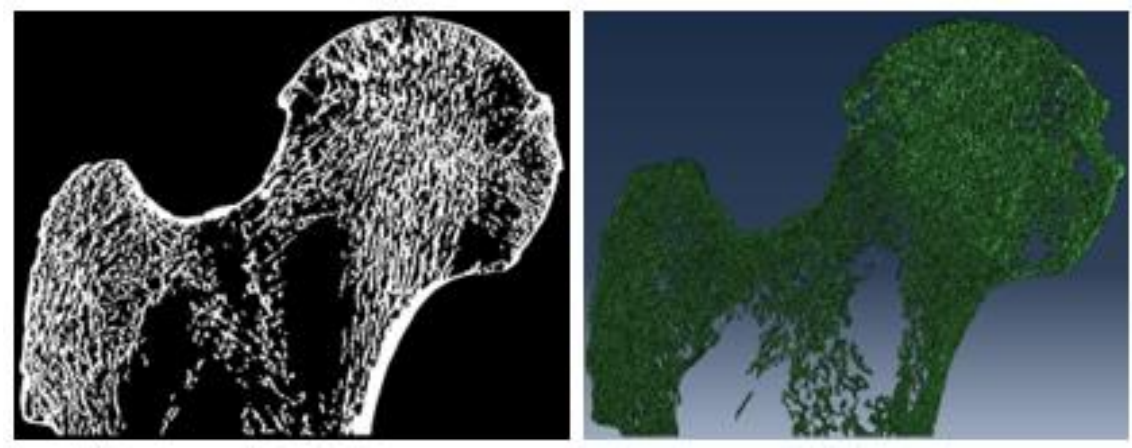

Figure 62: The left binary image in ImageJ, whereas the right image in ABAQUS. CE_TD: cortical eroded _ trabecular dilated 


\section{CHAPTER 4: RESULTS}

This chapter presents the experimental data and computational results of nine FE models including the basic model. The fall-to-the-side fracture experiment results are described in the first section, 4.1, and the strain gauge measured surface strains, the failure load when the femoral neck fracture occurred, and the fracture pattern of the femoral neck are presented. In section 4.2, the basic finite element model computational results are presented. The model convergence, validation of the basic model by direct comparison of calculated strain to the experimental strain measurements, and the determination of the elastic strain-based failure criteria are all described. The final section, 4.3, shows the parametric analysis results with the increases or decreases of the failure load values for each of the additional eight systematically dilated and/or eroded FE models.

\subsection{Experimental Results:}

The experiment in which the cadaver femur was loaded in a simulated fall-tothe-side configuration exhibited a force-time and three strain-time curves that gradually increased in slope until a linear increase was followed by a first peak (local minima) followed by a final peak (ultimate load) representing complete fracture of the femoral neck (Table 1, Figure 63, Figure 64). The bone in the superolateral cortex started to fail at the first peak load of $-2140 \mathrm{~N}$ (compression). Negative strain gauge output values indicate that the anatomical site at which a strain gauge was located was subjected to compression loading, whereas positive strain values represent tension. 
Table 1: Measured yield force of $-\mathbf{2 1 4 0 N}$

\begin{tabular}{|c|c|c|c|c|}
\hline Time (sec) & Force $(\mathrm{N})$ & strain 1 & Strain 3 & Strain 4 \\
\hline 0.280625 & -1949.400000 & 1800.286000 & -1285.880000 & 2508.327000 \\
\hline 0.281601 & -1996.340000 & 1841.969000 & -1312.410000 & 2583.429000 \\
\hline 0.282577 & -2046.720000 & 1890.886000 & -1316.200000 & 2649.918000 \\
\hline 0.283553 & -2096.670000 & 1936.358000 & -1344.440000 & 2724.675000 \\
\hline 0.284529 & -2140.160000 & 1972.873000 & -1373.380000 & 2792.886000 \\
\hline 0.285505 & -2063.080000 & 1868.839000 & -1296.910000 & 2668.865000 \\
\hline
\end{tabular}

Sideways fracture test for a human proximal femur

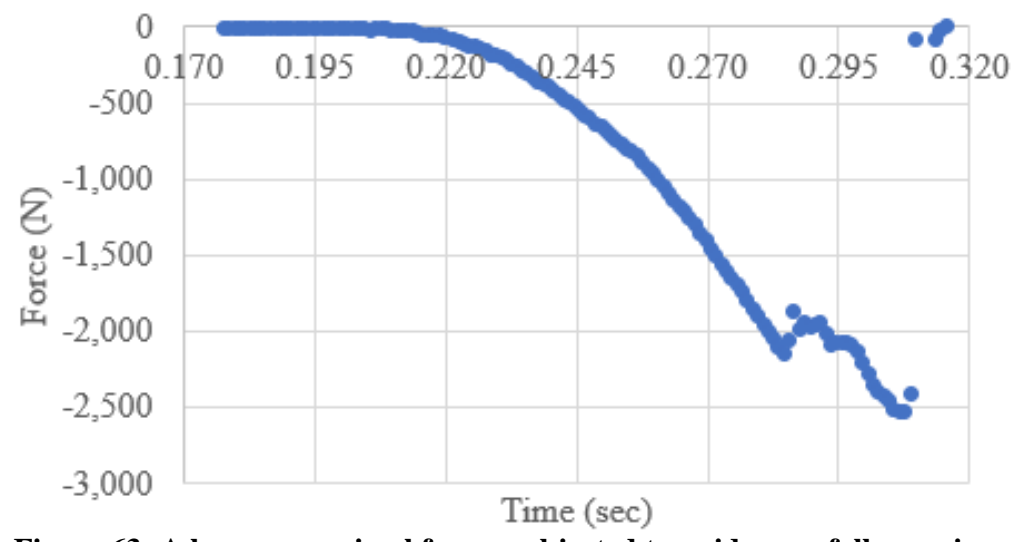

Figure 63: A human proximal femur subjected to a sideways fall experimental test. The curve illustrates the femur behavior represented by force(N) vs. time(sec).

Sideways fracture test for a human proximal femur

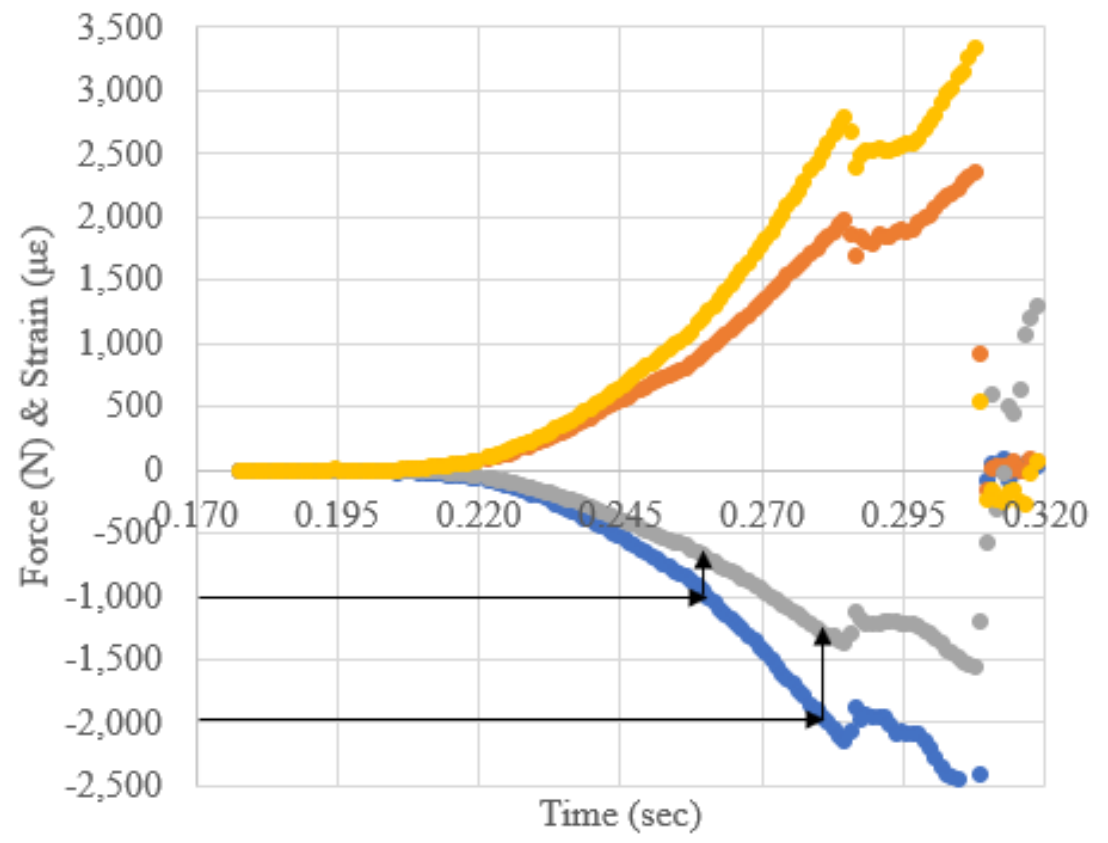

- Force $(\mathrm{N})$ - strain 1 - Strain 3 - Strain 4

Figure 64: The chart illustrates the experimental data of time vs force and 3 strains. The arrows point to strain values reported from strain gauge \#3 at both loading increments of $1000 \mathrm{~N}$ and $2000 \mathrm{~N}$ 


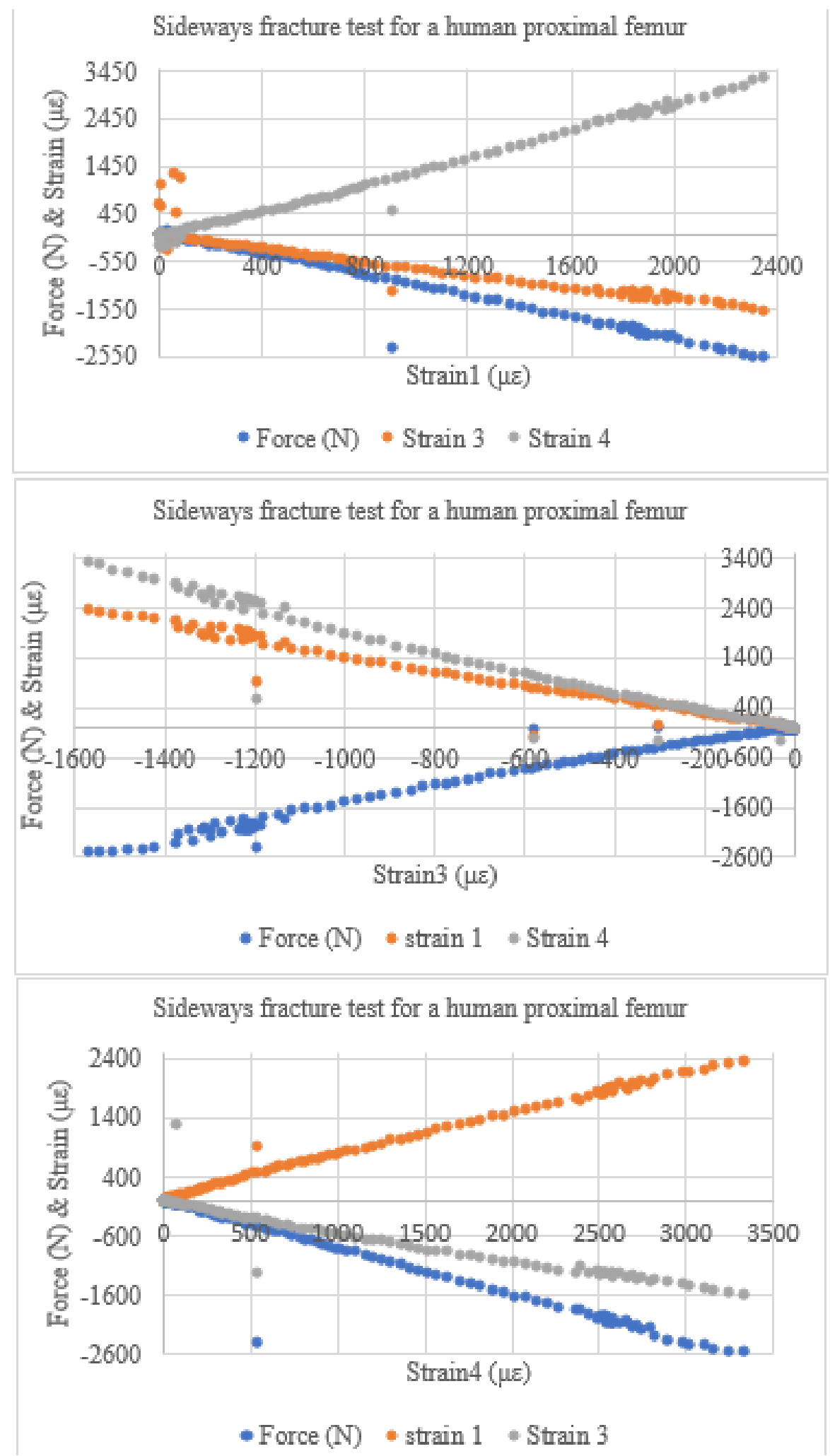

Figure 65: Experimental results revealed linear relationships between values of each strain gauge and corresponding values of force and other strain gauges 
The following figure shows femur when it was completely broken. The blue arrow points to the probable location for the failure initiation (Figure 66). The red arrows indicate the location of Gauge 3.

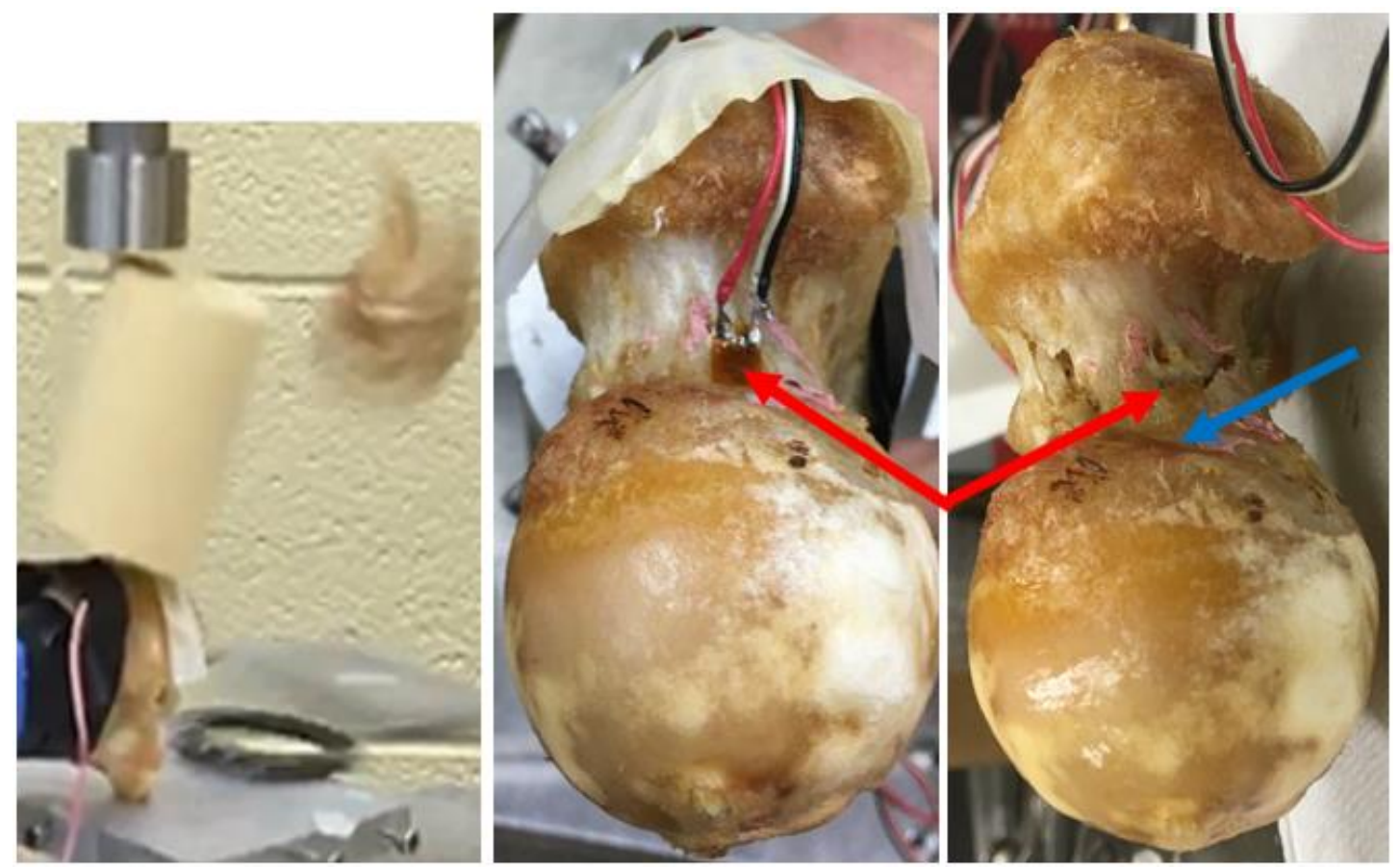

Figure 66: The Femur when it was completely broken. The red arrows indicate the location of gauge 3 before and after breakage, whereas the blue arrow points to the probable failure initiation

\subsection{FE Basic Model Execution Results:}

\subsubsection{Convergence Test Results:}

Based on the convergence test method explained in the previous chapter, the basic FE model with 10-noded tetrahedral element mesh revealed a higher displacement than the 4-noded element mesh at $1000 \mathrm{~N}$ and $2000 \mathrm{~N}$ as follows: 


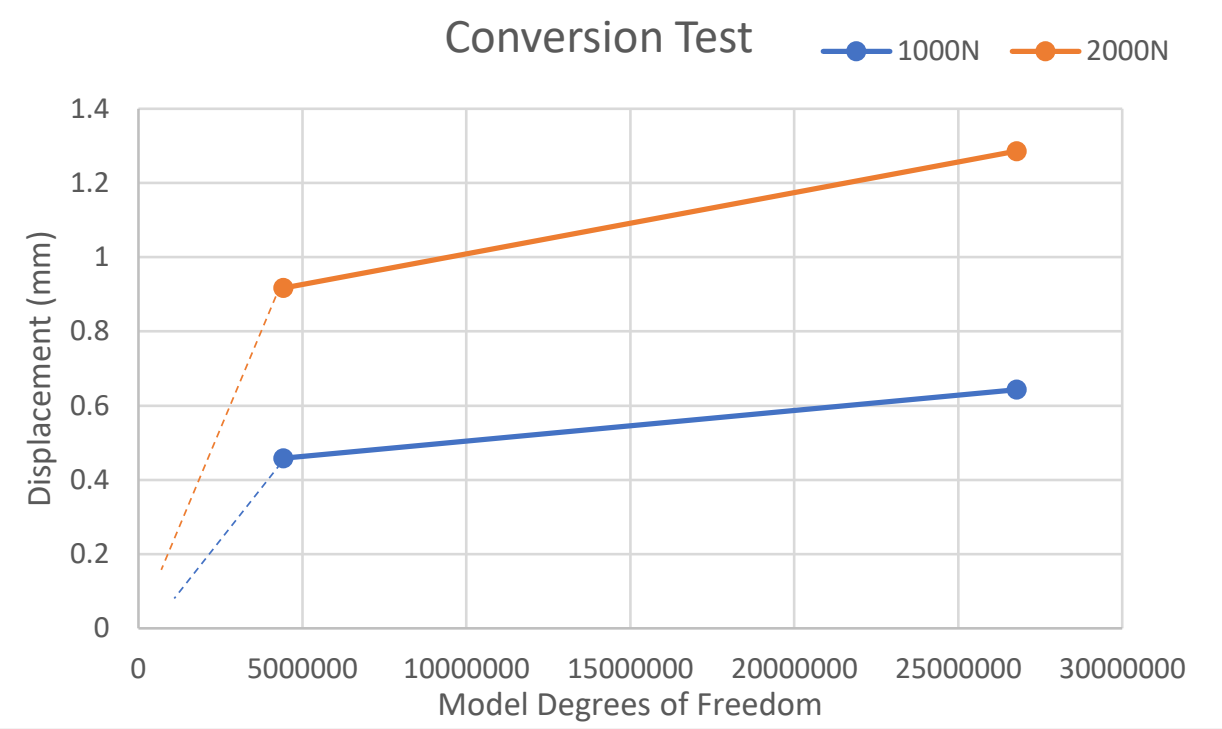

Figure 67: The chart represents displacement vs. model degrees of freedom for both models, 4-noded element mesh $(4,413,027$ DOF) and 10-noded element mesh $(26,787,105$ DOF $)$ at the first loading increment $(1000 \mathrm{~N})$ and the second loading increment $(2000 \mathrm{~N})$

The 4-noded element mesh with 1,471,009 nodes and 4,413,027 DOF displaced $0.46 \mathrm{~mm}$ at $1000 \mathrm{~N}$ and $0.92 \mathrm{~mm}$ at $2000 \mathrm{~N}$. However, the 10-noded element mesh with $8,929,035$ nodes and 26,787,105 DOF displaced $0.64 \mathrm{~mm}$ at $1000 \mathrm{~N}$ and $1.29 \mathrm{~mm}$ at 2000 N. Although it was not possible to execute models with either fewer or more degrees of freedom, it appears that this model geometry would have converged to an exact solution as degrees of freedom continued to increase.

\subsubsection{Validation Testing Results:}

From the contour plot of the principal strains on the basic model at a load level of $2000 \mathrm{~N}$, it can be seen that there is a wide variation in strain values on the surface of the bone that was covered by the stain gauge in the corresponding cadaver experiment (Figure 67). Thus, the strain components from all of the elements "covered" by the gauge grid (Figure 68) were exported from the ABAQUS output file and used with equation (3.2) to calculate the resultant strain parallel to the surface orientation of the gauge (Table 2). 


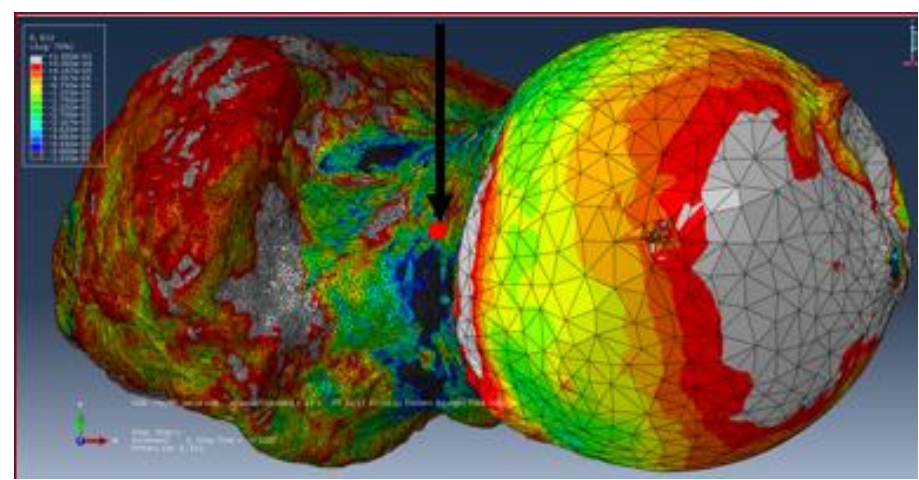

Figure 68: The resulted strain contour where the maximum compressive strain regions shown with a black color, whereas the maximum tension strain shown with a gray color. The black arrow points to the location of the strain gauge \#3

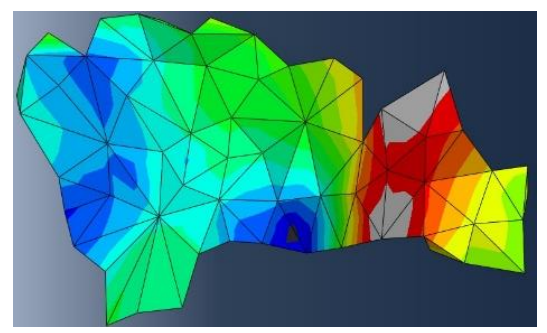

Figure 69: The group of elements selected from the red highlighted region in the previous figure, which represents the location of the strain gauge \#3

The ABAQUS output files containing the strain components for all of the elements representing the corresponding locations on the model for both strain gauges \#3 and \#4 were transferred to Excel in order to calculate the mean values of the strains E11, E33, E13, and then perform the transformation calculation of the resultant strain $\varepsilon x$ ' for comparison to the measured strain gauge strains from the experiment. The surface angle of each strain gauge location on the model was manually measured and also used along for the calculation of $\varepsilon_{X}$ ' (Tables $2 \& 3$ ): 
Table 2: Values reported from ABAQUS into Excel for strain gauge \#3. The variables E11, E33, E13, and Ex' represent normal and shear strains and resultant strain as the strain gauge angle was included in calculations.

\begin{tabular}{|c|c|c|c|c|}
\hline NodeID & E11 & E33 & E13 & $\varepsilon x^{\prime}$ \\
\hline 1009033 & $1.29 \mathrm{E}-03$ & $2.87 \mathrm{E}-04$ & $-2.17 \mathrm{E}-03$ & -0.0004019 \\
\hline 5088186 & $1.03 \mathrm{E}-03$ & $1.52 \mathrm{E}-04$ & $-2.60 \mathrm{E}-03$ & -0.0007543 \\
\hline 5071888 & $1.05 \mathrm{E}-03$ & $-2.73 \mathrm{E}-04$ & $-2.55 \mathrm{E}-03$ & -0.0010464 \\
\hline 5025721 & $1.13 \mathrm{E}-03$ & $-7.11 \mathrm{E}-06$ & $-2.44 \mathrm{E}-03$ & -0.0007794 \\
\hline 5025723 & $1.41 \mathrm{E}-03$ & $5.48 \mathrm{E}-04$ & $-2.19 \mathrm{E}-03$ & -0.0001848 \\
\hline 5182258 & $1.67 \mathrm{E}-03$ & $9.54 \mathrm{E}-04$ & $-1.00 \mathrm{E}-03$ & 0.00069999 \\
\hline 5224778 & $1.37 \mathrm{E}-03$ & 8.79E-04 & $-1.42 \mathrm{E}-03$ & 0.00038687 \\
\hline 1003620 & $1.97 \mathrm{E}-03$ & $1.12 \mathrm{E}-03$ & $3.60 \mathrm{E}-04$ & 0.00148838 \\
\hline 4714973 & $1.78 \mathrm{E}-03$ & $1.23 \mathrm{E}-03$ & $2.75 \mathrm{E}-04$ & 0.00148658 \\
\hline 5119695 & $1.61 \mathrm{E}-03$ & $9.88 \mathrm{E}-04$ & $-9.50 \mathrm{E}-04$ & 0.00073214 \\
\hline 4714990 & $1.28 \mathrm{E}-03$ & $1.08 \mathrm{E}-03$ & $-3.83 \mathrm{E}-05$ & 0.00111342 \\
\hline 4714989 & $1.11 \mathrm{E}-03$ & $1.22 \mathrm{E}-03$ & $4.85 \mathrm{E}-04$ & 0.00140251 \\
\hline 2240227 & $1.97 \mathrm{E}-03$ & $4.18 \mathrm{E}-04$ & $5.87 \mathrm{E}-04$ & 0.00106018 \\
\hline 2240223 & $2.30 \mathrm{E}-03$ & $3.86 \mathrm{E}-04$ & $1.55 \mathrm{E}-03$ & 0.00153567 \\
\hline 998050 & $1.89 \mathrm{E}-03$ & $-3.86 \mathrm{E}-04$ & 8.47E-04 & 0.00054976 \\
\hline 2240225 & $2.23 \mathrm{E}-03$ & $-5.61 \mathrm{E}-04$ & $1.28 \mathrm{E}-03$ & 0.00069101 \\
\hline 5198162 & $9.04 \mathrm{E}-04$ & $6.25 \mathrm{E}-04$ & $7.53 \mathrm{E}-04$ & 0.00102081 \\
\hline 5039956 & $1.56 \mathrm{E}-03$ & $-2.12 \mathrm{E}-04$ & $1.07 \mathrm{E}-03$ & 0.00069432 \\
\hline 5039952 & $1.91 \mathrm{E}-03$ & $-1.43 \mathrm{E}-03$ & $3.76 \mathrm{E}-04$ & -0.0004322 \\
\hline 5242908 & $2.29 \mathrm{E}-03$ & $-1.87 \mathrm{E}-03$ & $2.63 \mathrm{E}-04$ & -0.0007161 \\
\hline 994422 & $2.79 \mathrm{E}-03$ & $-3.51 \mathrm{E}-03$ & $-8.59 \mathrm{E}-04$ & -0.002307 \\
\hline 4298276 & $2.93 \mathrm{E}-03$ & $-2.29 \mathrm{E}-03$ & $9.97 \mathrm{E}-04$ & 5533 \\
\hline 5298503 & $2.28 \mathrm{E}-03$ & $-3.07 \mathrm{E}-03$ & $-1.07 \mathrm{E}-03$ & -0.00 \\
\hline 2233506 & $2.84 \mathrm{E}-03$ & $-4.67 \mathrm{E}-03$ & $-4.26 \mathrm{E}-03$ & -0.0046371 \\
\hline 2233508 & $3.71 \mathrm{E}-03$ & $-4.44 \mathrm{E}-03$ & $-2.73 \mathrm{E}-03$ & -0.0035846 \\
\hline 994320 & $2.03 \mathrm{E}-03$ & $-2.36 \mathrm{E}-03$ & $-1.30 \mathrm{E}-03$ & -0.0018254 \\
\hline 5039954 & $2.03 \mathrm{E}-03$ & $-1.53 \mathrm{E}-03$ & $-4.62 \mathrm{E}-06$ & -0.000642 \\
\hline 5224837 & $2.44 \mathrm{E}-03$ & $-2.10 \mathrm{E}-03$ & $-1.44 \mathrm{E}-03$ & -0.0015885 \\
\hline 5182130 & $1.75 \mathrm{E}-03$ & $-2.15 \mathrm{E}-03$ & $-3.12 \mathrm{E}-03$ & -0.002526 \\
\hline 4298268 & $2.09 \mathrm{E}-03$ & $-3.14 \mathrm{E}-03$ & $-4.13 \mathrm{E}-03$ & -0.0036208 \\
\hline 990391 & $1.97 \mathrm{E}-03$ & $-3.39 \mathrm{E}-03$ & $-7.11 \mathrm{E}-03$ & -0.0051287 \\
\hline 2233504 & $2.72 \mathrm{E}-03$ & $-4.10 \mathrm{E}-03$ & $-6.39 \mathrm{E}-03$ & -0.005162 \\
\hline 3358390 & $1.34 \mathrm{E}-03$ & $-2.33 \mathrm{E}-03$ & $-5.68 \mathrm{E}-03$ & -0.003872 \\
\hline 3358386 & $1.03 \mathrm{E}-03$ & $-2.40 \mathrm{E}-03$ & $-8.08 \mathrm{E}-03$ & -0.0050412 \\
\hline 5364120 & $1.55 \mathrm{E}-03$ & $-3.10 \mathrm{E}-03$ & $-8.81 \mathrm{E}-03$ & -0.0057523 \\
\hline 987432 & $1.22 \mathrm{E}-03$ & $-1.70 \mathrm{E}-03$ & $-4.40 \mathrm{E}-03$ & -0.0028753 \\
\hline 5331418 & $1.99 \mathrm{E}-03$ & $-1.26 \mathrm{E}-03$ & $-2.81 \mathrm{E}-03$ & -0.0016643 \\
\hline 5331415 & $2.03 \mathrm{E}-03$ & $-1.13 \mathrm{E}-03$ & $-3.32 \mathrm{E}-03$ & -0.0017776 \\
\hline 2730303 & $1.34 \mathrm{E}-03$ & $-1.12 \mathrm{E}-03$ & $-4.23 \mathrm{E}-03$ & -0.0023366 \\
\hline 2730286 & $6.43 \mathrm{E}-04$ & $-1.28 \mathrm{E}-03$ & $-4.62 \mathrm{E}-03$ & -0.0027998 \\
\hline 2730285 & $6.93 \mathrm{E}-04$ & $-1.52 \mathrm{E}-03$ & $-5.05 \mathrm{E}-03$ & -0.0031535 \\
\hline 5445617 & $9.43 \mathrm{E}-04$ & $-1.78 \mathrm{E}-03$ & $-5.25 \mathrm{E}-03$ & -0.0033726 \\
\hline 3358388 & 7.79E-04 & $-1.72 \mathrm{E}-03$ & $-5.96 \mathrm{E}-03$ & -0.003676 \\
\hline mean & $1.74 \mathrm{E}-03$ & $-1.18 \mathrm{E}-03$ & $-2.26 \mathrm{E}-03$ & $-1.43 \mathrm{E}-03$ \\
\hline $\mathrm{SD}$ & $6.73 \mathrm{E}-04$ & $1.67 \mathrm{E}-03$ & $2.73 \mathrm{E}-03$ & $2.12 \mathrm{E}-03$ \\
\hline theta= & & $\varepsilon x^{\prime}$ & & \\
\hline 1.047198 & & $-1.4313 \mathrm{E}-03$ & & \\
\hline
\end{tabular}


Table 3: Values reported from ABAQUS into Excel for strain gauge \#4. The variables E11, E33, E13, and Ex' represent normal and shear strains and resultant strain as the strain gauge angle was included in calculations

\begin{tabular}{|c|c|c|c|c|}
\hline Node & E11 & E33 & E13 & $\varepsilon x^{\prime}=$ \\
\hline 47529 & $-3.55 E-03$ & $7.63 \mathrm{E}-03$ & $4.60 \mathrm{E}-03$ & $7.98 \mathrm{E}-03$ \\
\hline 39068 & $-3.45 E-03$ & 7.30E-03 & $3.56 \mathrm{E}-03$ & $7.55 \mathrm{E}-03$ \\
\hline 33157 & $-3.44 \mathrm{E}-03$ & $6.63 \mathrm{E}-03$ & $2.96 \mathrm{E}-03$ & $6.83 \mathrm{E}-03$ \\
\hline 23787 & $-3.27 \mathrm{E}-03$ & 7.93E-03 & $4.21 \mathrm{E}-03$ & $8.25 \mathrm{E}-03$ \\
\hline 24068 & $-3.51 E-03$ & $7.84 \mathrm{E}-03$ & $4.04 \mathrm{E}-03$ & $8.14 \mathrm{E}-03$ \\
\hline 18242 & $-3.39 E-03$ & $7.65 \mathrm{E}-03$ & $2.16 \mathrm{E}-03$ & 7.76E-03 \\
\hline 10208 & $-2.07 \mathrm{E}-03$ & $6.77 \mathrm{E}-03$ & $4.41 \mathrm{E}-03$ & 7.14E-03 \\
\hline 679 & $-4.00 \mathrm{E}-03$ & $2.30 \mathrm{E}-02$ & $3.38 \mathrm{E}-03$ & $2.31 \mathrm{E}-02$ \\
\hline 1524 & $-1.13 \mathrm{E}-02$ & 1.47E-02 & 4.57E-04 & $1.45 \mathrm{E}-02$ \\
\hline 320 & $-1.01 E-02$ & $1.23 \mathrm{E}-03$ & $2.28 \mathrm{E}-03$ & 1.35E-03 \\
\hline 321 & $-6.13 \mathrm{E}-03$ & 4.57E-03 & $-2.16 \mathrm{E}-03$ & $4.23 \mathrm{E}-03$ \\
\hline 4319 & $-9.29 E-03$ & $1.67 \mathrm{E}-02$ & $5.09 \mathrm{E}-03$ & 1.69E-02 \\
\hline 7716 & $-6.01 \mathrm{E}-03$ & $9.41 \mathrm{E}-03$ & $6.13 \mathrm{E}-03$ & $9.88 \mathrm{E}-03$ \\
\hline 8749 & $-3.74 \mathrm{E}-03$ & $5.80 \mathrm{E}-03$ & 4.69E-03 & 6.18E-03 \\
\hline 14761 & $-5.30 \mathrm{E}-03$ & $1.01 \mathrm{E}-02$ & $2.68 \mathrm{E}-03$ & $1.02 \mathrm{E}-02$ \\
\hline 20002 & $-4.97 \mathrm{E}-03$ & $1.05 \mathrm{E}-02$ & $3.25 \mathrm{E}-03$ & $1.06 \mathrm{E}-02$ \\
\hline 20461 & $-4.96 \mathrm{E}-03$ & $9.98 \mathrm{E}-03$ & 3.49E-03 & $1.02 \mathrm{E}-02$ \\
\hline 25095 & $-4.56 \mathrm{E}-03$ & $9.88 \mathrm{E}-03$ & $4.71 \mathrm{E}-03$ & $1.02 \mathrm{E}-02$ \\
\hline 31216 & $-3.91 \mathrm{E}-03$ & 7.90E-03 & 4.47E-03 & 8.24E-03 \\
\hline 37259 & $-3.77 \mathrm{E}-03$ & 8.03E-03 & $4.46 \mathrm{E}-03$ & 8.36E-03 \\
\hline 45275 & $-3.57 \mathrm{E}-03$ & 7.31E-03 & $4.38 \mathrm{E}-03$ & $7.65 \mathrm{E}-03$ \\
\hline 46306 & $-3.53 \mathrm{E}-03$ & $7.21 \mathrm{E}-03$ & $4.48 \mathrm{E}-03$ & $7.56 \mathrm{E}-03$ \\
\hline 47756 & $-3.69 \mathrm{E}-03$ & $7.67 \mathrm{E}-03$ & 4.67E-03 & 8.03E-03 \\
\hline 37059 & $-3.61 E-03$ & $7.14 \mathrm{E}-03$ & $3.62 \mathrm{E}-03$ & $7.40 \mathrm{E}-03$ \\
\hline 37130 & $-3.71 \mathrm{E}-03$ & 7.53E-03 & 4.09E-03 & 7.83E-03 \\
\hline 28977 & $-3.33 \mathrm{E}-03$ & $6.28 \mathrm{E}-03$ & 3.98E-03 & 6.59E-03 \\
\hline 25669 & $-3.87 \mathrm{E}-03$ & 7.33E-03 & 3.80E-03 & 7.60E-03 \\
\hline 24510 & $-4.47 \mathrm{E}-03$ & 8.87E-03 & 4.17E-03 & $9.16 \mathrm{E}-03$ \\
\hline 21355 & $-3.84 \mathrm{E}-03$ & 7.71E-03 & 3.43E-03 & $7.94 \mathrm{E}-03$ \\
\hline 16530 & $-3.26 \mathrm{E}-03$ & 6.62E-03 & $2.20 \mathrm{E}-03$ & 6.74E-03 \\
\hline 14385 & $-2.94 \mathrm{E}-03$ & $4.40 \mathrm{E}-03$ & $2.54 \mathrm{E}-03$ & $4.58 \mathrm{E}-03$ \\
\hline 17539 & $-4.24 \mathrm{E}-03$ & $8.20 \mathrm{E}-03$ & $1.82 \mathrm{E}-03$ & 8.25E-03 \\
\hline 11861 & $-2.33 \mathrm{E}-03$ & $2.57 \mathrm{E}-03$ & $2.24 \mathrm{E}-03$ & $2.75 \mathrm{E}-03$ \\
\hline 12287 & $-3.17 \mathrm{E}-03$ & \begin{tabular}{|l|}
$6.88 \mathrm{E}-03$ \\
\end{tabular} & $3.29 \mathrm{E}-03$ & $7.11 \mathrm{E}-03$ \\
\hline 7027 & $-2.04 \mathrm{E}-03$ & 8.97E-03 & $-1.12 \mathrm{E}-03$ & 8.74E-03 \\
\hline 9027 & $-4.07 \mathrm{E}-03$ & $5.21 \mathrm{E}-03$ & 2.71E-03 & 5.39E-03 \\
\hline 10254 & $-4.47 \mathrm{E}-03$ & 9.36E-03 & $5.11 \mathrm{E}-03$ & 9.74E-03 \\
\hline 5454 & $-5.70 \mathrm{E}-03$ & 8.70E-03 & $1.54 \mathrm{E}-02$ & 1.01E-02 \\
\hline mean & $-4.38 \mathrm{E}-03$ & $8.20 \mathrm{E}-03$ & 3.67E-03 & 8.44E-03 \\
\hline stdev & 1.97E-03 & $3.66 \mathrm{E}-03$ & $2.54 \mathrm{E}-03$ & $3.65 \mathrm{E}-03$ \\
\hline theta $=$ & 1.46608 & & $\varepsilon X^{\prime}=$ & $8.44 \mathrm{E}-03$ \\
\hline
\end{tabular}


To reach the same accurate level of experimental results, the femur model only has to be meshed into higher divisions corresponding with the scanning resolution of 60-microns for validation test and then for predicting the failure load. Because of the software limitations for size of models, the $10 \%$ was specified as acceptable limit for calculated results.

The strain gauge $\varepsilon 4$ was excluded from remaining calculations and analyses because the calculated effective strain values in both loading increments of $1000 \mathrm{~N}$ and $2000 \mathrm{~N}$ differed from the measured ones by more than $10 \%$, which is the maximum limit for error percentage as shown in tables 4 and 5 .

Table 4: Excel calculations at $1000 \mathrm{~N}$ for the calculated and measured strains for the strain gauges 3 and 4

\begin{tabular}{|c|c|c|c|}
\hline $1000 \mathrm{~N}$ & EXP. $\mu \varepsilon$ & $\mathrm{FE} \mu \varepsilon$ & error $\%$ \\
\hline$\varepsilon 3$ & -701 & -715 & $2.1 \%$ \\
\hline$\varepsilon 4$ & 1253 & 4272 & $241.0 \%$ \\
\hline
\end{tabular}

Table 5: Excel calculations at $2000 \mathrm{~N}$ for the calculated and measured strains for the strain gauges 3 and 4

\begin{tabular}{|c|c|c|c|}
\hline $2000 \mathrm{~N}$ & EXP. $\mu \varepsilon$ & $\mathrm{FE} \mu \varepsilon$ & error $\%$ \\
\hline$\varepsilon 3$ & -1313 & -1431 & $9.0 \%$ \\
\hline$\varepsilon 4$ & 2583 & 8443 & $226.9 \%$ \\
\hline
\end{tabular}

Therefore, the computational analysis and calculations were completed with using the strain gauge $\varepsilon 3$ results only. At the matching locations and orientations, the FE model calculated strain was within $10 \%$ of the measured strain at both $1000 \mathrm{~N}$ and $2000 \mathrm{~N}$ load levels to satisfy the validation requirement.

\subsubsection{FE Failure Criteria:}

The failure criteria were developed by calculating the volume fraction of the bone tissue in the basic model with an effective strain above $7000 \mu \varepsilon$ at the 
experimental failure load (actually $2000 \mathrm{~N}$, which was the load level at which a full model output file was generated). To perform this calculation using the ABAQUS output file, the effective strain of $7000 \mu \varepsilon$ was converted to its corresponding strain energy density (SED) value of 0.245 using Equation (3.3). The contour plot of SED for the basic model at $2000 \mathrm{~N}$ loading shows where the tissue "yielding" is taking place (Figure 70). The damage areas are shown as gray regions.

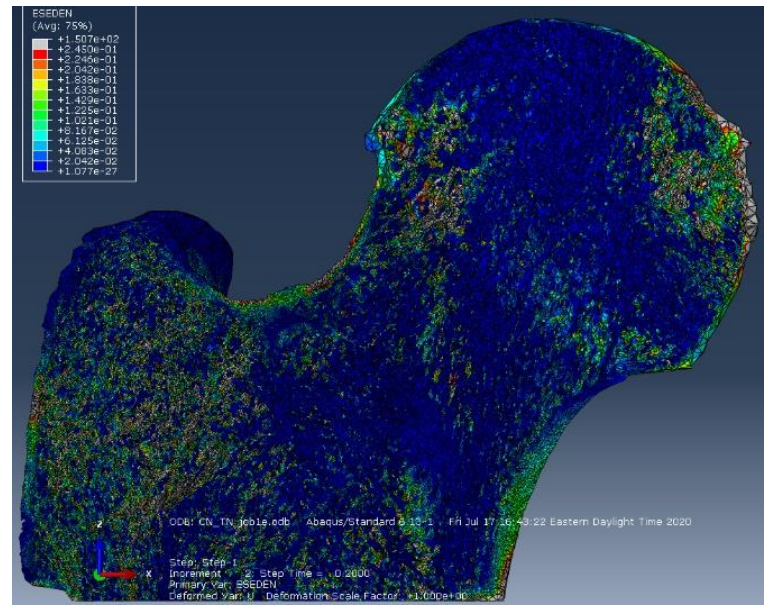

Figure 70: Frontal section of SED plot for the basic FE model at 2000N

An alternative plot was created using only elements containing nodes with SED values of 0.245 or higher (Figure 71). A complete list of these elements was taken from the ABAQUS output file and transferred to Excel where the data were sorted to determine the volume of elements with an SED over 0.245 and the corresponding volume fraction was calculated by dividing by the total model element volume. The failure criterion of percent model volume with effective strain of $7000 \mu \varepsilon$ or greater was thus determined to be $4.2 \%$ (Table 6). 


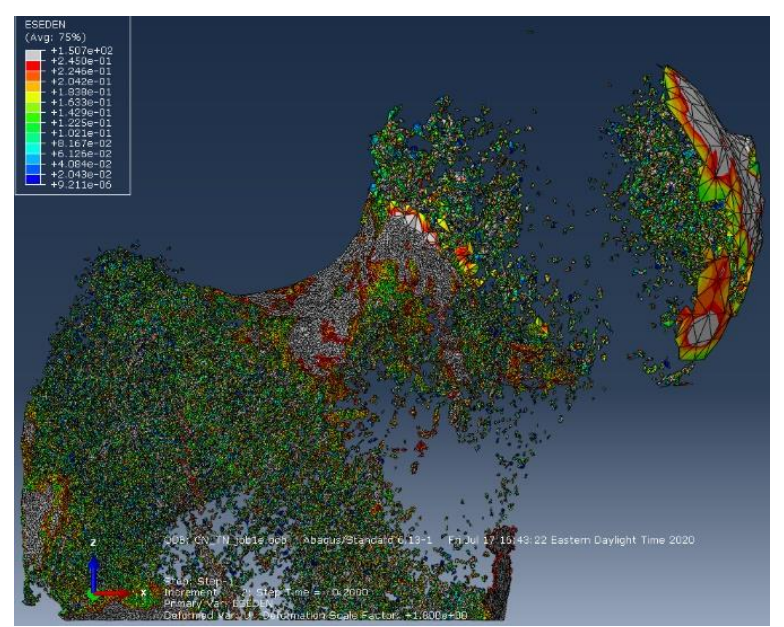

Figure 71: SED plot displays a group of elements strained at 0.245 or higher at $2000 \mathrm{~N}$ for the basic model

The Excel file listing elements strained at 0.245 or greater with their values of SED and volumes at the $2000 \mathrm{~N}$ load is included in Appendix 1.

Table 6: The volume fraction of failed tissue

\begin{tabular}{|c|c|c|}
\hline Femur Vol.(mm $\left.{ }^{3}\right)$ & Failed Vol.(mm $\left.{ }^{3}\right)$ & fail $\%$ \\
\hline 22734.27 & 945.2 & $4.2 \%$ \\
\hline
\end{tabular}

The failure criterion was used for the successive parametric analysis to calculate the failure load at which the femur would have $4.2 \%$ of volume at a SED of 0.245 (7000 $\mu \varepsilon$ effective strain) or higher.

\subsection{Failure Loads for FE Models with Altered Parameters:}

This section presents eight FE models with plotted SED for eight varied morphological parameters as well as illustrating the elements failed in excess of 0.245. The respective SED values for the displayed failed elements and the respective volumes were reported into Excel to calculate the failure load at $4.2 \%$ of volume fraction using the linear interpolation method as shown in tables 6-13. Table 14 shows the ranking of the FE models including the basic model based on failure loads and percentages of differences for each altered model relative to the basic model. 


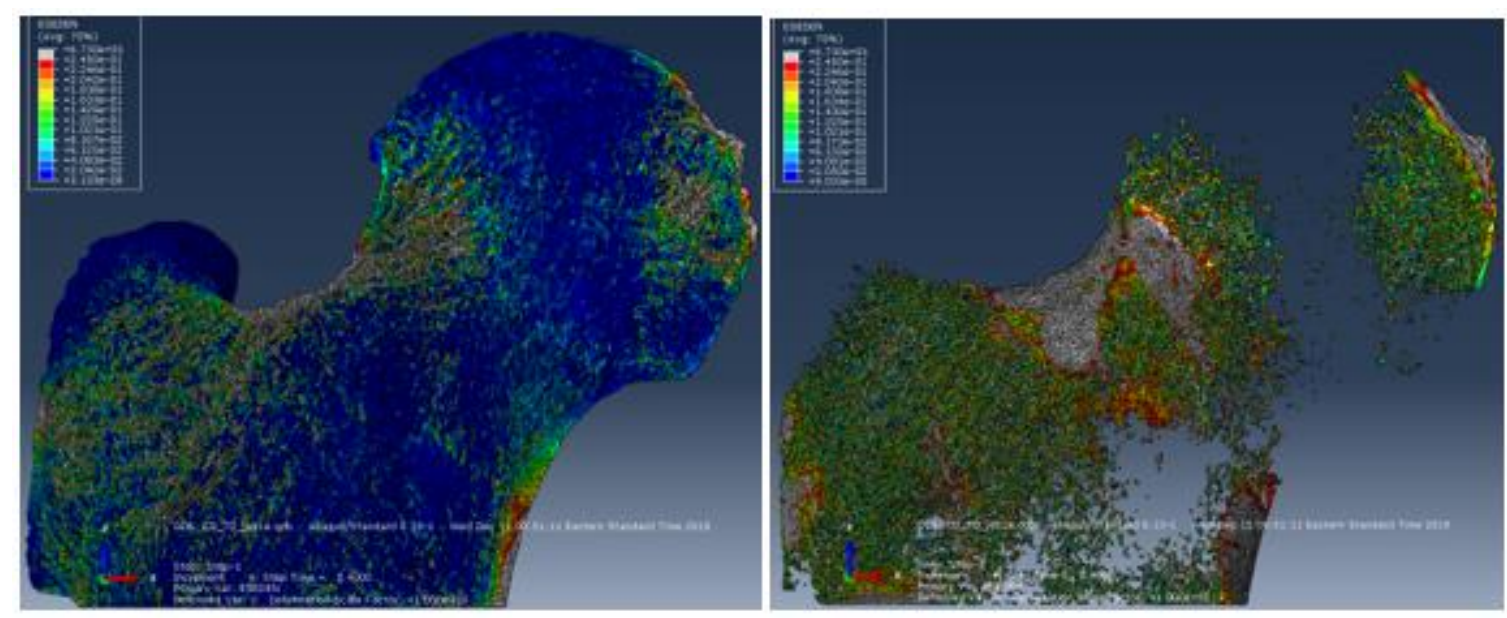

Figure 72: SED plotted at 4000N for dilated cortical and dilated trabecular bone (left). SED plot for a group of elements failed in excess of 0.245 (right)

Table 7: The failure load estimated at $4.2 \%$ using linear interpolation method for dilated both cortical and trabecular bone

\begin{tabular}{|c|c|c|c|}
\hline Load(N) & Tot_Vol $\left(\mathrm{mm}^{3}\right)$ & Fail_Vol(mm $\left.{ }^{3}\right)$ & Vol\% \\
\hline 3000 & 35416.8 & 784.3 & $2.2 \%$ \\
\hline 3625 & 35416.8 & 1487.5 & $4.2 \%$ \\
\hline 4000 & 35416.8 & 1908.5 & $5.4 \%$ \\
\hline
\end{tabular}
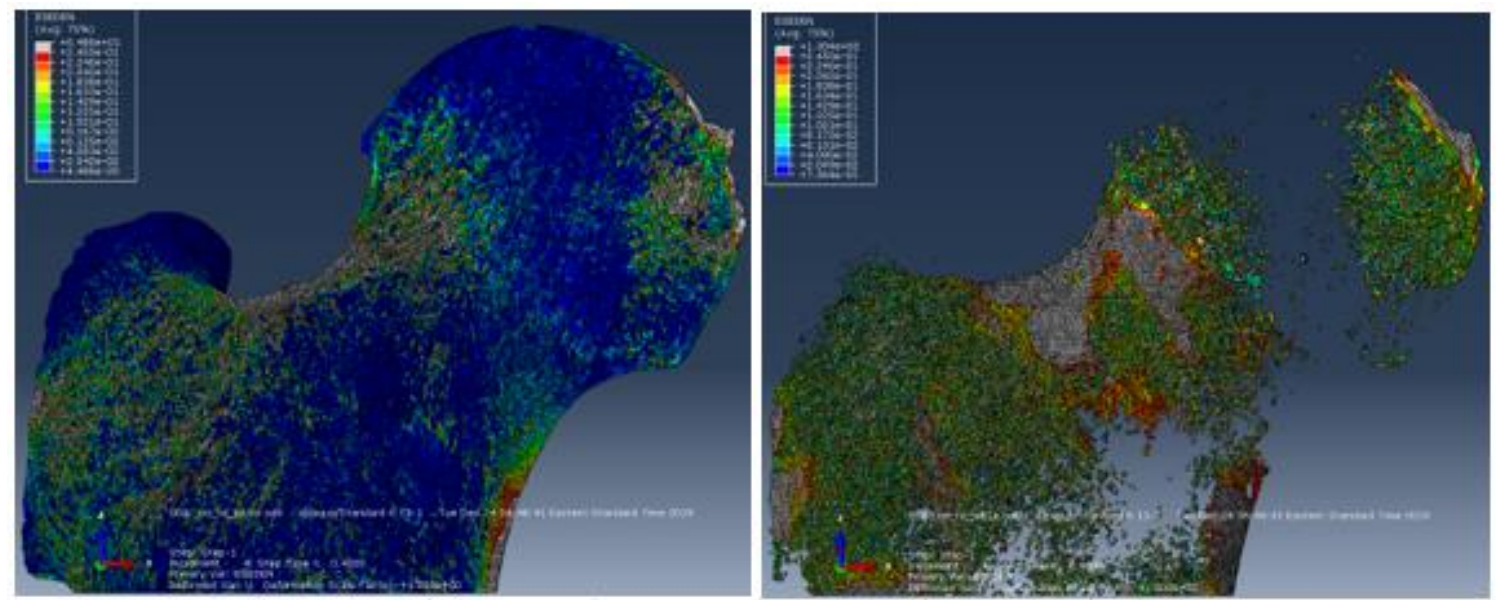

Figure 73: SED plotted at 4000N for normal cortical and dilated trabecular bone (left). SED plot for a group of elements failed in excess of 0.245 (right)

Table 8: The failure load estimated at $4.2 \%$ using linear interpolation method for normal cortical and dilated trabecular bone

\begin{tabular}{|l|c|c|c|}
\hline Load N & Total_Vol $\left(\mathrm{mm}^{3}\right)$ & Fail_Vol $\left(\mathrm{mm}^{3}\right)$ & Vol\% \\
\hline 3000 & 34988.7 & 830.6 & $2.4 \%$ \\
\hline 3564 & 34988.7 & 1469.5 & $4.2 \%$ \\
\hline 4000 & 34988.7 & 1963.9 & $5.6 \%$ \\
\hline
\end{tabular}



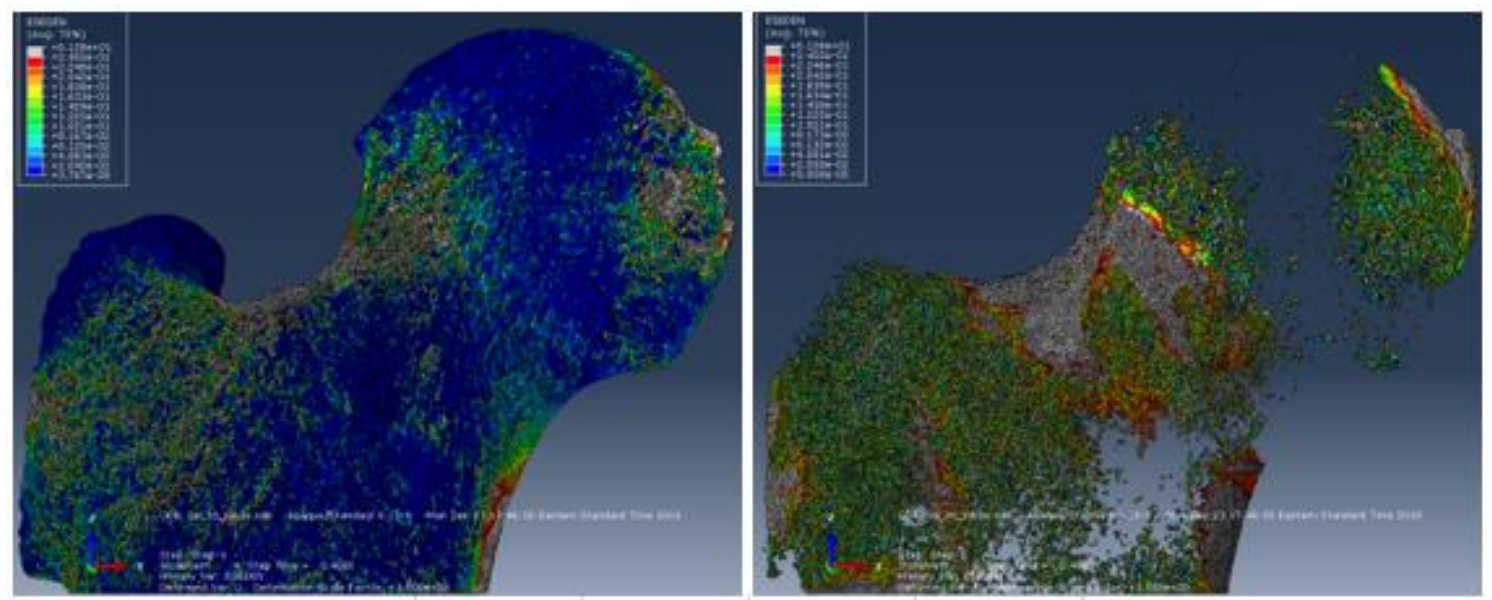

Figure 74: SED plotted at 4000N for eroded cortical and dilated trabecular bone (left). SED plot for a group of elements failed in excess of 0.245 (right)

Table 9: The failure load estimated at $4.2 \%$ using linear interpolation method for eroded cortical and dilated trabecular bone

\begin{tabular}{|l|c|c|c|}
\hline LoadN & Total_Vol $\left(\mathrm{mm}^{3}\right)$ & Fail_Vol $\left(\mathrm{mm}^{3}\right)$ & Vol\% \\
\hline 3000 & 34162.5 & 942.6 & $2.8 \%$ \\
\hline 3415 & 34162.5 & 1434.8 & $4.2 \%$ \\
\hline 4000 & 34162.5 & 2128.4 & $6.2 \%$ \\
\hline
\end{tabular}
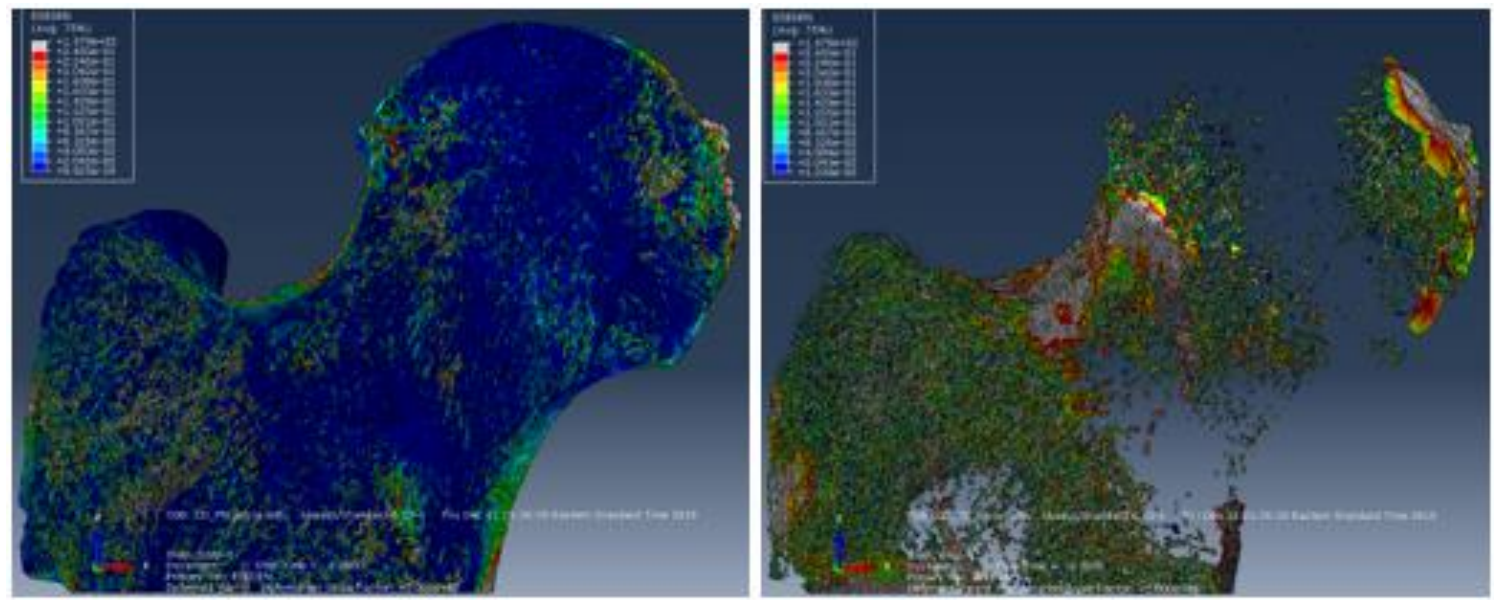

Figure 75: SED plotted at 2000N for dilated cortical and normal trabecular bone (left). SED plot for a group of elements failed in excess of 0.245 (right)

Table 10: The failure load estimated at $4.2 \%$ using linear interpolation method for dilated cortical and normal trabecular bone

\begin{tabular}{|c|c|c|c|}
\hline Load(N) & Tot_Vol(mm $\left.{ }^{3}\right)$ & Fail_Vol $\left(\mathrm{mm}^{3}\right)$ & Vol\% \\
\hline 2000 & 22966.5 & 459.4 & $2.0 \%$ \\
\hline 2298 & 22966.5 & 964.6 & $4.2 \%$ \\
\hline 3000 & 22966.5 & 2152.5 & $9.4 \%$ \\
\hline
\end{tabular}



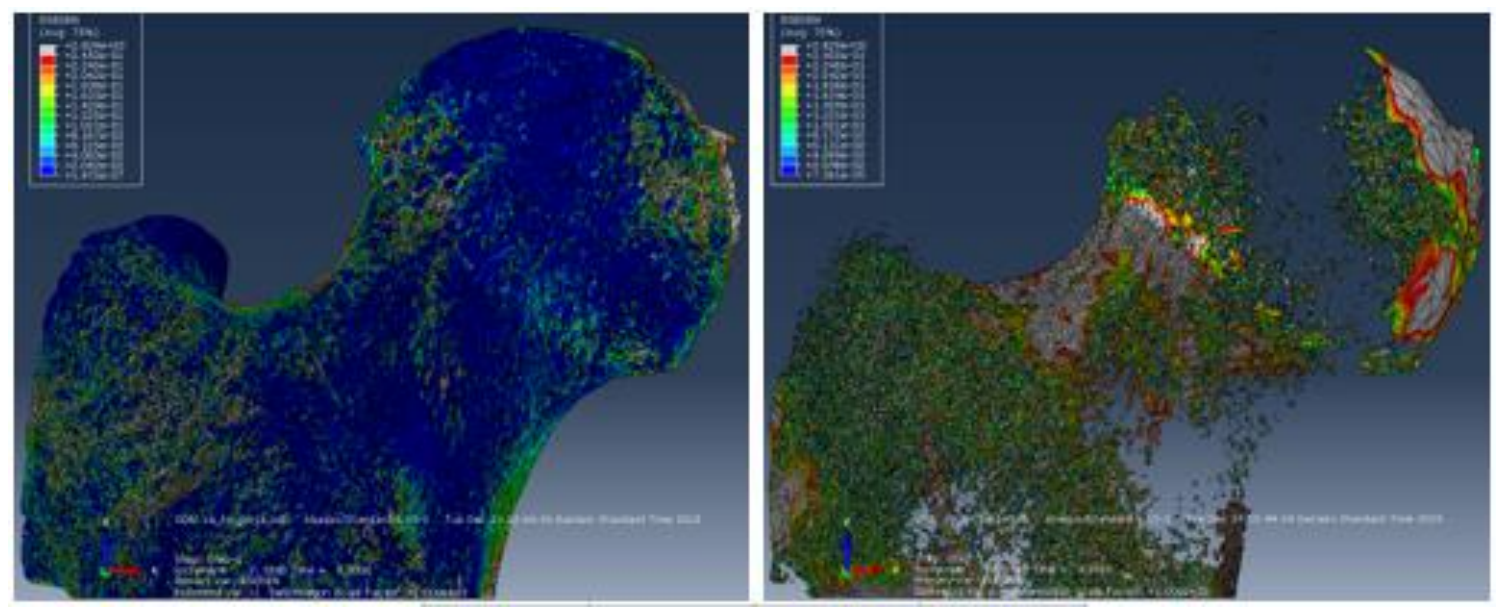

Figure 76: SED plotted at 2000N for eroded cortical and normal trabecular bone (left). SED plot for a group of elements failed in excess of 0.245 (right)

Table 11: The failure load estimated at $4.2 \%$ using linear interpolation method for eroded cortical and normal trabecular bone

\begin{tabular}{|c|c|c|c|}
\hline Load(N) & Total_Vol(mm $\left.{ }^{3}\right)$ & Fail_Vol $\left(\mathrm{mm}^{3}\right)$ & Vol $\%$ \\
\hline 1000 & 22095.7 & 103.5 & $0.5 \%$ \\
\hline 1846 & 22095.7 & 928.0 & $4.2 \%$ \\
\hline 2000 & 22095.7 & 1078.4 & $4.9 \%$ \\
\hline
\end{tabular}
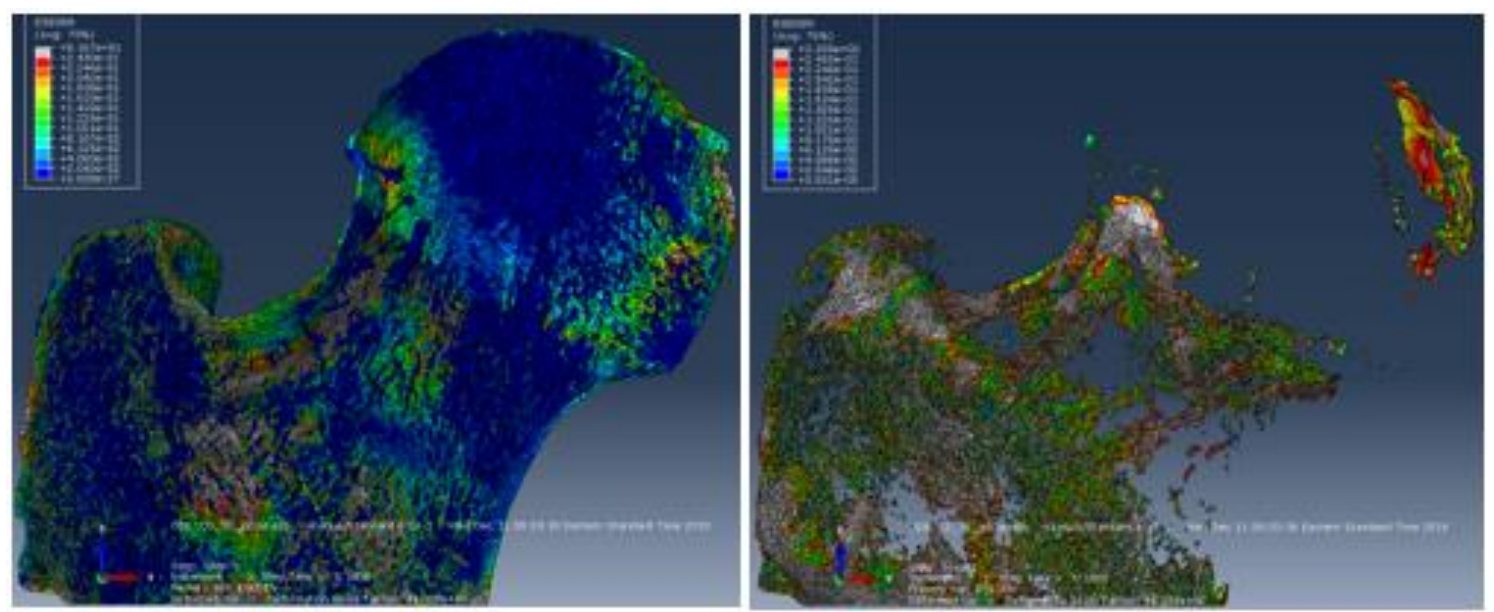

Figure 77: SED plotted at 1000N for dilated cortical and eroded trabecular bone (left). SED plot for a group of elements failed in excess of 0.245 (right)

Table 12: The failure load estimated at $4.2 \%$ using linear interpolation method for dilated cortical and eroded trabecular bone

\begin{tabular}{|c|c|c|c|}
\hline Load(N) & Tot_Vol(mm3) & Fail_Vol(mm3) & Vol\% \\
\hline 643 & 12976.2 & 545.0 & $4.2 \%$ \\
\hline 1000 & 12976.2 & 847.6 & $6.5 \%$ \\
\hline
\end{tabular}



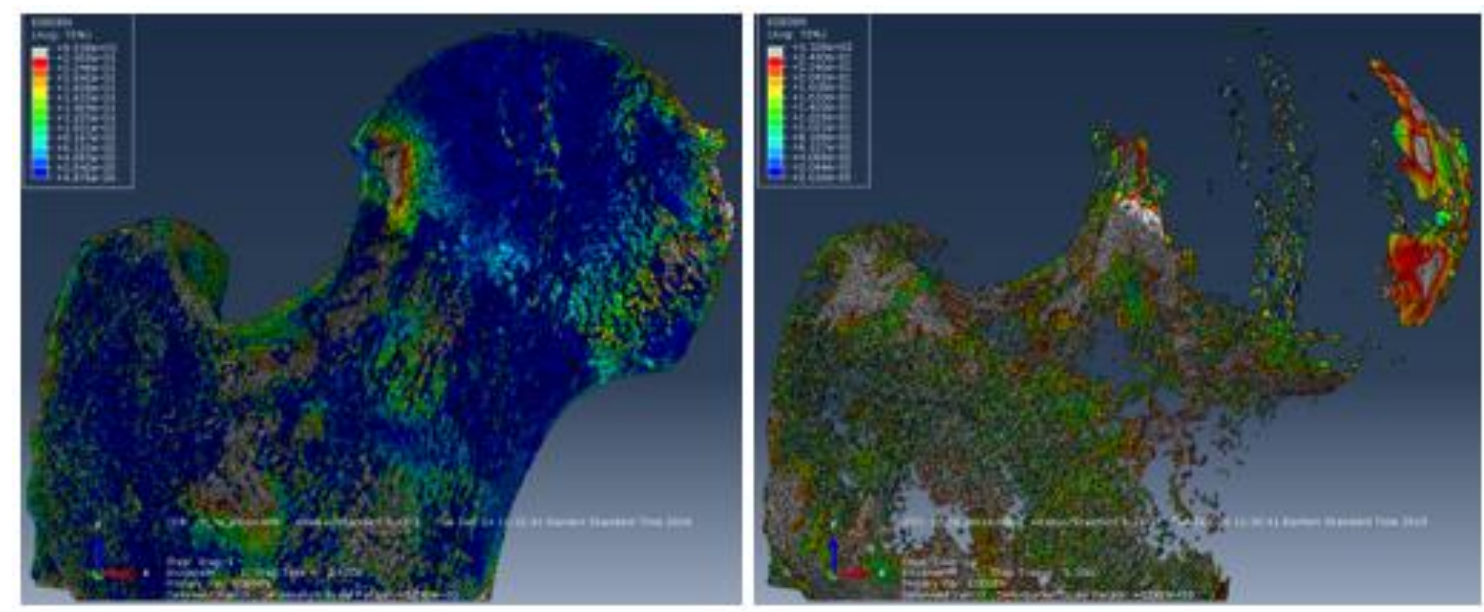

Figure 78: SED plotted at 1000N for normal cortical and eroded trabecular bone (left). SED plot for a group of elements failed in excess of 0.245 (right)

Table 13: The failure load estimated at $4.2 \%$ using linear interpolation method for normal cortical and eroded trabecular bone

\begin{tabular}{|c|c|c|c|}
\hline Load(N) & Total_Vol $\left(\mathrm{mm}^{3}\right)$ & Fail_Vol( $\left.\mathrm{mm}^{3}\right)$ & Vol\% \\
\hline 540 & 12702.2 & 533.5 & $4.2 \%$ \\
\hline 1000 & 12702.2 & 987.2 & $7.8 \%$ \\
\hline
\end{tabular}
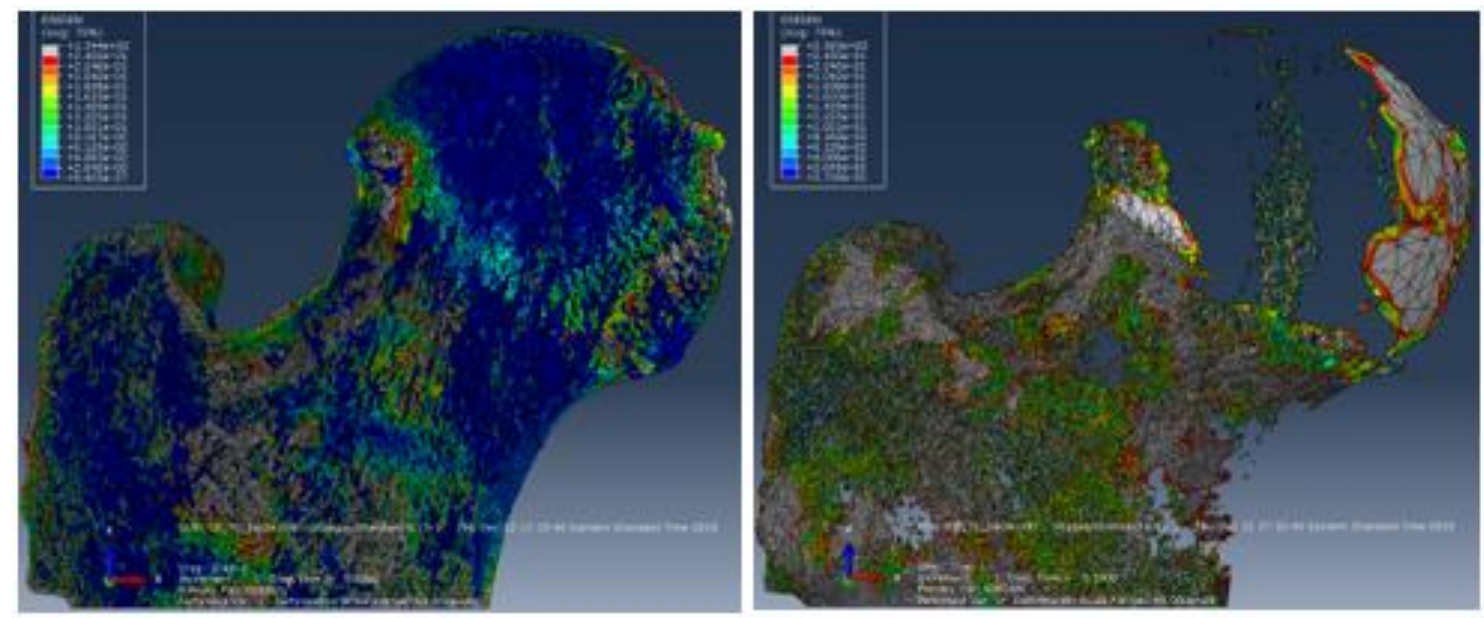

Figure 79: SED plotted at 1000N for eroded cortical and eroded trabecular bone (left). SED plot for a group of elements failed in excess of 0.245 (right)

Table 14: The failure load estimated at $4.2 \%$ using linear interpolation method for eroded cortical and eroded trabecular bone

\begin{tabular}{|c|c|c|c|}
\hline Load(N) & Tot_Vol( $\left.\mathrm{mm}^{3}\right)$ & Fail Vol $\left(\mathrm{mm}^{3}\right)$ & Vol $\%$ \\
\hline 324 & 11483.9 & 482.3 & $4.2 \%$ \\
\hline 1000 & 11483.9 & 1489.1 & $13.0 \%$ \\
\hline
\end{tabular}

By comparing the number of elements (volume of bone tissue at $4.2 \%$ ) in each

of the models in excess of SED of 0.245 , which is the accepted value of the failure 
strain energy density of bone tissue corresponding to an effective strain of $7000 \mu \varepsilon$, the estimated breaking strength of each of the models could be estimated. These results are shown in Table 14.

Table 15: FE Models Ranking Based on Failure Loads as the differences between loads and volumes were calculated

\begin{tabular}{|c||c|c||c|c|}
\hline Model & Load(N) & Difference $\%$ & Volume(mm3) & Difference $\%$ \\
\hline \hline $\begin{array}{c}\text { CortexErode } \\
\text { TrabecularErode }\end{array}$ & 324 & $-83.80 \%$ & 11483.9 & $-49.49 \%$ \\
\hline $\begin{array}{c}\text { CortexNormal } \\
\text { TrabecularEerode }\end{array}$ & 540 & $-72.98 \%$ & 12702.2 & $-44.13 \%$ \\
\hline $\begin{array}{c}\text { CortexDilate } \\
\text { TrabecularErode }\end{array}$ & 643 & $-67.85 \%$ & 12976.2 & $-42.92 \%$ \\
\hline $\begin{array}{c}\text { CortexErode } \\
\text { TrabecularNormal }\end{array}$ & 1846 & $-7.70 \%$ & 22095.7 & $-2.81 \%$ \\
\hline $\begin{array}{c}\text { CortexNormal } \\
\text { TrabecularNormal }\end{array}$ & 2000 & $0.00 \%$ & 22734.27 & $0.00 \%$ \\
\hline $\begin{array}{c}\text { CortexDilate } \\
\text { TrabecularNormal }\end{array}$ & 2298 & $14.90 \%$ & 22966.5 & $1.02 \%$ \\
\hline $\begin{array}{c}\text { CortexErode } \\
\text { TrabecularDilate }\end{array}$ & 3415 & $70.75 \%$ & 34162.5 & $50.27 \%$ \\
\hline $\begin{array}{c}\text { CortexNormal } \\
\text { TrabecularDilate }\end{array}$ & 3584 & $79.20 \%$ & 34988.7 & $53.90 \%$ \\
\hline $\begin{array}{c}\text { CortexDilate } \\
\text { TrabecularDilate }\end{array}$ & 3625 & $81.25 \%$ & 35416.8 & $55.79 \%$ \\
\hline
\end{tabular}

As expected, the results indicate that the failure strength was reduced the most when both the cortex and the trabeculae were eroded, and the failure strength was increased the most when both the cortex and the trabeculae were dilated. However, the changes in the cortex had less effect than the changes in the trabeculae. The largest effect on proximal femoral strength is realized by an increase or decrease in trabecular bone thickness. 


\section{CHAPTER 5: DISCUSSION}

Clinically, osteoporosis is usually determined from measuring the BMD using a DXA scan. Although it is referred to as the "gold standard" by the World Health Organization, it is limited in predicting hip fracture risk [69]. Therefore, scientific and clinical efforts have expanded to include more sophisticated methods to determine bone quality and to include bone microstructure in an attempt to create more accurate hip fracture risk assessments. The current study attempts to shed light on the influence of incremental thickening or thinning of the bone structural components of the proximal femur using a combination of experimental tests and FEA [69]. The following sections describe various results from previous studies utilizing these two approaches either alone or in combination and discusses similarities and differences between their work and the present study.

\subsection{Experimental studies:}

There have been numerous studies that specifically measured the breaking strength of human cadaver femurs [69]. Of these, 24 studies tested female femurs subjected to sideways fall configuration. The overall average fracture force was 3262 $\mathrm{N}$, with a range of 573-15,034 $\mathrm{N}$.

One of the studies included 34 female cadaver femora, with ages of $81.6 \pm 10.7$ years, mechanically tested in side impact configuration after they were scanned by DXA and radiographed using "Faxitron" X-ray system [70]. The X-ray system provided both trabecular bone structure and geometry. The study aimed: a) to apply a 
gradient based image processing method for the assessment of trabecular structure, density and bone geometry from projectional radiographs, b) to compare the radiographic results with the BMD determined by DXA, and c) to test whether the measured strength of femur can be estimated from radiographs by combining analyses of its trabecular structure and geometry. The measured mean failure load for the cervical (femoral neck) fractures was equal to $2,879 \mathrm{~N} \pm 1,117 \mathrm{~N}$ in women ( $\mathrm{n}=30$ ). In trochanteric fracture cases, Euler number and femoral cortex thickness explained 66\% of the variability in failure load, while trochanteric BMD explained 72\%. In cervical fracture cases, trabecular bone area and femoral neck axis length explained $64 \%$ of the variability in failure loads, while femoral neck BMD also explained 64\%. the study showed that combined analysis of trabecular bone structure and geometry predicts in vitro failure load with similar accuracy as DXA.

Another large cadaver experimental study included 77 left female cadaveric femora with an average age of 81.7 years [71]. The objective was to investigate whether there exist differences in the distribution of cervical and trochanteric fractures between different structural mechanical strength levels and different sexes and to identify the geometric determinants that predict a fracture type. The bones were radiographed with a Faxitron X-rays, which were digitized together with a calibration scale using a scanner with a resolution of 900 dpi. The neck-shaft angle, femoral shaft cortex width, calcar femoral cortex width, femoral neck axis length, femoral head and neck diameters, trochanteric width, and femoral shaft diameter were measured. The authors broke the results into quartiles based on the failure loads and performed statistical analysis on the quartile groups. The study reported at the lowest load quartiles, 94.7\% 
of fractures in female and $62.5 \%$ in males were femoral neck fractures. Throughout the four quartiles, the failure load ranged from $\leq 2297.5 \mathrm{~N}$ to $>3563.5 \mathrm{~N}$. It concluded that femoral neck fractures predominate at the lowest structural mechanical strength levels. Therefore, females are more susceptible to femoral neck fractures than males and the female femoral neck fracture represents the lowest failure load range for all hip fractures caused by a fall to the side. This further confirms that the results presented here for a single female femur are in an expected and appropriate range.

The cadaver femur in the current study failed at a load of $2530 \mathrm{~N}$ and had an initial failure at $2140 \mathrm{~N}$ when loaded in a fall-to-the-side configuration. Considering the donor was a female aged 68 years, this failure strength appears to be in a range expected based on other similar studies. In addition, it was not surprising that the femur fractured at the femoral neck and not the trochanter.

\subsection{FE Convergence:}

FE Convergence is a method of demonstrating the mathematical accuracy of FEA. From the literature, it has been observed that results of FEA can be shown to converge to an exact solution by two methods: $\mathrm{p}$-convergence and h-convergence. The h-convergence method, or as it is called, mesh-refinement, is when the number of elements is increased by reducing the element size. Whereas, the p-convergence method is when the mathematical order of the element type is increased, which is accomplished by increasing the number of nodes in each element without necessarily changing the number of elements. This gives each element more degrees of freedom, because, for example, the sides of an element represented by three nodes can assume a curved shape whereas an element side represented by only two nodes must form a 
straight line. The resolution in the present model had to be reduced from $60-\mu \mathrm{m}$ to $120-$ $\mu \mathrm{m}$ of element size due to limitations of number of triangles in Mimics and also computational limitations due to memory restrictions. Therefore, an abbreviated form of the p-convergence method was used in ABAQUS to demonstrate that the present model trended toward convergence. The displacement of a specified reference node number 1128312 located on the upper lateral edge of the femoral head under a load of 2000-N for two identical meshes of the same model: one a mesh using 4-noded elements, and the other a mesh using 10-noded elements. Although this reference node displaced considerably more in the 10-noded element mesh than in the 4-noded element mesh at $2000 \mathrm{~N}$, we expect that displacement of the reference would node rapidly converge to an exact solution if it was possible to create meshes with still more DOF (higher number of nodes than the 10-node model). Therefore, the 10-noded element mesh was considered adequate and used for all of the subsequent FE models.

\subsection{FE Validation Studies:}

To better understand bone mechanical properties and to validate numerical models, reliable and relevant data from experimental tests should be obtained and compared to the models [72]. Strain measurement is one of the most efficient representatives for interpreting experimental data for validating computational modeling. Experimental strain values have been measured using several techniques with substantial differences in terms of accuracy, precision, time, and length scale. When designing an experiment, each technique involves pros and cons that must be carefully evaluated. Furthermore, when applying such strain measurement techniques to bone, extra complexities are often encountered due to its complex bio-composite 
structure. The extra complexities are mainly due to the irregular geometry, surface roughness and moisture. One of the most commonly adopted methods for strain measurement is through the use of electrical resistance foil strain gauges. In fact, foil strain gauges are considered the gold standard in bone strain measurement because of their accuracy and high response [72] when utilized in a relatively short timeframe so gauge performance does not deteriorate.

The strain gauge approach was used by a group out of Washington University in St. Louis to study animal bones of different ages. Female mice tibiae at 5,12, and 22 months of age were subjected to axial compression loading to study strain-adaptive (re)modeling [73]. While specimen-specific finite element analysis was used to examine strain distribution along the tibial length, a three-gauge experimental method was used to determine the strain distribution at the mid-diaphysis. The three-gauge method allowed to determine the neutral axis of bending, the line along which strain equals zero at the mid-diaphysis. The gauges' locations were determined based on both the consistent position observed by micro-CT and the force-strain linearity at each site. Axial strain was the main outcome from the FE analysis, from which the average strain values corresponding to the strain gauges were calculated. Baseline scans were registered with scans taken with gauges attached to identify the exact gauge locations on the FE models. A component labeling tool was used for locations of each gauge site, which were segmented from the baseline scans and identified on the FE model. A MATLAB program was used to segment periosteal elements corresponding to the surface area of each gauge site. The study calculated the average for each gauge surface and later compared them with the experimental strain values. An axial compression 
applied between the ankle and knee produced a combination of compression plus bending at the mid-diaphysis that resulted in a gradient from compression to tension due to the curvature of the tibia along its length. The experimental and numerical results involved 36 to 45 specimens from which the average values were computed. The percentage of differences of the FE strains relative to the experimental strains for the five-month specimens ranged from $8 \%$ to $16 \%$, for the twelve-month specimens ranged from $9 \%$ to $48 \%$, and for the twenty-two-month specimens ranged from $11 \%$ to $42 \%$. From these results, the average value of percentage of difference for the entire results was $\sim 25 \%$, whereas the percentages of difference for the current study are $2.1 \%$ at $1000 \mathrm{~N}$ and $9 \%$ at $2000 \mathrm{~N}$. The higher percentage of difference of $9 \%$ is acceptable, only if the strain values in the region immediately under the strain gauge were within the elastic region, whereas strain values at somewhere else on the femoral neck were higher or exceeded the yielding strain value.

Two substantial concepts were used in common in this study and the current study: using the measured strains to validate the calculated FE resultant strains. One of the noticeable differences is that study used the strain gauges approach for the mice tibiae, whereas the current study used it for analyses of a subject-specific proximal femur.

Subject-specific FE modeling is the only technique to estimate bone stresses non-invasively in-vivo [74]. Although many studies used this technique, few of them obtained acceptable accuracy in predicting bone strength relative to the measured strength. Several issues that resulted in inaccurate FE models were addressed. Automatic mesh generation has been criticized since it was applied over whole bones 
especially at anatomical regions with complex geometry or higher curvatures. Low accuracy was observed in FE models meshed with voxel-based meshes since this strategy generates jagged edges on the bone surface. Critical issues were presented in the epiphysis and metaphysis regions of FE models since difficulties were involved to define the distribution of the material properties. Even though some studies described bone as a homogeneous two-phase material with defined material properties of cortical and trabecular bone, they either lacked the experimental validation or did not investigate the differences in the stress field distribution in the whole bone. Therefore, great attention has been given to investigate up to which extent of accuracy the CTbased FE modeling could predict the mechanical stresses induced by physiological activities in human bones. CT-based FE models were automatically generated for a human right proximal femur, 51-year-old male donor, which was tested in-vitro under five different scenarios [74]. The study aimed to estimate the FE application accuracy in predicting the measured experimental data. The study used pre-wired stacked rosette strain gauges for thirteen locations over femoral head, neck, diaphysis, and metaphysis. A rosette strain gauge consists of two or more strain gauges that are positioned closely to measure strains along different directions of the component under evaluation. Single strain gauges can only measure strain effectively in one direction, so the use of multiple strain gauges enables more measurements to be taken, providing a more precise evaluation of strain on the surface being measured. The principal stresses were determined for the comparison with the FE results by collecting the maximum and minimum principal strain values from the 13 rosettes for the highest load $(800 \mathrm{~N})$ and $30 \mathrm{~s}$ after load application in each loading scenario. Two FE models were generated for 
each experimental scenario: a density-based FE model, and a simplified two-material FE model which was later verified to check how would it change the accuracy obtained from the density-based FE model. The stresses were measured and computationally calculated based on measured and calculated strains. Although the two-material model showed higher root mean square errors (RMSE), range of 0-35\% and average of 9.6 $\%$, than the density-based model errors, range of $0-26 \%$ and average of $8.6 \%$, its results are still in an acceptable level. The percentage of difference in the current study is $2.1 \%$ which is less than their averaged RMSE of $8.6 \%$. Although the study used values of stresses for validation, it is OK for using strain values for validation in the current study since both studies used linear analysis for FE modeling. Furthermore, the stress was measured in their experiments based on the measured strains as the stress is computationally independent since the strain is dependent on the modulus of elasticity.

\subsection{Failure Criteria:}

The strain threshold value of $7000 \mu \varepsilon$ was used as the failure criterion for the current study. It was converted to the corresponding SED value of 0.245 since it could readily be reported from ABAQUS.

The same strain threshold value of $7000 \mu \varepsilon$ was used previously for studying a human radius $[48,62]$. Those results revealed that the calculated tissue-level strains can be significantly better predictor of bone fracture load $\left(\mathrm{R}^{2}=0.75\right)$ than bone mass $\left(\mathrm{R}^{2}<0.48\right)$ or structural indices $\left(\mathrm{R}^{2}<0.57\right)$. The best agreement was found where $\mathrm{R}^{2}=0.75$ as $2 \%$ of the bone tissue strained beyond the yield strain at the onset of fracture. Although the current study shows $4.2 \%$ of the failed bone tissue, each result is acceptable only for the respective study with subject-specific parameters. The reason 
that the $2 \%$ of the radius study showed the best correlation with the radius strength could be for example the total size of the radius used in FE modeling is larger than that of the current FE model, or the failure of the radius occurred locally where the area of failed elements is very small relative to that in the current FE model.

Similar failure criterion of effective strain was used by Nawathe et al (2014) for understanding the microstructural failure mechanisms of the human proximal femur during a sideways fall impact. Microstructural failure mechanisms of twelve human proximal femora were simulated and mechanically broken in the sideways fall loading configuration [67]. The initial structural failure of the femur was determined in FE simulations when the failure of a small amount of the bone tissue (1.5\% to $6.4 \%$ across all bones) was observed. The proportion of failed tissue, estimated by the finite element models, decreased with decreasing measured femoral strength [67]. Although there are several differences, there are several similarities such as model volume that has exceeded a certain strain. In their study, the percentage of the FE model volume was calculated based on the valid correlation between the directly measured yield strength and the FE predicted one.

In the current study however, the percentage of the failed FE femoral bone tissue volume (exceeding $7000 \mu \varepsilon$ ) for the basic model was calculated based on the SED plot from which each element with SED value exceeding 0.245 was reported at loading level of $2000 \mathrm{~N}$. Later, that same percentage (4.2\%) was used to predict failure load for each of the remaining FE models using interpolation method within loading levels which have percentages above and below $4.2 \%$. This approach, and in particular the failure criteria used in the current study, appears to be acceptable because it is 
consistent with and similar to results found in other previous studies. Not only does the amount of bone tissue exceeding the predetermined yield level fall within the same range as those found by other research groups, but the failure loads for a range of different models (as discussed in the next section) match up well with the ranges of failure loads seen in other experimental and FEA studies of hip fractures due to fall-tothe-side loading.

The elements that were exposed directly to boundary and loading conditions were eliminated from reporting the group of elements that exceeded $7000 \mu \varepsilon$. This was necessary because the actual load transfer through the joint medially and the soft tissues laterally could not be perfectly replicated, and it was observed in Abaqus that those locations experienced very high strains. It is further justified to exclude these regions from the fracture analysis because clinically and experimentally, fractures rarely if ever occur in these regions of the proximal femur.

\subsection{Overall FE Results:}

As expected, the current study reveals higher or lower predicted FE failure loads than that of the basic FE model $(2000 \mathrm{~N})$ due to thickening or thinning trabecular or cortical bone. The calculated failure loads ranged from 324 to $3625 \mathrm{~N}$ with the mean value of $2031 \mathrm{~N}$. The corresponding FE bone tissue volumes range from 11,484 to $35,417 \mathrm{~mm}^{3}$ with the mean value of $23,281 \mathrm{~mm}^{3}$.

Many other studies predicted FE failure loads for mechanically fractured human cadaveric proximal femora due to sideways falling configuration [22, 64, 67, 75-77]. Briefly, these studies: estimated the amount and location of internal tissue-level failure, validated FE strains and fracture locations, predicted fracture type of trochanteric vs. 
neck fracture, determined the association between radiation-free MRI-derived bone strength and strain simulations compared to results from direct mechanical testing, and estimated the contributions of femoral neck cortical and trabecular bone to proximal femur failure load.

An improved understanding of the microstructural failure mechanisms of the human proximal femur during a sideways fall impact might elucidate the etiology of hip fracture [67]. The estimated failure loads for the twelve femurs reported in the study by Nawathe et al., (2014), ranged from 930 to $4670 \mathrm{~N}$, whereas the estimated failure loads for the nine FE models in the current study ranged from 324 to $3625 \mathrm{~N}$. The volumetric BMD of each FE model in each study was estimated as illustrated in Fig. 79. As noticed from the figure, each trendline has excellent correlation and demonstrates a strong linear relationship between bone tissue density and failure strength. Their data and the data from the present study have different slopes due to several reasons. Their vBMD was calculated based on the femoral neck only, whereas vBMD calculated for the models in this study included the femoral head and trochanter, which is expected to result in higher vBMD because the regions outside the femoral neck tend to have more trabecular bone tissue. They used actual DXA scan to measure areal $\mathrm{BMD}$, while the $\mathrm{vBMD}$ calculation for the models in that study used a general bone density value of $1.8 \mathrm{~g} / \mathrm{cm}^{3}$ for normal bone. 


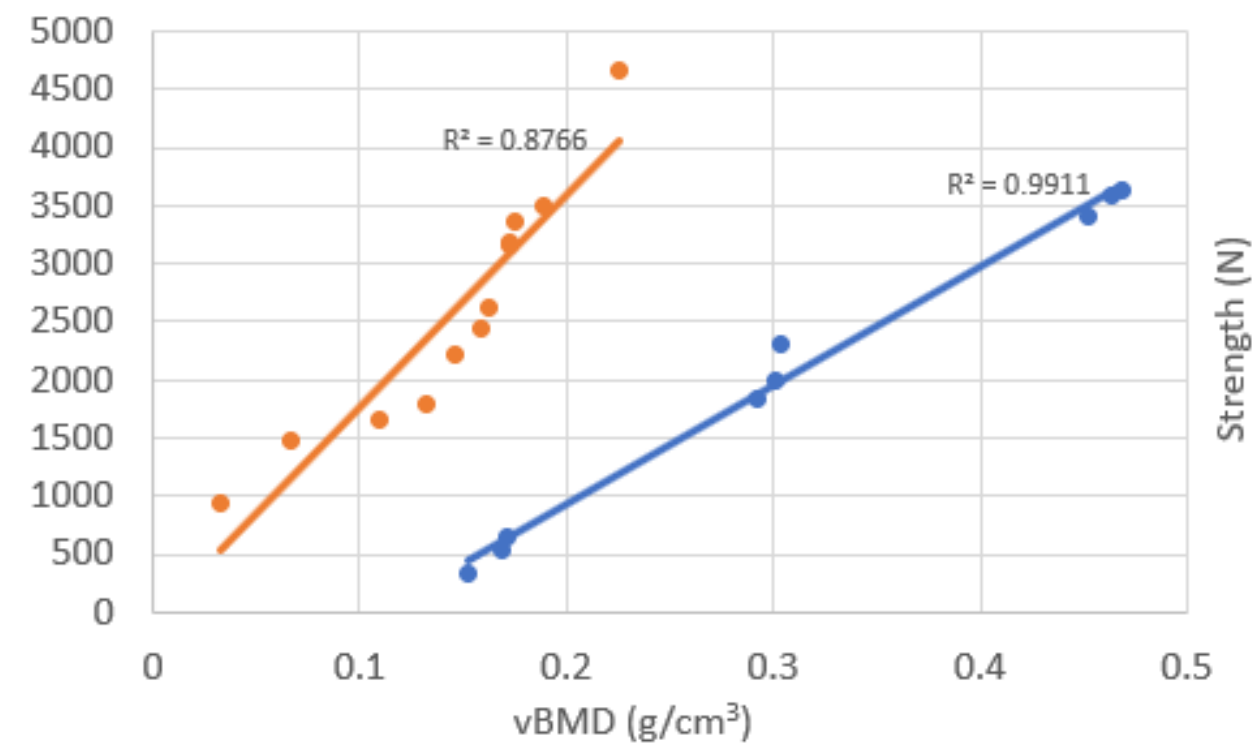

Figure 80: Strength vs volumetric bone mineral density (vBMD) for the current study (blue) and the study of the 12 femurs (red). The trend line of each study is illustrated

Nevertheless, both studies demonstrated powerful linear correlations between measures of the overall amount of bone tissue material available to carry the load of the falling impact and the strength of the proximal femur (load at which it failed). In most respects, the FE results in the current study are very consistent with that estimated for the twelve femurs [67].

Several other studies with similar experimental and computational modeling approaches reported consistent measured and estimated failure loads. Eight female femora, aged $76 \pm 10$ years, were mechanically fractured as linearly modeled using FEA for sideways fall loading to estimate the amount and location of internal tissue-level failure [67]. The mean value of experimental yield strength was $2590 \mathrm{~N}$, whereas the FEA estimated strength was $2690 \mathrm{~N}$ ranged from 930 to $4670 \mathrm{~N}$. In the same way of mechanical testing and FE modeling, ten female femora, aged $66.5 \pm 14.5$ years, were tested to investigate whether the type of fracture is closely related to the patient-specific femoral structure and predictable by FE methods [77]. Results revealed the mean value of the measured fracture load was $2619 \mathrm{~N}$ ranged from 1151 to $4166 \mathrm{~N}$, whereas the 
mean value of the FE load was 2901 N ranged from 1190 to 3154 N. Six female cadaveric femurs with average age of 76 years were mechanically tested with a hydraulic universal test frame to simulate loading in a sideways fall orientation [75]. The study aimed to determine the association between radiation-free MRI-derived bone strength and strain simulations compared to results from direct mechanical testing of cadaveric femora. The study reported an average failure load of 3300 N. Considerably, findings in these studies and those in the current study are consistent.

Findings in the aforementioned studies and the current study demonstrate that the significance of different parameters for the estimation of bone strength is dependent on both the anatomical site and the mechanical loading.

Some previous studies investigated whether the BMD was a predictor of hip fracture $[67,78]$. A group of elderly women included 167 healthy women, 24 women with trochanteric, and 42 women with cervical hip fracture were involved in a study to investigate whether their measured BMDs in specific regions of the lower and upper part of the femoral neck on DXA scans were predictors of the type of hip fracture [79]. Results revealed that prediction of femoral neck fracture was enhanced by measuring only the upper part of the femoral neck BMD, as the lower part BMD was not different from that of the healthy women.

Human cadaveric femurs (48 women and 25 men, aged 74.2 \pm 8.7 years, range 55-98 years) were obtained as their femoral neck (FN) aBMDs were measured by DXA, and were mechanically tested to failure in a sideways fall configuration [78]. The force applied to the hip during a sideways fall was estimated from height and weight and accounted for trochanteric soft tissue thickness. Among those diagnosed 
with osteoporosis by femoral neck aBMD, 98\%. Results revealed that aBMD is a significant predictor for femurs with measured $\mathrm{T}$-scores $<-2.5$, which would be vulnerable to femoral neck fractures.

BMD was measured for a sample that included 8 female proximal femurs aged $76 \pm 10$ years to check whether it is relevant to the bone tissue proportion in the femoral neck region [67]. Results revealed that aBMD is well correlated with low measured strength.

\subsection{The Importance of Interaction and Relative Role of the Trabeculae Versus Cortical Bone in the Strength of Bony Structure:}

Diagnosis of osteoporosis may be improved and insight into the effect of disease, aging, and drug treatment may be provided as well when the biomechanical role of the cortical shell with respect to the trabecular bone has been accurately understood. The improved understanding of these biomechanical roles may also lead to successful validation of FE models for fracture risk prediction and improved design of bone implants.

A compressive loading was applied upon a 26-year-old male cadaveric distal radius to evaluate which structural parameters (other than bone mass) are the best predictors for changes in the radius mechanical properties after different bone atrophy scenarios [62]. Furthermore, the study evaluated whether the original strength of the affected bone can be recovered if bone loss is restored by thickening of the remaining structures. The full three-dimensional architecture of the distal radius was extracted at an isotropic resolution of $80-\mu \mathrm{m}$ using a micro-CT scanner. The mechanical variations due to various modified bone structure were studied using micro-FE simulations, from which eight models were created. Four current models with mass lowered by $20 \%$, 
either by reducing cortical thickness, trabecular thickness, number of trabeculae, or by overall thinning of structures. The bone mass was restored to the original value using a trabecular bone thickening procedure in the other four current models. Analyses revealed that cortical bone carried most of the load. Therefore, bone strength was affected most in the reduced cortical thickness model. For the same reason, all the trabecular bone atrophy scenarios affected bone strength in a very similar way and resulted in less dramatic bone strength reduction. The original bone strength was not recovered when the bone mass was restored. It is noteworthy that the cortical bone in this study is more important than the trabecular bone, which is opposite with results of the current study.

The trabecular load distribution was evaluated in an osteoporotic and a healthy vertebra under normal daily loads and collateral loads with combination of HRCT of entire bones, $\mu$-FEA, and parallel supercomputers [33]. Surprisingly, the number of highly loaded trabeculae in the osteoporotic vertebra was not significantly different from that in the healthy one under normal daily loads ( $8 \%$ and $9 \%$ respectively). Significant bone loss is pronounced in the osteoporotic trabeculae which compensated its effects with more orientation in the longitudinal direction, and thus ensuring adequate stiffness for normal daily loading. The osteoporotic structure was made less resistant against collateral "error" loads as a result of increased orientation. In the case or collateral loading, the number of overloaded trabeculae in the healthy vertebra was lower than in the osteoporotic one ( $4 \%$ and $13 \%$, respectively). These findings confirm the relationship between bone morphology and the external loads applied during normal daily life. 
The effects of removal of the shell from the vertebral body and the associated mechanical effects in terms of overall change in vertebral structural stiffness and of the tissue-level stresses were presented using FE simulations [63]. Specimen-specific FE models of thirteen vertebrae were generated from $\mu$-CT scans with $60-\mu \mathrm{m}$ voxel size. Compressive loading conditions were applied after removal of the endplates, and linear elastic analysis were run for three cases: with and without the shell, and shell only models. As the study indicated that the calculated FE results were consistent with other previous experimental studies. Results indicated that the shell was on average, $0.38 \pm$ $0.06 \mathrm{~mm}$ thick, accounted for $21-39 \%$ of the overall bone mass, but accounted for 38 $68 \%$ of the overall vertebral stiffness. When the tissue-level stresses were examined, it was inferred that this disproportionately large mechanical effect of shell removal was due in part to unloading of the remaining peripheral trabeculae adjacent to the shell. Stress was preferentially incurred within vertically aligned bone: the cortical shell and vertically aligned trabeculae. Two important roles of the thin vertebral cortical shell were demonstrated by these results: it can carry significant load by virtue of representing a large proportion of the vertically aligned bone tissue within the vertebra, and, as a shell, it also maximizes the load carrying capacity of the trabecular centrum particularly around the periphery.

\subsection{Hip Fracture Prevention Procedure:}

No significant differences were observed between the fracture and control groups in regards of comparing the periosteal expression of the bone formation marker alkaline phosphate for the femoral neck [80]. A histological study noticed a relatively slow turnover occurring at the periosteum of ilium bone [81]. These findings suggest 
that cortical shell reduction may be highly dependent on a remodeling deficit occurring at the endocortical-marrow interface [82]. Bone fragility at endocortical-marrow interface may be strongly influenced by bone turnover events at this skeletal site, since cortical shell thinning is a feature of intracapsular hip fracture in elderly female subjects [83].

\subsection{Limitations:}

The current study could obtain better results if it did not have the following limitations. Unavailability of a DXA scanner prevented comparing the current results more directly to one or more of the results from the literature. The femur in the current study was modeled as isotropic, whereas the real bone tissue is anisotropic. Modeling the femur with isotropic property was supported by a study that had bone cubes from the spine, the calcaneus, the distal, and the proximal femur. They were tested multi directionally to measure their compressive stiffness and to calculate their ratios of mechanical anisotropy [32]. The ultimate strength was determined in a destructive test in the vertical direction. Anisotropy ratios in the vertical direction were larger at the distal femur and the spine than in the proximal femur. The predictive power of BMD for the mechanical properties varied with anatomical location and strongly depended on the loading direction. The correlations of mechanical properties with BMD were greatest at locations where the anisotropy was less pronounced (proximal femur) [32]. The predetermined experimental falling "impact" velocity of $100 \mathrm{~mm} / \mathrm{s}$ is generally different from the real impact velocity because of limitations in the speed with which laboratory testing machines can operate. Also, it is not possible to know every parameter that can be determined to influence the fall force, such as body weight, body 
height, and trochanteric soft tissue thickness. The most accurate approach to obtain these parameters is to derive them experimentally from real human bodies, which is not possible with a destructive way and especially for the impact velocity [69]. The precise determination of the strain gauge location for the FE models was another challenging procedure which involved some error. The proper method to accurately predict the bone fracture strength is to consider a nonlinear elasto-plastic property for FE models. However, the linear elastic property was chosen for the current study because of the available technology hardware, memory, and software specifications. The reduction in the scanning resolution was imposed by the technology limitations, which prevented the study from producing all bone structural details. Furthermore, the sizes of FE models were limited in MIMICS for number of triangles, which may lead to additional errors. All results are specific for the current study because only one proximal femur was tested and modeled. The current study could be made better by repeating the same work with several different femurs of different age, bone density, etc. to see if the results hold for other femurs. Even though the above limitations are true, the current study at least tested a very appropriate femur (Female age 68 ) for this area of study (osteoporosis related hip fractures).

It may have been more appropriate to extend the inferior free surface of the model using a coarsened element structure in order to provide a more similar boundary condition between the model and the experiment. If this was done, the strains observed in the model at the location of Strain Gauge 4 would likely have been in closer agreement because the proximity of the gauge site to the boundary constraints would not have had such an influence on local strains. However, this was not done because 
of the difficulty within ABAQUS of transitioning between such a fine mesh on the distal surface and the center of rotation more distally in the femur shaft. The mesh density and the total number of degrees of freedom of the model were already near the computational limits of the hardware and software available at the time of this project, so expanding the model was not attempted. 


\section{CHAPTER 6: CONCLUSION}

A practical and precise procedure for modeling a human cadaveric proximal femur was developed and verified. Although it was necessary due to computational limitations to reduce the modeling resolution from $60 \mu \mathrm{m}$ to $120 \mu \mathrm{m}$, the FE basic model was shown to be accurate and validated by agreement of calculated results with the experimental measurements. The systematic parametric variation in morphological parameters showed that the trabecular bone is more significant than the cortical bone regarding femoral neck strength. Increasing all of the proximal femoral trabeculae by approximately $120 \mu \mathrm{m}$ (which represents an increase in proximal femoral BMD of 53.9\%) results in a $79.2 \%$ increase in the failure strength under fall-to-the-side loading. Similarly, decreasing the trabecular thickness by the same amount reduces the failure strength by $72.98 \%$. Thus, the current study may be potentially advantageous in future research when the micro-CT based FE mechanism becomes directly applicable to patient-specific analysis. Likewise, these results can guide current clinical practice to focus on strategies that target increasing the trabecular thickness of the bone in the femoral neck. 


\section{REFERENCES}

1. Jacobsen, S.J., et al., Hip fracture incidence among the old and very old: $a$ population-based study of 745,435 cases. American journal of public health, 1990. 80(7): p. 871-873.

2. Blume, S.W. and J.R. Curtis, Medical costs of osteoporosis in the elderly Medicare population. Osteoporosis International, 2011. 22(6): p. 1835-1844.

3. Surgeons, A.A.o.O., Study Outlines Risk Factors for Poor Outcome, Mortality Following Hip Fracture. 2013.

4. Bass, E., et al., Risk-adjusted mortality rates of elderly veterans with hip fractures. Annals of epidemiology, 2007. 17(7): p. 514-519.

5. General, O.o.t.S., Bone health and osteoporosis: a report of the Surgeon General. 2004.

6. Viswanathan, H.N., et al., Direct healthcare costs of osteoporosis-related fractures in managed care patients receiving pharmacological osteoporosis therapy. Applied health economics and health policy, 2012. 10(3): p. 163-173.

7. Burge, R., et al., Incidence and economic burden of osteoporosis-related fractures in the United States, 2005-2025. Journal of bone and mineral research, 2007. 22(3): p. 465-475.

8. Health, N.I.o., Osteoporosis prevention, diagnosis, and therapy. NIH consensus statement, 2000. 17(1): p. 1-36.

9. Kanis, J.A., Diagnosis of osteoporosis and assessment of fracture risk. The Lancet, 2002. 359(9321): p. 1929-1936.

10. Foundation, N.O. 54 Million Americans Affected by Osteoporosis and Low Bone Mass. 20142014 11/24/2020]; Available from: https://www.nof.org/news/54million-americans-affected-by-osteoporosis-and-low-bone-mass/.

11. Marks, R., et al., Hip fractures among the elderly: causes, consequences and control. Ageing research reviews, 2003. 2(1): p. 57-93.

12. Greenspan, S.L., et al., Fall severity and bone mineral density as risk factors for hip fracture in ambulatory elderly. Jama, 1994. 271(2): p. 128-133.

13. Berry, S.D. and R.R. Miller, Falls: epidemiology, pathophysiology, and relationship to fracture. Current osteoporosis reports, 2008. 6(4): p. 149-154.

14. Freedman, K.B., et al., Treatment of osteoporosis: are physicians missing an opportunity? JBJS, 2000. 82(8): p. 1063.

15. Giangregorio, L.M., et al., Too Fit To Fracture: exercise recommendations for individuals with osteoporosis or osteoporotic vertebral fracture. Osteoporos Int, 2014. 25(3): p. 821-35.

16. Raef, H., et al., Updated recommendations for the diagnosis and management of osteoporosis: a local perspective. Ann Saudi Med, 2011. 31(2): p. 111-28. 
17. Cowin, S.C., Bone Mechanics. 1989: CRC-Press.

18. Osunderu, A.O., BASIC ANATOMY AND PHYSIOLOGY OF HUMAN BODY.pdf. 2008, National Open University of Nigeria.

19. Zimmerman, M. and B. Snow, An_Introduction_to_Nutrition.pdf. 2012.

20. Jones, O., <proximal femur.pdf>. 2013.

21. Grewal, S.W.M., <Human biology.pdf>. 2020.

22. Verhulp, E., B. van Rietbergen, and R. Huiskes, Load distribution in the healthy and osteoporotic human proximal femur during a fall to the side. Bone, 2008.

42(1): p. 30-35.

23. Caeiro, J., P. González, and D. Guede, Biomechanics and bone (\& II): Trials in different hierarchical levels of bone and alternative tools for the determination of bone strength. Rev Osteoporos Metab Miner, 2013. 5(2): p. 99-108.

24. Knudson, D.V., Fundamentals of biomechanics. 2007, Springer: New York.

25. KARDUNA, A.R., <biomechanical principles.pdf>.

26. Soudah, E., et al., Numerical Methods and Applications in Biomechanical Modeling 2014. Comput Math Methods Med, 2015. 2015: p. 768652.

27. Alexander, R.M., Mechanics of animal movement. Current biology, 2005. 15(16): p. R616-R619.

28. Keaveny, T.M., et al., Biomechanics of trabecular bone. Annual review of biomedical engineering, 2001. 3(1): p. 307-333.

29. Oftadeh, R., et al., Biomechanics and mechanobiology of trabecular bone: a review. Journal of biomechanical engineering, 2015. 137(1).

30. OpenStax, <Exercise, Nutrition, Hormones, and Bone Tissue.pdf>. 2013.

31. Clinic, C., <Osteoporosis_clevelandclinicmeded.pdf〉. 2010.

32. Augat, P., et al., Anisotropy of the elastic modulus of trabecular bone specimens from different anatomical locations. Medical engineering \& physics, 1998. 20(2): p. 124-131.

33. Homminga, J., et al., The osteoporotic vertebral structure is well adapted to the loads of daily life, but not to infrequent "error" loads. Bone, 2004. 34(3): p. 510516.

34. Hopkinson-Woolley, J.A. and M.J. Parker, Fractures of the hip: does the type of fall really affect the site of fracture? Injury, 1998. 29(8): p. 585-587.

35. Lotz, J., E. Cheal, and W. Hayes, Stress distributions within the proximal femur during gait and falls: implications for osteoporotic fracture. Osteoporosis International, 1995. 5(4): p. 252-261.

36. Pinilla, T.P., et al., Impact Direction from a Fall Influences the Failure Load of the Proximal Femur as Much as Age-Related Bone Loss. Calcified Tissue International, 1996. 58(4): p. 231-235.

37. Pulkkinen, P., et al., Association of geometric factors and failure load level with the distribution of cervical vs. trochanteric hip fractures. J Bone Miner Res, 2006. 21(6): p. 895-901.

38. Adams, A.L., et al., Osteoporosis and hip fracture risk from routine computed tomography scans: the fracture, osteoporosis, and CT utilization study (FOCUS). Journal of Bone and Mineral Research, 2018. 33(7): p. 1291-1301. 
39. Bouxsein, M., B. Coan, and S. Lee, Prediction of the strength of the elderly proximal femur by bone mineral density and quantitative ultrasound measurements of the heel and tibia. Bone, 1999. 25(1): p. 49-54.

40. Ulrich, D., et al., The ability of three-dimensional structural indices to reflect mechanical aspects of trabecular bone. Bone, 1999. 25(1): p. 55-60.

41. Center, N.-D.T.R. <History of Radiography.pdf>. Available from: https://www.nde-ed.org/AboutNDT/aboutndt.htm.

42. Hendee, W.R., Cross sectional medical imaging: a history. Radiographics, 1989. 9(6): p. 1155-1180.

43. BASE, W.S., <Computed Tomography history.pdf>. 2013.

44. Krug, R., et al., High-resolution imaging techniques for the assessment of osteoporosis. Radiologic Clinics, 2010. 48(3): p. 601-621.

45. Geusens, P., et al., High-resolution in vivo imaging of bone and joints: a window to microarchitecture. Nature Reviews Rheumatology, 2014. 10(5): p. 304-313.

46. Kinney, J., N.E. Lane, and D. Haupt, In vivo, three-dimensional microscopy of trabecular bone. Journal of Bone and Mineral Research, 1995. 10(2): p. 264-270.

47. Kabel, J., et al., Connectivity and the elastic properties of cancellous bone. Bone, 1999. 24(2): p. 115-120.

48. Pistoia, W., et al., Estimation of distal radius failure load with micro-finite element analysis models based on three-dimensional peripheral quantitative computed tomography images. Bone, 2002. 30(6): p. 842-848.

49. Rauf, P., A Brief Review of Image Segmentation Techniques. IJCSIS, 2017. 15(4).

50. Varma, D.R., Managing DICOM images: Tips and tricks for the radiologist. The Indian journal of radiology \& imaging, 2012. 22(1): p. 4.

51. Kato, A. and N. Ohno, Construction of three-dimensional tooth model by microcomputed tomography and application for data sharing. Clinical oral investigations, 2009. 13(1): p. 43-46.

52. MATERIALISE, <Materialise Mimics_3D Medical Image Processing Software.pdf>.

53. MATERIALISE, <Mimics 14.12 Reference Guide.pdf>. 2011.

54. Chepelev, L., et al., Medical 3D printing for vascular interventions and surgical oncology: a primer for the 2016 radiological society of North America (RSNA) hands-on course in 3D printing. 3D printing in medicine, 2016. 2(1): p. 1-17.

55. Jagota, V., A.P.S. Sethi, and K. Kumar, Finite element method: An overview. Walailak Journal of Science and Technology (WJST), 2013. 10(1): p. 1-8.

56. Papadopoulos, P., <Introduction to the Finite Element Method.pdf>. 2015.

57. Papadopoulos, P., <Introduction to the Finite Element Method.pdf>. 2015.

58. Bathe, K.-J.r., Finite element procedures. Second edition. ed. 2014, [United States]: [Klaus-Jürgen Bathe].

59. Logan, D., <Mesh generation and assembly ch 5.pdf>, in A First Course in the Finite Element Method. 2016, Cengage Learning

60. MIT. <Eight-node brick element (C3D8 and F3D8).pdf>. 2014.

61. Cook, R.D., Concepts and applications of finite element analysis. $2 \mathrm{~d}$ ed. ed. 1981, New York: Wiley. 
62. Pistoia, W., B. Van Rietbergen, and P. Rüegsegger, Mechanical consequences of different scenarios for simulated bone atrophy and recovery in the distal radius. Bone, 2003. 33(6): p. 937-945.

63. Eswaran, S.K., et al., The micro-mechanics of cortical shell removal in the human vertebral body. Computer methods in applied mechanics and engineering, 2007. 196(31-32): p. 3025-3032.

64. Nawathe, S., et al., Cortical and trabecular load sharing in the human femoral neck. Journal of biomechanics, 2015. 48(5): p. 816-822.

65. Holdsworth, D.W. and M.M. Thornton, Micro-CT in small animal and specimen imaging. Trends in Biotechnology, 2002. 20(8): p. S34-S39.

66. Techniques, T. Wheatstone Bridge - Load Cell Manufacture.pdf. 2020; Available from: https://www.transducertechniques.com/wheatstone-bridge.aspx.

67. Nawathe, S., et al., Microstructural failure mechanisms in the human proximal femur for sideways fall loading. Journal of bone and mineral research, 2014. 29(2): p. 507-515.

68. de Bakker, P.M., et al., During sideways falls proximal femur fractures initiate in the superolateral cortex: evidence from high-speed video of simulated fractures. Journal of biomechanics, 2009. 42(12): p. 1917-1925.

69. Sarvi, M.N. and Y. Luo, Sideways fall-induced impact force and its effect on hip fracture risk: a review. Osteoporosis international, 2017. 28(10): p. 2759-2780.

70. Pulkkinen, P., et al., Experimental hip fracture load can be predicted from plain radiography by combined analysis of trabecular bone structure and bone geometry. Osteoporosis International, 2008. 19(4): p. 547-558.

71. Pulkkinen, P., et al., Association of geometric factors and failure load level with the distribution of cervical vs. trochanteric hip fractures. Journal of Bone and Mineral Research, 2006. 21(6): p. 895-901.

72. Grassi, L. and H. Isaksson, Extracting accurate strain measurements in bone mechanics: A critical review of current methods. Journal of the mechanical behavior of biomedical materials, 2015. 50: p. 43-54.

73. Patel, T.K., M.D. Brodt, and M.J. Silva, Experimental and finite element analysis of strains induced by axial tibial compression in young-adult and old female C57Bl/6 mice. Journal of biomechanics, 2014. 47(2): p. 451-457.

74. Taddei, F., et al., Subject-specific finite element models of long bones: an in vitro evaluation of the overall accuracy. Journal of biomechanics, 2006. 39(13): p. 2457-2467.

75. Rajapakse, C.S., et al., MRI-based assessment of proximal femur strength compared to mechanical testing. Bone, 2020. 133: p. 115227.

76. Liebl, H., et al., In-vivo assessment of femoral bone strength using Finite Element Analysis (FEA) based on routine MDCT imaging: a preliminary study on patients with vertebral fractures. PloS one, 2015. 10(2): p. e0116907.

77. Ridzwan, M.I.Z., et al., Femoral fracture type can be predicted from femoral structure: a finite element study validated by digital volume correlation experiments. Journal of Orthopaedic Research®, 2018. 36(3): p. 993-1001.

78. Roberts, B.J., et al., Comparison of hip fracture risk prediction by femoral aBMD to experimentally measured factor of risk. Bone, 2010. 46(3): p. 742-746. 
79. Duboeuf, F., et al., Different morphometric and densitometric parameters predict cervical and trochanteric hip fracture: the EPIDOS Study. Journal of bone and mineral research, 1997. 12(11): p. 1895-1902.

80. Power, J., et al., Evidence for bone formation on the external" periosteal" surface of the femoral neck: a comparison of intracapsular hip fracture cases and controls. Osteoporosis international, 2003. 14(2): p. 141-145.

81. Balena, R., M.S. Shih, and A.M. Parfitt, Bone resorption and formation on the periosteal envelope of the ilium: a histomorphometric study in healthy women. Journal of Bone and Mineral Research, 1992. 7(12): p. 1475-1482.

82. Bell, K., et al., Structure of the femoral neck in hip fracture: cortical bone loss in the inferoanterior to superoposterior axis. Journal of Bone and Mineral Research, 1999. 14(1): p. 111-119.

83. Power, J., et al., Bone remodeling at the endocortical surface of the human femoral neck: a mechanism for regional cortical thinning in cases of hip fracture. Journal of Bone and Mineral Research, 2003. 18(10): p. 1775-1780. 


\section{APPENDIX}

\section{Appendix A:}

\begin{tabular}{|c|c|c|c|c|c|c|c|c|}
\hline & & EVOL & 1938471 & 61.9246 & $2.34 \mathrm{E}-03$ & 185687 & $2.45 \mathrm{E}-01$ & $2.20 \mathrm{E}-03$ \\
\hline 2001940 & 258.261 & $2.00 \mathrm{E}-04$ & 1804486 & 60.1706 & $1.39 \mathrm{E}-04$ & 175326 & $2.45 \mathrm{E}-01$ & $2.49 \mathrm{E}-03$ \\
\hline 2066140 & 237.734 & $31 \mathrm{E}-03$ & 158291 & 58.963 & $2.52 \mathrm{E}-03$ & 1650229 & $2.45 \mathrm{E}-01$ & 4.37E-03 \\
\hline 108875 & 137.618 & $51 \mathrm{E}-03$ & 2209184 & 56.5467 & $1.31 \mathrm{E}-06$ & 2114911 & $2.45 \mathrm{E}-01$ & $1.32 \mathrm{E}-03$ \\
\hline 1547665 & 137.271 & $6.19 \mathrm{E}-04$ & 2152601 & 56.4397 & $1.17 \mathrm{E}-03$ & 1609464 & $2.45 \mathrm{E}-01$ & $2.72 \mathrm{E}-03$ \\
\hline 2217416 & 134.438 & $53 \mathrm{E}-04$ & 1893912 & 52.9657 & $1.66 \mathrm{E}-03$ & 92254 & $2.45 \mathrm{E}-01$ & $3.61 \mathrm{E}-03$ \\
\hline 1702863 & 133.182 & 4.92E-04 & 2060720 & 51.2169 & $1.65 \mathrm{E}-03$ & 1194264 & $2.45 \mathrm{E}-01$ & $5.42 \mathrm{E}-03$ \\
\hline 641155 & & $11 \mathrm{E}-04$ & 2195635 & 49.2834 & $1.02 \mathrm{E}-03$ & 1760177 & $2.45 \mathrm{E}-01$ & $E-03$ \\
\hline 2152618 & 131. & $81 \mathrm{E}-03$ & & 49.2138 & 4.77E-03 & 1268169 & $2.45 \mathrm{E}-01$ & $2.29 \mathrm{E}-03$ \\
\hline 2155393 & & $3.80 \mathrm{E}-03$ & 2216525 & 49.1417 & $3.56 \mathrm{E}-04$ & & & \\
\hline & & & 215 & & $1.85 \mathrm{E}-03$ & 1107345 & $2.45 \mathrm{E}-01$ & $1.89 \mathrm{E}-03$ \\
\hline 1991579 & 107.487 & $2.04 \mathrm{E}-03$ & & 178 & $=-04$ & 772281 & $2.45 \mathrm{E}-01$ & \\
\hline & & & 1329139 & 46.9693 & 7.47E-04 & 65405 & $E-01$ & -04 \\
\hline 1192798 & 992 & $53 \mathrm{E}-03$ & & & E-03 & 1879547 & $2.45 \mathrm{E}-01$ & $E-03$ \\
\hline 1669909 & 99.0962 & $2.43 \mathrm{E}-03$ & 2073705 & 46.6813 & $E-04$ & & & \\
\hline & & & 219 & 46.0723 & $2.12 \mathrm{E}-03$ & 394040 & $2.45 \mathrm{E}-01$ & $3.41 \mathrm{E}-03$ \\
\hline 1702932 & 95.7095 & 91E-04 & 2190874 & & $=-04$ & & & \\
\hline 1328930 & 24 & $21 \mathrm{E}-04$ & 1929001 & 44.2971 & $7.23 \mathrm{E}-04$ & 786665 & $2.45 \mathrm{E}-01$ & $E-03$ \\
\hline & & & 199 & & $E-04$ & 1340383 & -01 & $E-03$ \\
\hline 1904343 & 80.096 & $31 \mathrm{E}-04$ & 2129943 & 43.9274 & 7.57E-04 & & & \\
\hline 51120 & & $58 \mathrm{E}-04$ & 2132517 & 43.5607 & 1.17E-03 & 426518 & $.45 E-01$ & $1.83 \mathrm{E}-02$ \\
\hline 1638803 & 72.2749 & 13E-04 & 2205 & & 4.34E-06 & & -01 & $=-03$ \\
\hline 923510 & 412 & $1.32 \mathrm{E}-03$ & 2171801 & 43.1046 & $6.71 \mathrm{E}-04$ & 1101739 & $2.45 \mathrm{E}-01$ & $4.23 \mathrm{E}-03$ \\
\hline 2145947 & 67.3072 & $3.14 \mathrm{E}-04$ & 2040869 & 42.8206 & $1.40 \mathrm{E}-03$ & 18764 & $2.45 \mathrm{E}-01$ & 4.77E-03 \\
\hline 1844328 & 67.1137 & 7.13E-04 & 383766 & 41.7036 & $1.83 \mathrm{E}-04$ & 1469984 & $2.45 \mathrm{E}-01$ & 4.49E-03 \\
\hline 2042719 & 63.6191 & $6.50 \mathrm{E}-04$ & 2100428 & 41.1587 & $6.78 \mathrm{E}-04$ & 1283229 & $2.45 \mathrm{E}-01$ & $4.28 \mathrm{E}-03$ \\
\hline 1719021 & 3.0184 & $90 \mathrm{E}-03$ & 1519622 & 41.0676 & $6.24 \mathrm{E}-04$ & 411217 & $2.45 \mathrm{E}-01$ & $3.61 \mathrm{E}-03$ \\
\hline 1938471 & 61.9246 & 2.34E-03 & 2066934 & 40.667 & 4.09E-04 & 889259 & $2.45 \mathrm{E}-01$ & $1.79 \mathrm{E}-03$ \\
\hline 1804486 & 60.1706 & $1.39 \mathrm{E}-04$ & 2049534 & 40.6013 & $1.40 \mathrm{E}-04$ & 2011149 & $2.45 \mathrm{E}-01$ & $3.84 \mathrm{E}-03$ \\
\hline 158291 & 58.963 & $2.52 \mathrm{E}-03$ & 2090458 & 40.4317 & $3.09 E-04$ & 1198521 & $2.44 \mathrm{E}-01$ & $1.84 \mathrm{E}-03$ \\
\hline
\end{tabular}

\section{Appendix B:}

*Heading

** Job name: ce_te_job1e Model name: ce_te

** Generated by: Abaqus/CAE 6.13-1

$*$ Preprint, echo $=\mathrm{NO}$, model $=\mathrm{NO}$, history $=\mathrm{NO}$, contact $=\mathrm{NO}$ 


\section{** PARTS}

*Part, name $=$ CE_TE

*Node

$1, \quad 6.73922491, \quad 40.3481636,0.0276999995$

$2, \quad 6.96606445, \quad 41.4388046,0.0276999995$

$3, \quad 8.7002449, \quad 42.5408287,0.0278999992$

4, 4.70989847, 39.6692696, 0.0280000009

$5, \quad 5.87301874, \quad 40.7301407,0.0280000009$

$6, \quad 8.01053238,41.7908745,0.0282000005$

$7, \quad 1.85085261,40.3113937,0.0284000002$

$8, \quad 9.62924194,44.7641144,0.0284000002$

$9, \quad 2.49488568, \quad 38.9834442,0.0285999998$

$10, \quad 5.30641365, \quad 39.3041191,0.0285999998$

4206451, $-32.5535431,57.7793045, \quad 19.5568504$

$4206452,-37.2396507, \quad 53.4950485, \quad 25.519001$

$4206453,-37.2652473, \quad 53.9278717,26.9113007$

$4206454,-37.2706566, \quad 53.882225,26.8131485$

$4206455,-37.2387314,53.8309937,26.9738998$

$4206456,-31.6416245,41.0827065,30.0351505$

$4206457,-31.4489727,40.8271942,30.6192513$

$4206458,-30.7193451,38.2111435,25.3265991$

$4206459,-31.358429, \quad 40.3122406, \quad 29.712101$

$4206460,-32.1213608, \quad 38.7463875,25.0611496$

*Element, type $=\mathrm{C} 3 \mathrm{D} 10$

1, 541542, 541543, 541544, 541545, 700784, 700783, 700782, 700786, 700785, 700787

2, 541543, 541542, 541546, 541547, 700784, 700789, 700788, 700791, 700790, 700792

3, 541548, 541549, 541547, 541550, 700795, 700794, 700793, 700797, 700796, 700798

4, 541548, 541551, 541552, 541553, 700801, 700800, 700799, 700803, 700802, 700804

5, 541548, 541554, 541551, 541545, 700806, 700805, 700801, 700808, 700807, 700809

6, 541548, 541542, 541554, 541545, 700811, 700810, 700806, 700808, 700786, 700807

7, 541555, 541554, 541556, 541545, 700814, 700813, 700812, 700815, 700807, 700816

8, 541555, 541557, 541558, 541559, 700819, 700818, 700817, 700821, 700820, 700822

$9,541555,541545,541560,541551,700815,700824,700823,700825,700809,700826$

10, 541555, 541558, 541551, 541561, 700817, 700827, 700825, 700829, 700828, 700830

2223298, 80821, 79918, 79205, 79214,1767798,2659801,3841297,1777164,1777165,4025467

2223299, 672904, 280980, 283312, 285057,2550650,4191484,1792259,4187183,4187184,4191862

2223300, 672905, 291387, 287652, 285705,2803342,2803343,2803339,2238067,3549577,4199432

2223301, 662963, 282303, 281295, 277936,3238679,3586015,2955606,1625638,3731476,2955607

2223302, 364545, 368580, 363941, 365150,4206457,4199097,3893472,3893468,4203630,3893473

2223303, 285098, 286577, 290205, 287998,4206458, 957563,4187546,3710793, 957558, 957562

2223304, 618966, 279965, 276257, 280388,2454408,2454426,2454422,2454410,3457012,3878030

$2223305,351005,355418,355010,354708,3285070,4203656,4206459,3285068,3285066,3285235$

2223306, 370138, 365713, 366280, 366804,1888584,1888588,1888586,4203620,3563873,4206379

2223307, 277936, 282303, 281295, 281855,3731476,3586015,2955607,4202457,4007416,4206460

*Elset, elset=Bone, generate

$1,2223307, \quad 1$

*Nset, nset=Load

394792, 405968, 412672, 423260, 433888, 441866, 445303, 449118, 451215, 453846, 453864, 455610, 455983, 456624, 456998, 457109 
458143, 458457, 460282, 460518, 460751, 461062, 461155, 461494, 461611, 463342, 464516, 464889, 465181, 465328, 466429, 466523

468969, 470170, 470594, 470972, 472856, 474868, 476273, 477166, 477965, 478678, 479045, 479823, $480073,481427,482410,482851$

483182, 483245, 483433, 483442, 483655, 483753, 484110, 484227, 485039, 485405, 485834, 485942, 486061, 486237, 486558, 486908

487387, 487880, 488383, 488521, 488624, 488873, 489348, 489354, 489691, 491062, 491228, 491861, 492964, 493220, 493599, 493959

495565, 496285, 497644, 497898, 498145, 499223, 500328, 500521, 500844, 501036, 502249, 503043, $503051,503535,503777,503837$

503844, 503876, 504162, 505262, 505358, 505383, 505415, 505557, 506619, 507254, 507269, 507278, $507860,508623,508631,509005$

509105, 509171, 509296, 509852, 510047, 510223, 510282, 510299, 510594, 510651, 510683, 510931, $702314,714571,714573,714575$

725977, 727060, 733360, 850163, 875401, 875402, 875403, 879147, 888508, 888511, 888512, 933767 , 933768, 933770, 941242, 994964

$995044,1007813,1059320,1059321,1076036,1076037,1076038,1082089,1082090,1082091,1083163,1083$ $165,1083167,1084597,1084601,1084945$

\footnotetext{
*Nset, nset=T

16174, 16188, 16791, 17388, 17763, 18266, 18386, 18622, 18727, 19261, 19829, 19890, 20251, 20328, 20588, 20794

20849, 20968, 21069, 21298, 21692, 21783, 21872, 22017, 22192, 22506, 22837, 22875, 23253, 23641, 23880,24007

24111, 24236, 24691, 24782, 24947, 25055, 25101, 25565, 25575, 25812, 26089, 26306, 26397, $26552,26554,26568$

26790, 27026, 27246, 27264, 27329, 27601, 27939, 27957, 27997, 28050, 28289, 28560, 28611 , $28625,28783,28934$

29205, 29562, 29805, 29992, 30079, 30245, 30671, 30682, 30953, 30965, 30969, 31156, 31389, $31512,31542,31792$

31852, 32023, 32110, 32291, 32314, 32547, 32617, 32841, 32941, 32966, 33097, 33482, 33550, $33614,33630,33641$

$33676,33755,33834,33971,34051,34338,34687,34761,35024,35071,35504,35740,35756$, $35780,35866,36006$

$36016,36123,36817,36848,36906,36965,37010,37320,37329,37580,37713,38105,38168$, 38201, 38304, 38429

38445, 38698, 38743, 38784, 38926, 38936, 39091, 39231, 39395, 39592, 39660, 40081, 40215, 40533, 40629, 40755

40940, 41357, 41376, 41416, 41472, 41557, 41675, 41689, 41928, 42011, 42159, 42370, 42398, 42460, 42471, 42507

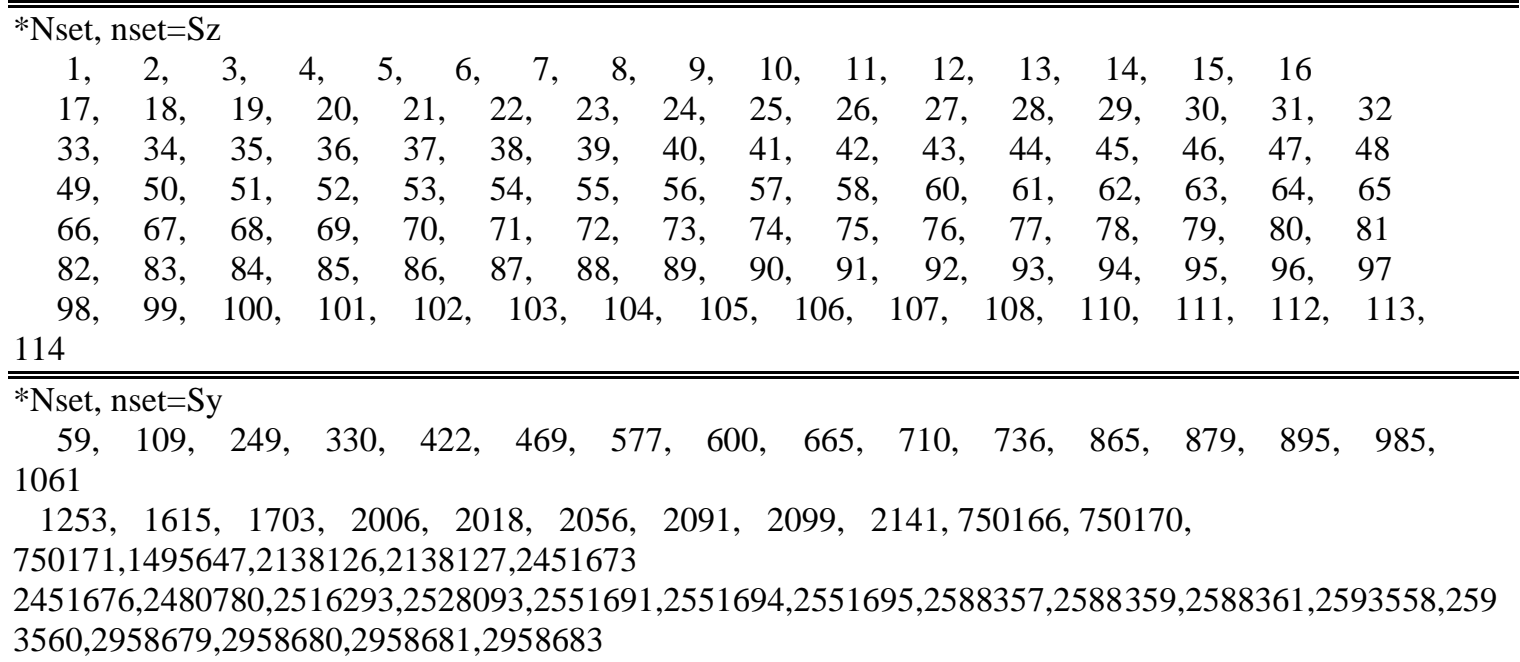




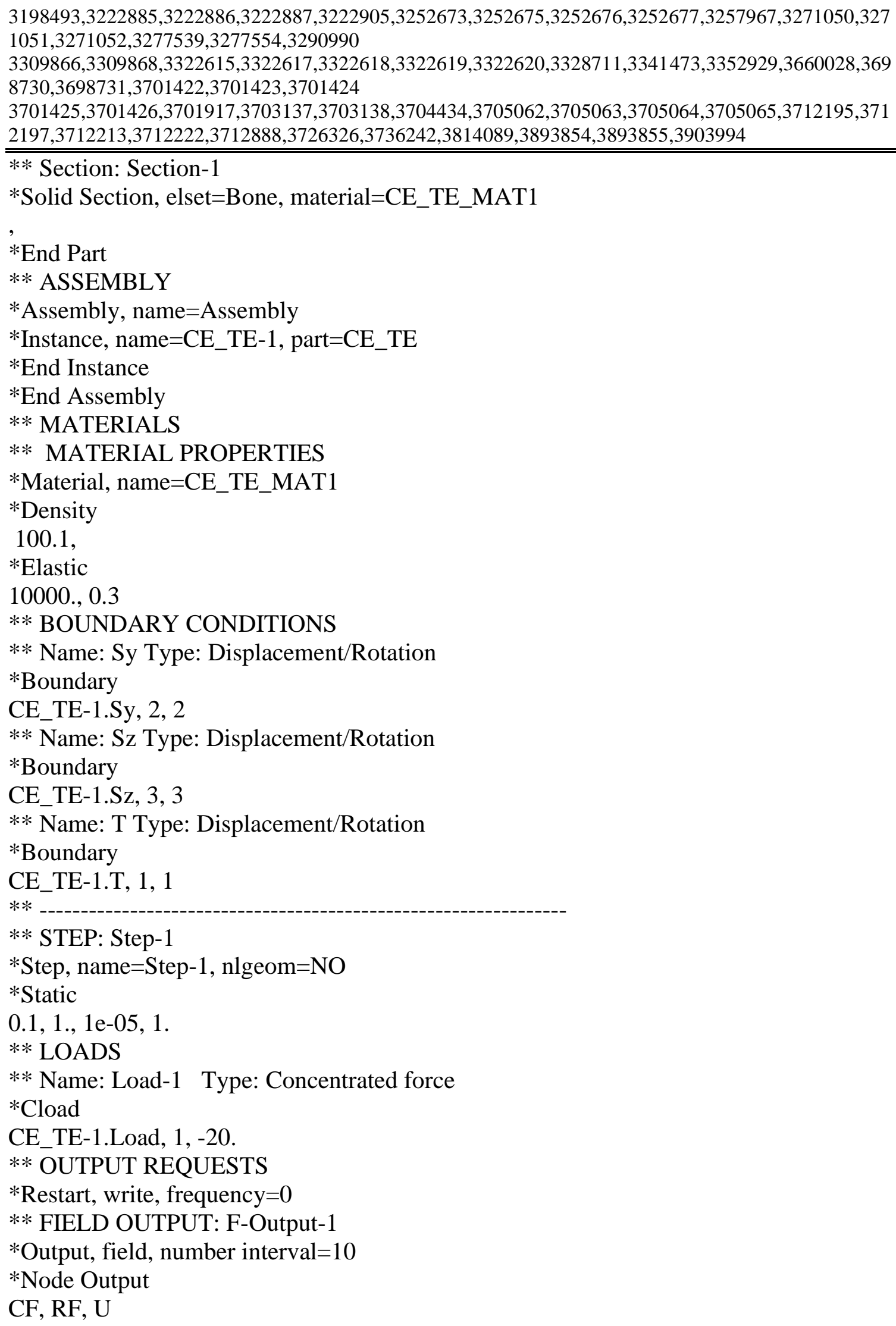


*Element Output, directions=YES

E, ELEDEN, EVOL, LE, MISES, MISESMAX, PE, PEEQ, PEMAG, S, STH, SVOL

*Contact Output

CDISP, CSTRESS, ENRRT

** HISTORY OUTPUT: H-Output-1

*Output, history, variable=PRESELECT, number interval $=10$

*End Step 


\section{Waleed Alim \\ -wmmmm_5@yahoo.com}

\section{PERSONAL STATEMENT}

A dedicated, detailed-oriented, and analytical research fellow. I have spent the past several years doing graduate-level image processing and bone biomechanics research. My project involved the development of a high-resolution finite element model of a human femur, the validation of that model through biomechanical testing of a fall-to-the-side hip fracture protocol, and parametric computational modeling of the effects of selective thickening and thinning of the cortical and cancellous bone tissue of the femoral neck. My results will provide guidance toward hip fracture prevention therapies in aging humans. I am a confident presenter at conferences, able to explain complex information to diverse audiences.

\section{EDUCATION}

\section{Ph.D., Mechanical Engineering, University of Louisville,} 2021

Concentrations: Bone Biomechanics

Dissertation: SIMULATION OF HIP FRACTURE STRENGTH IMPROVEMENTS

OF AN OSTEOPOROTIC PROXIMAL FEMUR USING FINITE ELEMENT

ANALYSIS

Dissertation Advisor: Michael Voor, Ph.D.

M.S., Industrial Engineering, Western Michigan University, 2009

B.S, Production Engineering, King Abdulaziz University, Saudi Arabia 1999

\section{RESEARCH EXPERIENCE}

\section{Computational modeling in four stages:}

- MIPAV \& Image-j software used for images processing including images cropping, noise removal, contrast enhancements, eliminating unconnected regions, image calculations, manual separation between cancellous and trabecular, and morphological processes.

- Mimics/3-Matic were used for thresholding, building 3D-surface, and remeshing optimization to eliminate potential errors in results like intersecting triangles.

- Finite Element Analysis was applied through Abaqus program in order to apply boundary and loading conditions that representing the conditions of the experiment.

\section{Experimental modeling:}


Exposing the bone to fracture test through soldering strain gauges at particular spots of the bone, connecting strain gauges with multimeter device, which is connected with a computer, and then orient the bone under a loading cell of the MTS machine to run the experiment.

\section{Validation results:}

The FE model is valid when the computational modeling strain is sufficiently close to the experimental measured strain at the same level of failure load as well as at the same gauges' location.

\section{PUBLICATIONS}

- Hip fracture strength due to fall-to-the-side loading: Relative effects of proximal femur bone quality and biomechanical properties (in progress)

\section{POSTERS}

- SIMULATION OF FRACTURE STRENGTH IMPROVEMENTS OF AN OSTEOPOROTIC PROXIMAL FEMUR USING FINITE ELEMENT ANALYSIS (FEA) (2020)

- Comparing two finite element meshing methodologies in high resolution simulation of proximal femur (2015)

- Cadaveric proof of concept testing of a novel exercise device for strengthening of the proximal femur to prevents hip fracture ( $2^{\text {nd }}$ author)

\section{RELEVANT SKILLS}

Two and Three Dimensional Image processing:

Micro-CT scanning, Image-j, MIPAV, Mimics, 3-Matic, VG StudioMAX

Finite Element Analysis:

ANSYS, ABAQUS, SolidWorks

Biomechanical Testing:

Materials Testing System (MTS Corp. Eden Prarie, MN), Strain Gauge analysis

Advance Engineering Math

\section{TEACHING EXPERIENCE}

Fluid Mechanics Lab

Fall

2016

Engineering Dynamics

Summer 2016

Math tutoring

Fall

2015

University of Louisville

Louisville, KY

\section{PROFESSIONAL MEMBERSHIPS:}

BMES 


\section{GOLDEN KEY INTERNATIONAL HONOR SOCIETY}

\section{WORK EXPERIENCE}

\section{Dissertation project}

- MIPAV \& image-j were used for images processing including cropping, noise removal, contrast enhancement, Masking, Boolean, conversion to binary, and morphology.

- Mimics/3-Matic were used for thresholding, region growing, creating 3D surface, meshing quality optimization, and then exporting input file for FEA.

- FEA was applied via Abaqus to simulate bone behaviors, and then validate results with experimental results

- MTS machine was used experimentally for applying boundary and loading conditions over the bone with strain gauges to obtain specific measurements.

\section{Student Assistant}

Sep/2015 - Apr/2016; Oct/2019 -

Aug/2020

KORNHAUSER Library - University of Louisville Louisville, KY

The position comprises multitasks and not limited to: Head counts, drop box, Newspapers, Desk, Scan/shelve, Inter-Loan Library, and Water bottles.

\section{Safety Engineer}

2000-2006

Saudi Company of Electricity

Arabia

- Implemented safety and security standards such as OSHA and NFPA

- Minimized working injuries more than 5\% annually by training $300+$ workers in electric power plant on basic personal protective equipment and occupational risks in plant

- Maximized the performance index $20 \%$ of my division Acting Supervisor of Industrial Security department $(2 / 03-5 / 06)$

- Strengthened the communications and improved the rate of responsiveness by arranging quarterly meetings between power plant administration and five governmental organizations for performing fire drills, emergency planning or safety issues

\section{LANGUAGES:}

Arabic Language (Native),

English language

\section{REFERENCES:}

Michael J. Voor, PhD

Dissertation Director, Department of Bioengineering

micheal.voor@louisville.edu 